\title{
BALANCING DATA, TIME, AND EXPECTATIONS: \\ THE COMPLEX DECISION-MAKING ENVIRONMENT \\ OF ENROLLMENT MANAGEMENT
}

\author{
By \\ ADAM W. JOHNSON \\ Bachelor of Arts in English \\ University of Central Oklahoma \\ Edmond, OK \\ 1995 \\ Master of Education in Adult Education - Community \\ Service \\ University of Central Oklahoma \\ Edmond, OK \\ 2005
}

Submitted to the Faculty of the

Graduate College of the

Oklahoma State University

in partial fulfillment of

the requirements for

the Degree of

DOCTOR OF EDUCATION

July, 2013 
BALANCING DATA, TIME, AND EXPECTATIONS:

THE COMPLEX DECISION-MAKING ENVIRONMENT

OF ENROLLMENT MANAGEMENT

Dissertation Approved:

Dr. Kerri Kearney

Dissertation Adviser

Dr. Ed Harris

Dr. Jesse Mendez

Dr. Lynna Ausburn 


\section{ACKNOWLEDGEMENTS}

Beginning this journey in 2008 , I really had no idea what to expect or what awaited me as part of this process. Realistically pursuing a dissertation had not entered my mind until I knew I had initial support from several people in my working life. My bosses during this experience, Dr. Myron Pope, Ms. Susan Wortham, and Mr. Jerry Legere helped encourage me from a professional standpoint while Mr. Allen Arnold, who had already begun his pursuit of a doctorate, always seemed to urge me to take the plunge as well. I truly feel blessed to have started coursework with several individuals in our Oklahoma City cohort who assisted, guided, counseled, and commiserated one another along the way and encouraged completion. Dr. Virginia Smith, Brad Williams, Torrie Campbell, Dr. John Fritch, LaKeisha Bryant, Josh Waddell, Nancy Spencer, Holly Kreidler among others - thank you for making our classes enjoyable, challenging, and worthwhile. Although not in my cohort, I also valued the feedback and guidance from Dr. Lisa Kerr who was able to offer a fresh perspective as well as astute observations of strengthening my dissertation.

I deeply appreciate my dissertation committee - Dr. Lynna Ausburn, Dr. Ed Harris, Dr. Jesse Mendez, and Dr. Kerri Kearney for their guidance, advice, and insight. Dr. Kearney deserves her own acknowledgement section as she has put up with my incessant questions, requests, and favors during this process but has been an unbelievable advocate (as she is to all her students) to ensure the dissertation gets finished!

Finally, and most importantly, I owe a tremendous thank you to my family. To my wife, Carolyn, and my sons Mason and Colin - you have demonstrated a level of

iii Acknowledgements reflect the views of the author and are not endorsed by committee members or Oklahoma State University. 
support and understanding for me during this entire process that I cannot fathom. There were so many nights where I turned to reading, studying, or writing instead of spending time with you. As prospective doctoral students, it was explained to us that family support was critical because there was no question you would be an integral part of our ability to finish coursework and defend a dissertation. I share this celebration with you and know it would not have been possible without your unwavering commitment and support. Although I am tremendously proud of what we have done, I also cannot wait to return to being a full-time dad and husband again. I love you all very much. members or Oklahoma State University. 
Name: ADAM W. JOHNSON

Date of Degree: JULY, 2013

Title of Study: BALANCING DATA, TIME, AND EXPECTATIONS: THE COMPLEX DECISION-MAKING ENVIRONMENT OF ENROLLMENT MANAGEMENT

\title{
Major Field: HIGHER EDUCATION
}

\begin{abstract}
:
As a growing entity within higher education organizational structures, enrollment managers (EMs) are primarily tasked with projecting, recruiting, and retaining the student population of their campuses. Enrollment managers are expected by institutional presidents as well as through industry standards to make data-driven planning decisions to reach their goals. However, despite the availability of data, some enrollment managers revealed a different reality from traditional, rational decision-making models. This study explored the factors that contributed to or influenced the decision-making process of six enrollment managers working in either two or four-year public colleges and universities located in the Midwestern United States. Interviews, including a word association exercise, documents and artifacts of tools utilized to make enrollment management decisions, and observational field notes were gathered and analyzed in this qualitative study grounded in constructionism. Using the theories of Bounded Rationality and the Garbage Can model as lenses, EMs in this study seemed to reflect a highly stressful, competitive, and complex environment where rational decision-making gave way to satisficing, or "good-enough" decisions as expectations to meet enrollment goals governed their motives. Decisions also appeared to be closely associated to the Garbage Can Model due to the ambiguity of goal setting and the mixture of participants, problems, and solutions where temporal proximity appeared to be more of a factor in terms of what received attention first. With multiple interruptions into an EM's daily schedule, the ability for the enrollment manager to maintain consistent efforts on desired projects and plans was severely hindered. Enrollment managers in this study shared through multiple examples that they did not feel they had enough time to accomplish their tasks as thoroughly as they preferred and often addressed their problems without full consideration of all possible options, much less the optimal one.
\end{abstract}




\section{TABLE OF CONTENTS}

$\begin{array}{ll}\text { Chapter } & \text { Page }\end{array}$

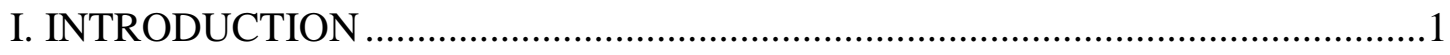

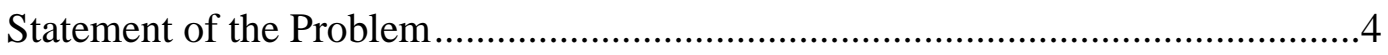

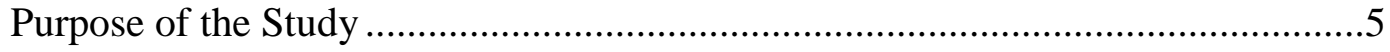

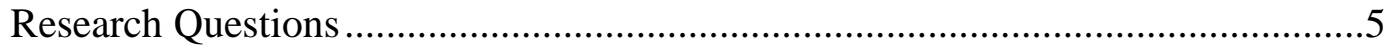

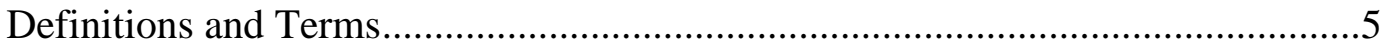

Orienting Theoretical Framework.................................................................6

Bounded Rationality and Satisficing ……………........................................

The Garbage Can Model ...............................................................................

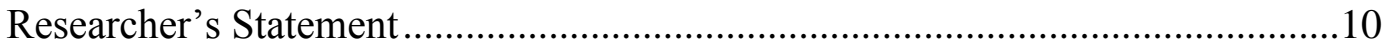

Data Collection Procedures and Methods ............................................................

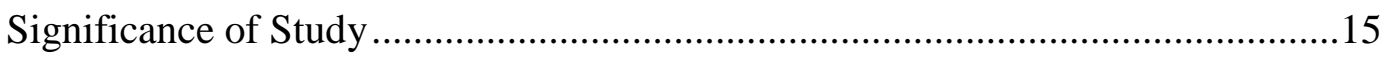

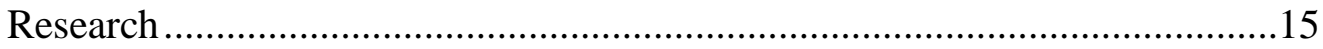

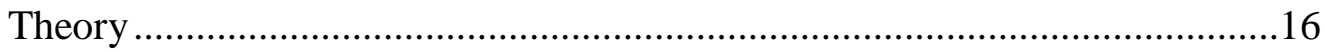

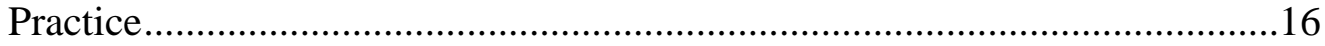

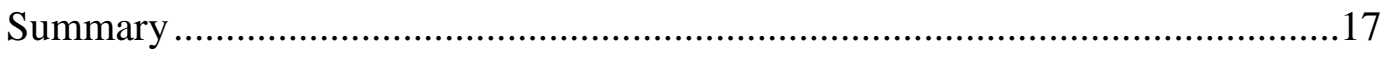

II. A REVIEW OF EXISTING LITERATURE ...................................................19

Enrollment Management Overview ................................................................21

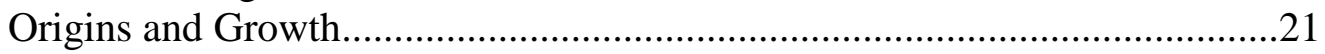

Enrollment Management Models.....................................................................2

The Enrollment Management Committee........................................................23

Enrollment Management Coordinator/Director ................................................24

Enrollment Matrix System ........................................................................2

Enrollment Management Division ...............................................................25

Strategic Enrollment Management ..............................................................25

Expectations and Characteristics for Enrollment Management Leaders .........26

Role of Data/Research in Enrollment Management ......................................28

Professional Associations and Consulting Firms ................................................32

Future of Enrollment Management ...................................................................34

Organizational Theory in an Enrollment Management Context..............................35

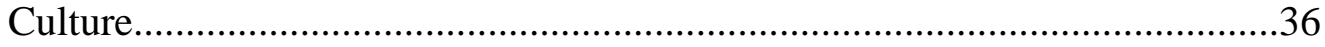

Governance and Leadership............................................................................

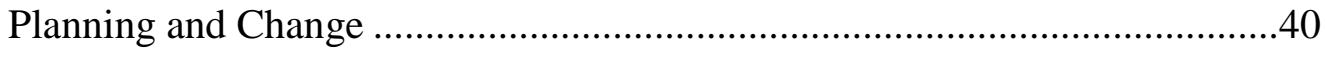

As an Open System ................................................................................... 41 


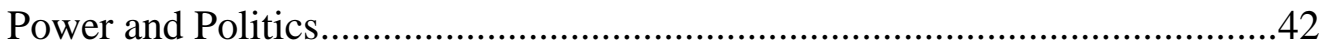

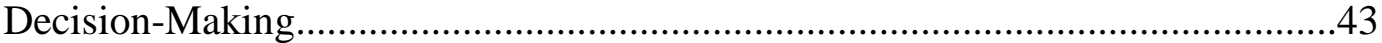

Organizations and Decision-Making .............................................................

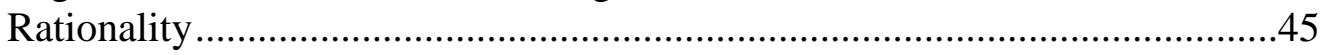

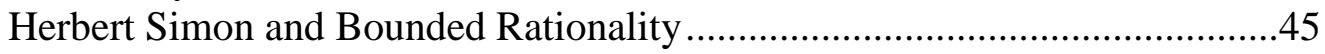

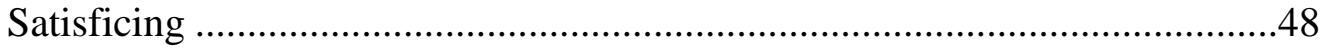

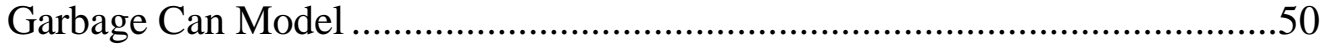

Pairing Satisficing and the Garbage Can Model to Enrollment Management.52

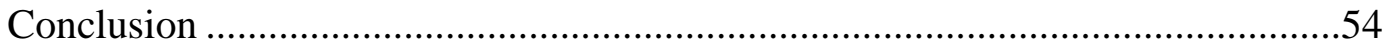

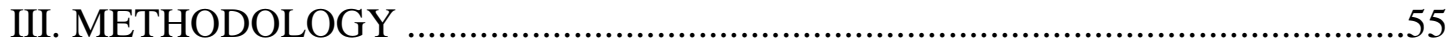

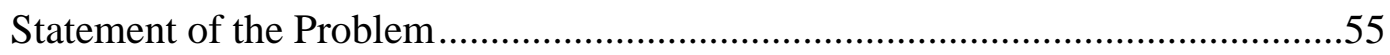

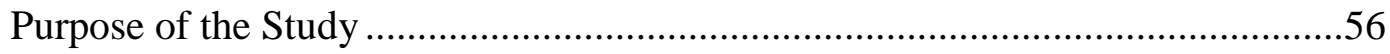

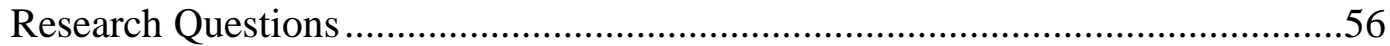

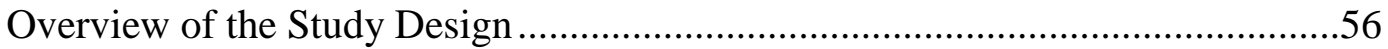

Guiding Epistemology for This Study ...........................................................57

Influences on the Study and Theoretical Framework .........................................59

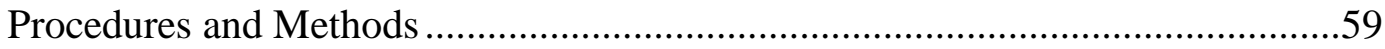

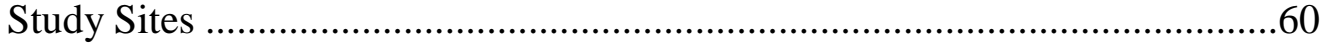

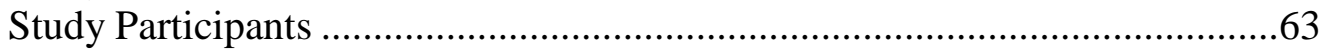

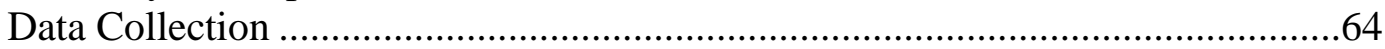

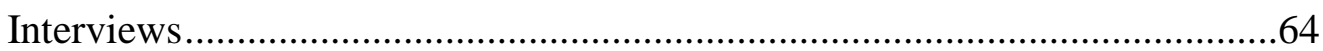

Documents and Artifacts.........................................................................64

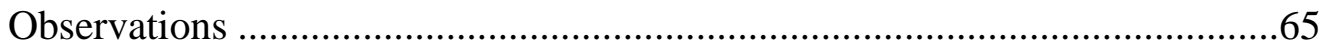

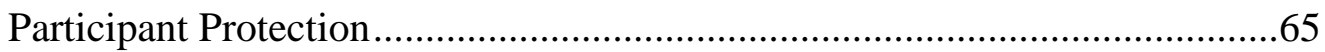

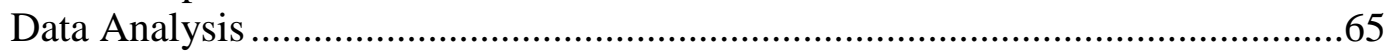

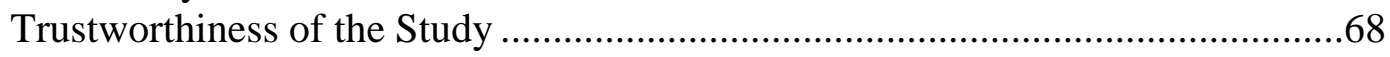

Implications for Research, Theory, and Practice ...............................................69

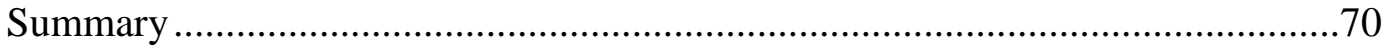

IV. DESCRIPTION OF SITES AND PARTICIPANTS ............................................72

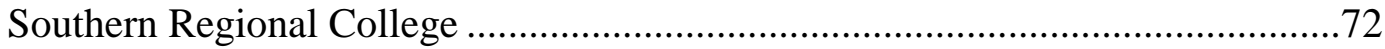

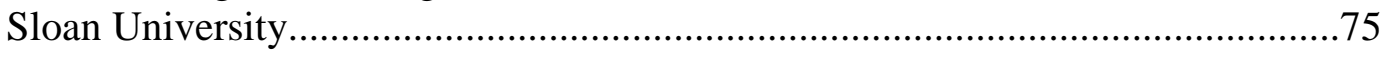

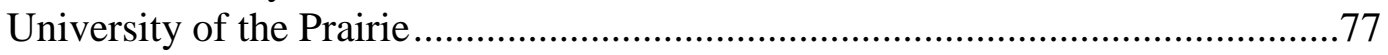

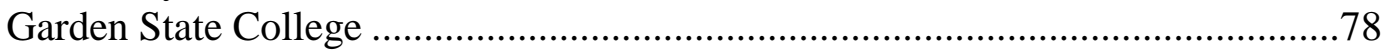

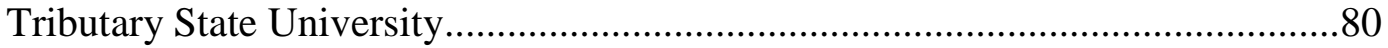

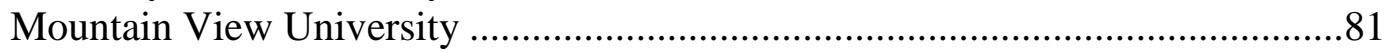

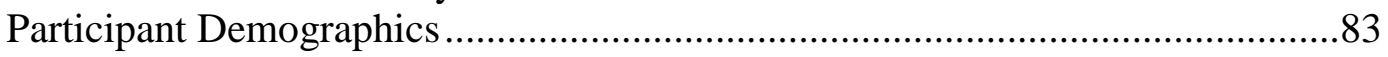

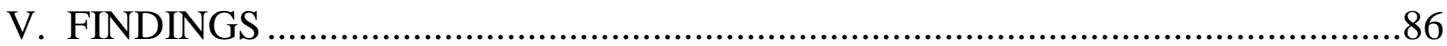




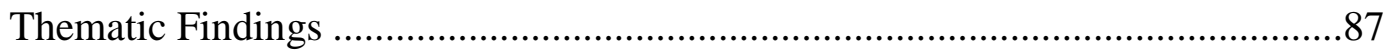

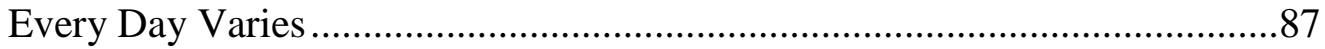

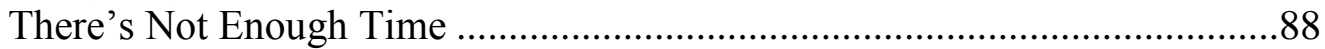

Enrollment Management is a Competitive Environment....................................89

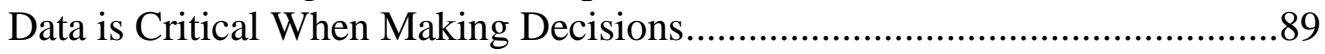

Good, Usable Data Can Be Difficult to Get ...................................................90

Sometimes, You make Decisions on Instinct and Experience..........................91

Reactive vs. Proactive Decision-Making ………………............................91

We Must Have Strategic Planning ..................................................................92

"Everything Comes Back to the Numbers" ...................................................92

"Who's Screaming the Loudest" ................................................................93

Consultants Aren't the Answer ...................................................................94

Faculty Need to Be Our Partners ....................................................................95

Enrollment Expectations Aren't Always Realistic .........................................95

We Have Good Employees, But There Are Not Enough of Them...................96

Stable Leadership is Essential.....................................................................97

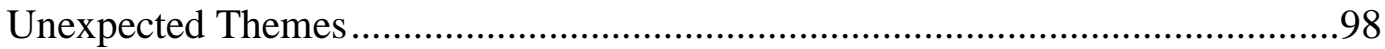

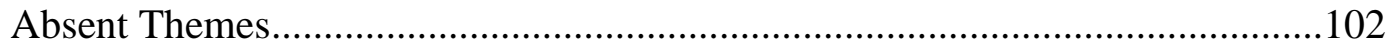

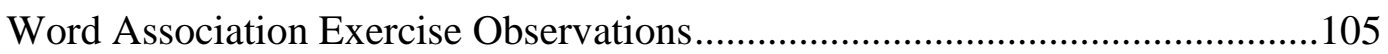

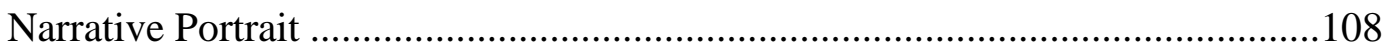

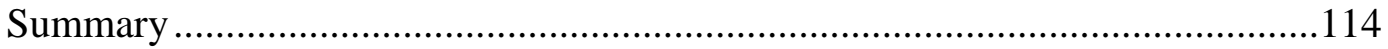

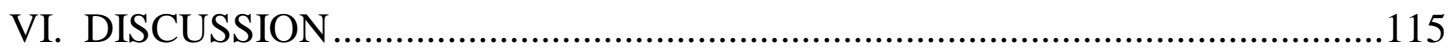

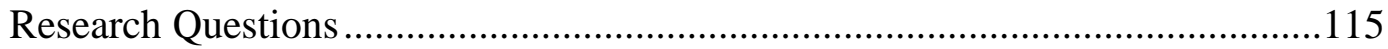

Summary of Participant Descriptions and Findings ..........................................116

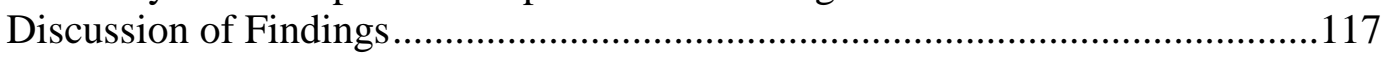

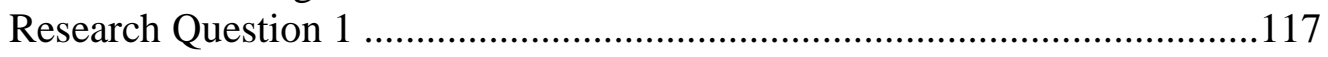

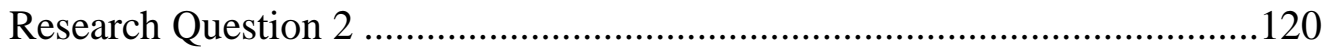

Research Question 3 …………………................................................122

Themes Through Theory ............................................................................123

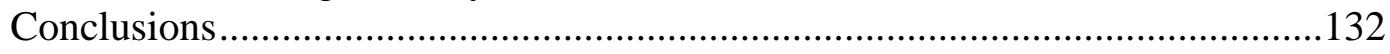

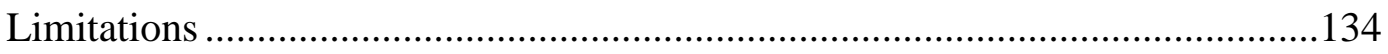

Implications for Research, Theory, and Practice ..............................................135

Recommendations for Future Research ...........................................................140

Final Summary and Reflection ....................................................................143

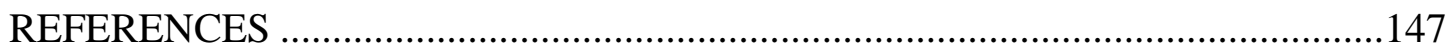

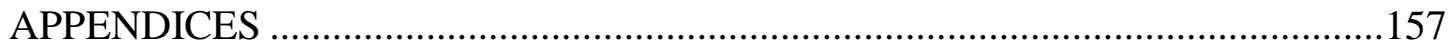

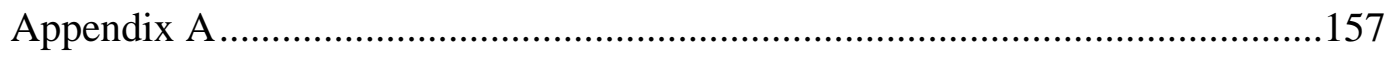

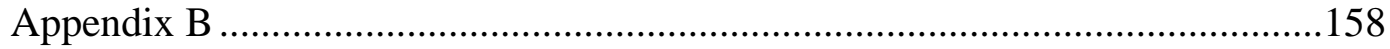

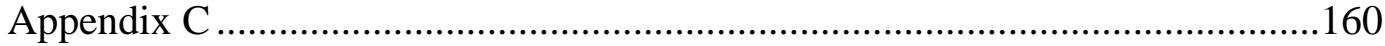

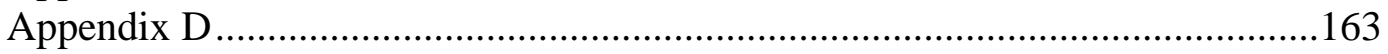


Appendix E

170

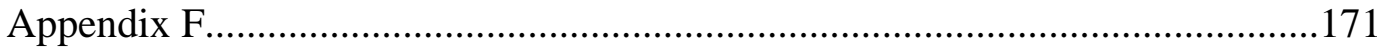




\section{LIST OF TABLES}

Table Page

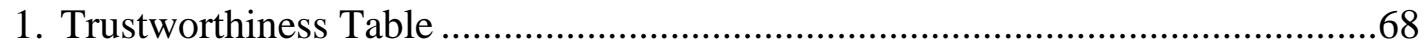

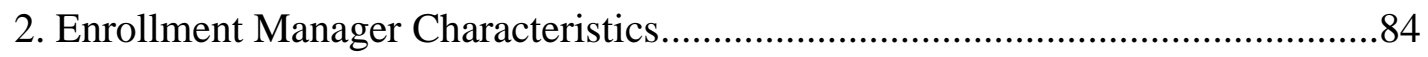

3. Word Association Exercise and Responses .............................................. 105 


\section{CHAPTER I}

\section{INTRODUCTION}

Once an emerging profession in the 1970's, the organizational concept known as enrollment management has evolved into an administrative function tasked with the responsibility of strategically recruiting and retaining students. The divisional model is the most prevalent, but some campuses utilize institution-wide teams (Hossler, 1984; Penn, 1999). Regardless of format, the number of institutions utilizing the practice of enrollment management has grown from a small handful of private institutions to widespread among most public and private institutions (Hossler, 2004).

The enrollment management approach initially began as a reaction to address fluctuating enrollment patterns by instituting more purposeful and collaborative recruitment efforts along with financial aid leveraging to help attract students (Hossler, 1986; Machado-Taylor, Peterson, Taylor, \& Wilkinson, 2007). However, a true consensus of what defines enrollment management does not exist as a variety of sources have tried to classify or demarcate the necessary components of the enrollment management concept (Dennis, 1998). Baldridge, Green, and Kemerer (1984) offered a description of enrollment management as both a "concept" as well as a "procedure," (p. 21) while acknowledging the complexities of not only encapsulating an enrollment management definition but also the task of predicting or maintaining college enrollments. 
Descriptions and applications of enrollment management approaches remain fluid, but this is also somewhat intentional. Enrollment managers incorporate campus-wide coordination to identify institutional enrollment goals, but these collaborative endeavors must adapt to a fluctuating educational system that treats students as customers who know the value of their educational experience. Developing marketing/recruitment plans, registration procedures, retention strategies, and financial aid packaging is a crucial component of enrollment management, but this must also be balanced against the academic and political environment of the institution (Dennis, 1998). While an agreed-upon definition may not be universally accepted, all enrollment management leaders should expect to make prudent yet calculating planning decisions to reach their enrollment goals despite the complexity of this environment (Hossler, 1986). For the purposes of this study, enrollment manager (EMs) will be defined later in this chapter under the Definition and Terms section.

The field of enrollment management has always advocated for strategic planning incorporating multiple areas and individuals across a campus to develop a cohesive enrollment plan according to its mission. Designing a marketing or recruitment strategy to effectively represent a college or university should occur after decisions have been made concerning the preferred characteristics of the desired student. Regardless of the results of the marketing efforts, the strategies to attract the ideal student as well as the plans to retain them must be evaluated (Hossler, 1984). Additionally, enrollment management practitioners are expected to be able to effectively forecast enrollment trends or patterns while balancing controllable and uncontrollable variables. In other words, EMs can specifically target the desired population of students desired for their institution, but they are unable to control factors such as high school graduation rates. Although numerous computer programs and 
software exist to help aid in this planning process, there is no replacing the role of the human administrator to make the decisions (Baldridge, Green, \& Kemerer, 1982).

Despite the obvious need and demand for strategic planning, certain barriers exist which inhibit its success. Implementing a strategy or initiating a change process has proven to be a slow practice in higher education. Stagnant or dwindling budgets coupled with the shared governance required of enrollment management structures contribute to a sluggish movement toward progress. Also, any type of plan should not be copied verbatim from another institution but instead be tailored to the individual college or university in order to be truly connected to its mission and overarching the institutional strategic plan (MachadoTaylor, Peterson, Taylor, and Wilkinson, 2007).

Successful planning is contingent to the availability of data to inform enrollment management practitioners when making decisions. Dennis (1998) suggests a minimum of fifteen routine reports that should be made available to decision makers. These include enrollment for each student cohort, financial aid impact upon specific cohorts of students, the influence of advertising and publications on enrollment, analysis of enrollment for new markets, and others. A listing of over seventy professional and specialized publications is also recommended to be read quarterly, monthly, and even weekly. However, all of these tools could be rendered worthless if presented in an unusable manner (Baldridge, Green, \& Kemerer, 1982).

Riley (2007), in his dissertation, suggested that administrative leaders, especially those in an enrollment management leadership role, have tremendous difficulty making effective decisions despite the availability and access to information and data as expressed above. Considering the complexity of the enrollment management environment, one which 
requires campus-wide communication and involvement, the possibility of fragmented information and inadequate preparation for effective decision-making exists. Both problems and solutions can become ambiguous as enrollment management leaders, who may have been placed into key decision-making roles without the proper background or experience in planning and implementation, become buried under the complexity of data elements which leads to an uncertainty of action.

There is an urgent need to understand and learn about these intricate environments, especially as it pertains to the process enrollment management practitioners use to make decisions. As institutions face budget shortcomings coupled with increased expectations to reach enrollment goals, learning about the methods and strategies informing the decisionmaking of the EM becomes increasingly important.

\section{Statement of the Problem}

As a growing entity within higher education organizational structures, enrollment managers (EMs) are primarily tasked with projecting, recruiting, and retaining the student population of their campuses. These enrollment managers are expected by institutional presidents as well as through industry standards to make data-driven planning decisions to reach their goals (Baldridge, Green, \& Kemerer, 1982).

However, despite the availability of data, some enrollment managers revealed a different reality from traditional, rational decision-making models. Their experiences reflected incomplete, fragmented, and ambiguous solutions that impacted the overall quality and efficiency of decision-making within the enrollment management areas (Riley, 2007).

Considering that EMs are expected to incorporate a campus-wide and collaborative effort in their plans, as well as the vast amounts of data in the decision-making process, this 
incongruence may exist due to complex factors within the enrollment management environment that may inhibit rational decision-making.

\section{Purpose of the Study}

The purpose of this study was to explore the factors that may contribute to or influence the decision-making process of enrollment managers working in two and four-year public colleges and universities located in the Midwestern United States; the study also looked specifically at how EMs use available data. Considering not every higher education institution contains bona fide enrollment management units, divisions, or teams, only those institutions who utilize those formal functions were explored in this inquiry.

\section{Research Questions}

1. How are strategic enrollment management decisions made on a campus that utilizes an enrollment management system?

2. As enrollment management professionals make decisions, what do they consider?

3. How do expectations relate to decision-making?

\section{Definitions and Terms}

Data Mining - "It is a process of problem identification, data gathering and manipulation, statistical/prediction modeling, and output display leading to deployment or decisionmaking” (Schumann \& Streifer, 2005, p. 283).

Enrollment Management - The holistic, intentional efforts for institutions to implement assertive strategies to ensure a consistent stream of desired, qualified enrollees to preserve an institution's livelihood (Baldridge, Green, and Kemerer's, 1982). 
Enrollment Manager (EM) or Managers (EMs) - The individual who is primarily responsible for maintaining and managing enrollments which may include responsibilities associated with retention and graduation.

Strategic Enrollment Management (SEM) - "SEM practices incorporate the institution's overall mission while adhering to the principles of establishing clear enrollment goals; promoting student success; determining, achieving and maintaining optimum enrollment; enabling the delivery of effective academic programs; generating tuition; enabling financial planning; increasing organizational efficiency; and improving service levels" (MachadoTaylor, Peterson, Taylor, \& Wilkinson, 2007, p. 6-7).

Yield - The conversion percentage of applicants to admitted students, admitted students to enrollees, or applicants to enrollees.

\section{Orienting Theoretical Framework}

Before presenting details concerning the collection of data and associated procedures, it is important to communicate the researcher's philosophical epistemology or worldview and its relevance to this study. Creswell uses the term "worldview" (p. 6) to articulate how one generally views the world in a research-based context. Crotty (2003) utilizes the term "epistemology" and explains it as demonstrating how one looks and understands the surrounding world, or, "how we know what we know" (p. 8).

The guiding epistemology for this particular study was constructionism (sometimes also referred to as social constructionism). Contrary to an objectivist viewpoint, which asserts that meaning exists without engagement with any external source, those who possess a constructionist epistemology posit that knowledge and meaning is constructed by the interaction and engagement of humans upon objects in the world (Crotty, 2003). Reality, 
meaning, and knowledge are therefore constructed by the individual and applied to an object or situation in one's life (Patton, 2002).

Crotty (2003) expands upon the constructionist view through his description of social constructionism, or more specifically, the impact of one's culture upon making meaning of experiences. Culture is seen as "the source rather than the result of human thought" (p. 53). Humans apply meaning through a social context, and all experiences are deemed as meaningful realities in social constructionism. One's behavior and evaluation of experiences can be traced back to cultural influences, and these influences provide the lens for assessing, valuing, and applying meaning to each context.

In its truest form, constructionism recognizes that there is no single reality. Multiple realities not only exist, the same situation or occurrence can mean a tremendous amount of diverse interpretations and understandings (Crotty, 2003). Considering the varied realities experienced by assorted enrollment managers at their respective institutions, this epistemology reflected the range of interpretations upon their decision-making realities.

This research study was one of exploration and emersion into the experiences of those working in areas within enrollment management. The type of method best suited for this form of research was a qualitative inquiry. As it is the intent of the researcher to be able to share the experiences and perceptions of the study's participants, a qualitative inquiry allows for thick, rich details which help reveal their realities (Patton, 2002).

Although my own experiences concerning decision-making in an enrollment management context greatly influenced the overall construction of this inquiry, this study was also influenced by perspectives and suggestions found in Riley's (2007) dissertation of a similar topic. While his quantitative study measured the decision-making effectiveness of a 
particular institution's enrollment managers, I chose to conduct a qualitative study to reveal the decision-making experiences of enrollment managers in multiple institutions and contexts.

This study also utilized the theoretical framework outlined in Riley's study - Herbert Simon's model of bounded rationality and satisficing as the lens to help illuminate the emerging results from the study (Lehman, Lyubomirsky, Monterosso, Schwartz, White, \& Ward, 2002). While Simon's bounded rationality theory helped illuminate how decisions are made by the individual, March, Cohen, and Olsen's garbage can model also served as an additional perspective for the types of complex and inter-related decisions made by enrollment management entities (Cohen, March, \& Olsen, 1972; Lane, 1980). The applicability and usefulness of each theory was determined and discussed after data collection and analysis.

\section{Bounded rationality and satisficing.}

Herbert Simon became a staunch critic of the pure rational choice models when applied to the nature of humans who do not always operate from a logical and linear approach toward making decisions. Simon argued that realistically, humans do not possess full access to the complexity of the environmental factors nor do they have a fully effective cognitive system that can maximize every real-life decision (Campitelli \& Gobet, 2010).

The theory of bounded rationality therefore suggests that individuals can and will make decisions with practical and available calculated factors, and this information will be incomplete in scope. Nevertheless, despite not having a fully stocked cache of data or information available at the time a decision is desired or required, reasonably solid decisions will be made. Furthermore, it is unrealistic to expect that humans have all possible 
alternatives calculated and evaluated for the purpose of selecting the most effective option (Campitelli \& Gobet, 2010).

Simon suggested the additional concept of satisficing nearly a half-century ago and introduced it as a means of more accurately and realistically explaining how humans make decisions. His goal of maximizing choices to find an exacting and optimal solution was overwhelming unrealistic. Therefore, he claimed humans will actually look for a solution that is "good enough," and that there is a great deal of variance in the levels of how that choice meets the expectations. The point is that it is met-even though it is often not the absolute best choice. Satisficers are not driven by the goal to discover the maximized and optimal solution but merely an adequate one (Lehman, Lyubomirsky, Monterosso, Schwartz, White, \& Ward, 2002, p. 1178).

The garbage can model

Despite the insinuation that garbage can implies discarded ideas, this model actually reflects a mixture between problems, solutions, participants, and opportunities. This model has often been used to describe higher education organizational structures (Cohen, March, \& Olsen, 1972; Lane, 1980). The model suggests organizations function in a manner of organized anarchy which allows for a great deal of ambiguity when attempting to define organizational goals as well as determining who is going to accomplish them. Utilizing this theory may help to understand the collective combination and mixture of problems, solutions, participants and opportunities in addition to the dynamics and complexities surrounding them (Padgett, 1980).

The two models balanced and complemented each other well as they both reflected the challenges individuals (and their organizations) faced in terms of meeting all the demands 
and expectations for a decision. An individual may feel compelled to make a satisfactory decision as opposed to a maximized one, and this seemed to be a very appropriate reality for some higher education systems (Lutz, 1982).

Each of the models was applied a priori in the design due to its applicability in understanding the decision-making processes of enrollment managers. Initially, they were held in queue contingent upon data analysis, and both reflected greater meaning by using these models as appropriate lenses. Riley's (2007) dissertation utilized these models in his

study, and I considered viewing the data gathered through the perspective of these models as the evidence reflected substantiated meaning.

\section{Researcher's Statement}

In order to offer the reader an appropriate insight into my motivation and interest in this study, the following section will present information concerning my career interests, educational background, life experiences, and personal encounters that may serve as a filter for the collection as well as analysis of the data.

Although I did not begin my professional career working in higher education, I recognized my passion and enthusiasm for it when I began working as a recruiter for a large, 4-year public institution in Oklahoma (my alma mater) nearly fifteen years ago. I worked in the Prospective Student Services/Scholarships (PSS/S) office, and my assigned recruiting territory was the northwest region of Oklahoma. At that time, I was unaware of a model known as Enrollment Management, but after witnessing the campus-wide attention our office received given the expectations and responsibilities for increasing enrollment goals, I can now fully understand upon reflection how our office (and others) would become part of an entire division focused on the management of student enrollment. 
After successfully meeting my recruiting objectives for nearly three years, I sought a higher-level position at a larger, 4-year state institution and was hired to target high school students in the Oklahoma City metropolitan area. This institution's recruitment office was called High School and College Relations (HSCR), but its function was quite similar to the PSS/S office. Once again, however, there was no mention of either a formal or informal concept of Enrollment Management. A more nationally recognized university, this campus felt even stronger pressure to meet institutionally-established enrollment goals.

In this position, I witnessed a different process for decision-making than the previous institution. At the former university, decisions were made in a team-based environment where comments made by each team member appeared to be valued. The use of a variety of data sources was limited, but the recruitment office relied on what data existed. There was recognition, however, that key data elements or even the ability to gather such data was missing.

At the new institution, a more authoritative presence was felt in terms of the decisionmaking process, as well as a stronger influence by upper administrators to direct those choices. This campus possessed more resources (both human and financial) as data sources were more obtainable and accessible; however, decisions seemed to be made arbitrarily at times as if anecdotal information was appreciated more by the decision-makers than computer-driven reports.

It was not until I returned to my alma mater (to work in an administrative position) that I began to hear rumblings and rumors of the creation of a new division tasked with enrollment management. Rather than focus and devote resources toward attracting incoming students, a growing concern was expressed to begin addressing institutional issues associated 
with poor retention and graduation rates of students. Due to both internal and external influences, these concerns swelled to the point that action was soon required.

Two years after I returned to my alma mater, our office of PSS/S merged with Admissions to become the Office of Undergraduate Admissions. More importantly, this new office was to be part of the newly formed Division of Enrollment Management. Additional offices, which previously belonged in the divisions of Academic Affairs or Student Affairs, were acquired, and those included Academic Advisement, Student Financial Aid, and the Bursar. Two new offices, the Office of First Year Experience and the Transfer Center were also created and placed in this division.

The new Vice President for Enrollment Management hired me as Director for Recruitment and Scholarships in Undergraduate Admissions, and he introduced me to the concepts and strategies associated with Enrollment Management. However, I also recognized the complexity of collaboration and coordination required to become an effective enrollment management division, especially as it applied to making sound data-driven decisions. I felt a substantial amount of personnel was handicapped by limited knowledge both conceptually and through applicability of enrollment management concepts. In other words, we were a division in name only; few understood the implications or responsibilities associated with being a part of this new division.

To further my understanding and development in recruitment and admissions-related practices, I attended conferences specifically based on Strategic Enrollment Management (SEM) principles sponsored by the American Association of Collegiate Registrars and Admission Officers (AACRAO). I distinctly remember one presenter offering this axiom as it applied to data gathering and analysis: "If it moves, track it." This left an indelible 
impression upon me as an enrollment management practitioner. I felt that not only should our institution amplify existing efforts to track recruitment, retention, and graduation strategies' efforts, but that decisions were going to be more dependent upon on the amount and quality of all of this gathered data.

With my current role as an Associate Vice President/Registrar within the same institution and division, the need for data gathering and analysis has intensified. Through discussions with colleagues at other colleges and universities (as well as individuals holding management positions at my own institution), it appears that we are all facing similar challenges when it comes to creating reporting strategies to "track" efforts and to take action necessary to address or implement them. Despite possessing the desire to use data, many of us encounter difficulty in utilizing (and in many cases, gathering) the information to make sound, comprehensive, and rational decisions.

My own experiences combined with observations and conversations with EMs concerning the complexity of data used when making decisions spawned my interest in developing a qualitative study to investigate these issues. I discovered Simon's model of bounded rationality while researching decision-making strategies and models, and Riley's (2007) dissertation provided an enrollment management context that utilized both the bounded rationality/satisficing as well as the garbage can model for decision-making. While Riley's study examined the effect of a particular decision made at a single institution, I preferred investigating the decision-making experiences of EMs located at various institutions to present a broader perspective.

My career experiences, including serving as an active member of OACRAO, provided interaction with multiple colleagues from institutions across the state of Oklahoma. 
Therefore, I was familiar with some of the prospective participants as well as their enrollment management organizational structure. Although not discussed in terms of a suggested study, the topic of using data to drive decisions had been discussed at various OACRAO-based events as well as during informal encounters.

\section{Data Collection Procedures and Methods}

Consistent with qualitative methodology, I served as the instrument when gathering and interpreting data. To ensure validity, I endeavored to optimize the skill and competence necessitated for credibility of the study's results. A variety of methods were employed to supply thick, rich data that will minimize researcher bias and subjectivity (Patton, 2002). I also developed and utilized some of the writing strategies presented by Wolcott (2009) including freewriting, obtaining feedback, and coding.

I conducted one-on-one interviews containing open-ended questions (probing as necessary), explored documents and artifacts including recruitment plans, enrollment and retention reports, organizational charts, mission and vision statements, and other tools employed to make decisions. In addition, I gathered observational field notes derived from site visits and investigated tools related to each EM's decision-making for the respective institutions. The purpose for the varied methods of collecting, observing, presenting, and analyzing data was to develop triangulation, which Patton (2002) indicated would help strengthen a study.

Upon completion of data gathering, an analysis of the interviews, field observations, and documents and artifacts was commenced to assist with interpreting and coding the data (Wolcott, 2009). Emergent themes from the various data sources were identified through what Emerson, Fretz, and Shaw described as pursuing members' meanings when coding the 
data (1995). Assisting with the coding analysis and theme identification were the use of analytic statements which helped connecting thematic elements with initial insights and fieldnotes to help answer, "What's going on with the data? (p. 146, 1995)."

Six institutions situated in the Midwest were selected for participation. This list was established based on purposive sampling strategies designed to reflect 2-year, 4-year, public and private college and university enrollment management contexts (Patton, 2002). More detailed descriptions of the guiding selection criteria and each institution can be found in Chapter Three.

Methods of interpreting data included jottings and detailed notes which combine to capture an accurate and natural representation of the participant's environmental surroundings (Emerson, Fretz, \& Shaw, 1995). Data was collected and maintained in a secure location at my home to prevent tampering with or altering the process. In order to demonstrate validity of the analyzed results, a trustworthiness table was supplied (see Chapter Three). Application of this table helped ensure credibility and dependability associated with the findings in the study as well as achieve appropriate evaluative credentials (Guba \& Lincoln, 1985).

\section{Significance of Study}

\section{Research}

This qualitative study investigating the decision-making practice of enrollment managers assisted in filling in gaps of research available on the topic. Although many sources offer descriptions and strategies associated with enrollment management as well as decision-making approaches, few studies bring them both together, especially in a qualitative 
design. This study brought clarity to the decision-making realities experienced by EMs while trying to satisfy institutional enrollment expectations.

\section{Theory}

The research gathered in this study helped demonstrate further relevance of organizational decision-making theories such as Simon's bounded rationality theory as well as March, Cohen, and Olsen's garbage can model and their applicability in enrollment management settings. Should organizations attempt to craft or mold their structure to adhere toward a specific decision-making method or model, the evidence put forth in this study may help enrollment management practitioners plan and adapt accordingly.

\section{Practice}

Despite the numerous tools available to assist enrollment planners in their recruitment and retention of students as well as the increased attention given to the practice of enrollment management by professional organizations and assorted affiliations, managing an institution's enrollment remains a complicated task. If it weren't, computer models would simply project target markets necessary for maximization for enrollment. The complexity of this environment necessitates direct human involvement to discriminate the key factors from nebulous ones, and this subjectivity and individuality must be intertwined with enrollment management decision-making.

As state legislatures and governing boards continually decrease funding to state institutions and private institutions face economic challenges due to a weak economy, the decisions enrollment management leaders make may significantly impact their institution's goals. Gaining a greater understanding of how the decision-making progresses, and 
especially how well expectations match reality, will help improve EMs' ability to plan, improve, or modify existing decision-making practices.

\section{Summary}

Enrollment managers are tasked with projecting, recruiting, and retaining the student populations on their campus. Inclusive of this expectation is the reliance on ample amounts of data to help make informed and data-driven decisions to reach institutional enrollment goals (Baldridge, Green, \& Kemerer, 1982). The experiences of some enrollment managers reflected a different reality from the traditional rational decision model. Interviews, documents, and artifacts, collected from enrollment managers employed at two and four-year public and private institutions in the Midwest, helped illuminate their experiences with decision-making.

It is my hope that the results of this study will assist enrollment managers as well as institutional leaders to understand the decision-making processes experienced by these participants. Considering the reliance on these individuals to produce and manage enrollment goals necessitated by institutional presidents and governing bodies, as well as the financial dependence on these students, the significance of decision-making effectiveness becomes clear.

Chapter Two presents pertinent information concerning the purpose and need for enrollment management, organizational theory in an enrollment management context, and a description and applicability of bounded rationality decision-making strategies. Chapter Three describes the methodology and research design followed by Chapter Four which presents information concerning site visit observations and participant characteristics. The fifth chapter explores the themes revealed from the data. Chapter Six offers answers to the 
research questions, discusses the findings using theory as a lens, makes recommendations for further research into the topic, and presents final conclusions. 


\section{CHAPTER II}

\section{A REVIEW OF EXISTING LITERATURE}

Whether a formally recognized enrollment management structure exists in the form of a committee, coordinator/director, combined matrix of decision makers, or division, enrollment management practitioners and advocates assert the need for coordinated collaboration to reach enrollment goals (Baldridge, Green, \& Kemerer; Dennis, 1998; Hossler, 1984; Hossler, 1986; Machado-Taylor, et al, 2007). While definitions and application of enrollment management models have expanded beyond the initial descriptions and principles brought forth in the 1970s and 80s, the need for managing college enrollment remains. Reductions in state appropriations and foundation dollars remain at the forefront in terms of institutional financial concerns, but fluctuating enrollment patterns linger just behind as institutional planners evaluate the key factors keeping their institutional financially viable (Dixon, 1995b; Penn, 1999).

It is expected that enrollment managers will use data for all enrollment management planning efforts. However, this expectation asserts that the planning initiatives should be grounded in reality. The evidence and data, when evaluated, should help equip enrollment management practitioners to recognize when to take action as well as when to exert restraint. For example, if an institutional value or goal is quality or diversity of applicants, attempts to increase overall enrollment numbers 
should not come at the expense of the desired diversity. Data will help ensure that institutional aspirations are met (Black, 2004).

Just as enrollment management models vary across institutions of higher learning, what EMs consider valuable and useful data to assist in the decision-making process differs as well (Salomonson, 2005). Enrollment management practitioners have deemed timely and accurate information as paramount in their ability to effectively manage and shape enrollment. Despite this repeatedly stated need for accurate information, problems with organizing large quantities of data have been common for over two decades (Clagett, 1991). Several sources have labeled the process for collecting, monitoring, or tracking data while adhering to the institutional and academic mission as complex (Black, 2004; Clagett, 1991; Riley, 2007; Salomonson, 2005). With the ever-evolving and moving target of institutional goals (Black, 2004), a lack of information exists as to how EMs are expected to adjust and adhere to the strict expectation of making data-driven decisions.

Three major areas will be presented in this literature review to allow the reader a more detailed background into the issue as well as help substantiate the need for investigation of the problem. The first area delves into topics concerning the origins and ongoing evolution of enrollment management and its leaders. Second, an examination of enrollment management as an organization including the roles culture, governance, and other factors that impact the organization will be presented. Finally, further explanation of decision-making strategies expected to be utilized by EMs will be explored. In combination, these sections will showcase a complex environment with elevated expectations placed on its leaders who are asked to make precise and well-researched 
decisions that will directly impact and direct the financial and academic course of the institution.

\section{Enrollment Management Overview}

\section{Origins and Growth}

Although some sources offer different definitions of the term enrollment management, Baldridge, Green, and Kemerer's 1982 book Strategies for Effective Enrollment Management offered an early encapsulation of its intent, which is for institutions to implement assertive strategies to ensure a consistent stream of desired, qualified enrollees to preserve an institution's livelihood. Rather than accept a more passive approach toward enrollment ebb and flows, institutions desired a more solidified and structural operational unit that would manage the overall process of attracting, retaining, and graduating students (Huddleston, Jr., 2000).

Anxiety is not a new phenomenon as apprehension over enrollment figures can be traced back to the eighteenth and nineteenth centuries. Until the 1980's, recruitment and marketing was kept separate from those expected to maintain enrollments and process admission applicants. Significant catalysts in history, such as the Servicemen's Readjustment Act (known as the GI Bill of Rights) in 1944, the National Defense Education Act in 1958, and the Higher Education Act passed in 1965 all reflected the federal government's desire for a more educated populace (Hossler, 1984).

The term enrollment management is credited to Jack (a.k.a. John) Maguire and Frank Campanella who were both employed at Boston College (Riley, 2007; Lucido \& Schulz, 2011). This concept began as a response to an increasing demand for a college education due to 1960 s the postwar baby boom. Perceptions began to change as social 
movements of the time advocated that all types of students should have access to higher education.

Early in its existence, some people confused enrollment management with marketing; its actual purpose exists in both conceptual and procedural contexts. Conceptually, the EM asserts a vision for the institution to maintain enrollment stability while demonstrating the set of activities, programming, and approaches the target prospective students. Procedurally, enrollment management represents the programming or activity-based efforts to attract students to maintain enrollment (Baldridge, Green, \& Kemerer, 1982). As the popularity and adaptation of enrollment management spread, campuses began to modify and refine its conception and practice, creating a very fluid model (Dennis, 1998).

Population growth and changing expectation of educational opportunities necessitated a response by institutions. Colleges and universities reacted by developing and diversifying their mass marketing materials, expanding recruitment staff and territories, and utilizing computers more efficiently to track data and manage financial aid packages (Dixon, 1995b).

In the mid-1980s, enrollment management continued to gain popularity and interest but was still too vague to suggest a precise definition. It did, however, continue to include more offices and academic units across campuses for enrollment management planning. During that time, Hossler (p. 6-7, 1984) said that enrollment management planning must oversee the following:

1. Student marketing and recruitment

2. Pricing and financial aid 
3. Academic and career advising

4. Academic assistance programs

5. Institutional research

6. Orientation

7. Retention programs

8. Student services

These eight areas greatly expanded the more simplistic expectation of recruitment, marketing and admission, and they indicated not only the growing responsibility of enrollment management and those who direct its functions but also its linkage with monitoring retention (Dennis, 1998). Even during this time period, the lack of clarity and consistency surrounding the profession showcases a dynamic and complex environment.

\section{Enrollment Management Models}

Four models, listed below, represent the most common types of systems for governing the process of enrollment management; however, no specific model was recommended as the most effective (Dixon, 1995b). Those who are charged with managing enrollments must possess tremendous coordination and management skills while capitalizing on an innate ability to be creative, forward-thinking leaders who analyze problems for positive solutions (Dennis, 1998). Each of the models are listed below to demonstrate the various types of environments in which EMs may function (Baldridge, Green, \& Kemerer, 1982; Clagett, 1991; Dixon, 1995b).

\section{The Enrollment Management Committee}

The enrollment management committee (sometimes also referred to as a marketing committee) functions more in an advisory capacity toward recruitment efforts 
while ensuring cross-collaboration from multiple campus units. Rather than a single designated leader for enrollment management goals, the committee is comprised of multiple individuals representing areas such as academic affairs, student affairs, administration, and other units across campus. An "everyone is responsible for recruitment" stance is expected across campus while the committee focuses efforts on data research and analysis, programming and special event coordination, and communication (Baldridge, Green, \& Kemerer, 1982; Clagett, 1991). For institutions that adopt this model, how are key decisions expected to be made given the shared responsibility?

\section{Enrollment Management Coordinator/Director}

The coordinator model may consist of a Director for Marketing, or more often, a Director for Enrollment Management. The need for an organizational lead to administer enrollment management responsibilities is the primary drive for this model (Baldridge, Green, \& Kemerer, 1982; Clagett, 1991). There are inherent challenges for this type of manager as this change agent must have administrative authority to properly and effectively shape enrollment.

\section{Enrollment Matrix System}

An Enrollment Matrix System suggests that separate units and offices share enrollment management responsibilities based on their role within the enrollment cycle, but a senior-level administrator is still required to oversee the structure and govern priorities. Without re-organizing separate units and offices across a campus, this model encourages an institution to pool together functions and services toward a unified purpose to match enrollment goals (Baldridge, Green, \& Kemerer, 1982; Clagett, 1991). Like the 
Committee approach, this model shares similar challenges as assumptions portend a synergetic environment. Despite pontification toward cooperation, application of these types of models may present difficulties not adequately described in the literature.

\section{Enrollment Management Division}

Finally, the Enrollment Management Division necessitates the most drastic restructuring and re-organization of all the models, but perhaps it reflects a stronger institutional commitment toward making enrollment management a priority to support the overarching institutional mission. Some campuses may feel the need for a drastic reconstitution of offices, units, and service areas as well as potentially re-purposing staff - creating a massive shake-up of the status quo (Baldridge, Green, \& Kemerer, 1982; Clagett, 1991).

The literature openly suggests there is no single, one-size-fits-all approach toward designing and implementing an enrollment management operational structure. Applying an ideal enrollment management strategy is not possible due to the individual attributes and goals of each institution (Dixon, 1995b). In Black's 2004 anthology, Essentials of Enrollment Management: Cases in the Field, a total of eight institutions showcased particular enrollment management strategies to assist the enrollment management leader in his or her efforts. However, none of these institutions utilized the same model in their application of, or approach to, best practices.

\section{Strategic Enrollment Management}

The field of enrollment management evolved further when the phrase "strategic enrollment management" arose in the 1980s as institutions incorporated financial aid into recruitment strategies. Institutions deploying strategic enrollment management practices 
reorganized offices, areas, and units to match desired functional structures. Beginning in the year 2000, a growing interest in partnering with faculty developed. The notion of increasing enrollment no longer dominated the singular purpose of enrollment management practices as strategic enrollment management plans implemented strategies to ensure student success through retention and graduation (Machado-Taylor, Peterson, Taylor, \& Wilkinson, 2007, p. 6).

Even those practicing strategic enrollment management principles do not agree on a singular definition or model for enrollment management (Machado-Taylor, Peterson, Taylor, \& Wilkinson, 2007). This demonstrates the difficulty EMs have in replicating a successful approach or strategy; Black (2004) advises the reader, “...to translate, and where necessary, to modify the strategies and models presented here to fit a unique circumstance" (p. 24).

As institutions attempt to adopt or integrate SEM standards within their enrollment management model, they may face organizational issues and challenges associated with restructuring. How will the individuals within these units adjust to new responsibilities or expectations, and how could that impact enrollment management decision-making? Enrollment management as an organization will be discussed in more detail later in this chapter.

\section{Expectations and Characteristics for Enrollment Management Leaders}

A well-defined and coordinated effort for superior enrollment management is useless without effective leadership. Bold leadership is expected of the enrollment manager, but the institution's president, provost, and/or other upper-level administrators are also significant for the type of strategic enrollment efforts desired. For true 
optimization of enrollment goals, leadership must permeate throughout the organization. Therefore, the environment the EM must administer should reflect a solutions-driven attitude that inspires employees (Black, 2004). This coincides with the collaborative expectations for enrollment managers, but involvement with institutional executive leadership also implies another layer of potential decision-making complexity given the probable differentiation for enrollment priorities.

Expectations of EMs continue to increase and vary, and these demands may depend on the institution. Regardless if enrollment management responsibilities reside with a division leader, coordinator, or committee, Clagget (1991) suggests these managers should attempt to answer specific questions relating to each stage experienced by the student (i.e. through recruitment, enrollment, persisting, graduating) to help guide decision-making. These open-ended questions are listed in Appendix A. The way these types of questions are asked will undoubtedly lead to follow-up tangential sub-questions that could point the EM in multiple directions ultimately inhibiting decision-making.

The typical EM has ten or more years of experience in higher education fields ranging from admissions, the registrar's office, financial aid, student affairs, or institutional research. Additionally, the preferred EM leader holds a doctorate degree (master's minimum) and exhibits a host of personal and professional attributes contributing to his or her ability to effectively and creatively develop and implement strategies for maximization of enrollment (Dennis, 1998; Huddleston, Jr.,2000).

As the field of enrollment management evolves, so do the necessary skills and traits for the EM leader. As college and university presidents seek opportunities for expansion and inclusion with the surrounding community while navigating a complex 
academic environment, EM leaders must follow suit as well (Weaver, 2005). However, the likelihood that experienced leaders with the requisite traits will enter this role is diminishing. Both the Society for Human Resource Management (SHRM) and the American Association of Retired Persons (AARP) indicate the difficulty in replacing retired faculty and staff endures, a difficulty not due to the lack of candidates but finding those with the expected skills (Presswood, 2011).

The desire and expectation for enrollment management professionals to have acquired a terminal degree coupled with over a decade of experience in preferred fields does not match well with the forecast presented by Presswood (2011). How will this incongruence influence the quality and effectiveness of decision-making? It is not unrealistic to anticipate future decision-making challenges for EMs who may not possess these qualifications.

\section{Role of Data/Research in Enrollment Management}

One of the cornerstones of the practice of enrollment management is collecting and interpreting data. Effective enrollment planners are expected to know the internal and external variables that contribute to the successful prediction of enrollment, and the pressure for attracting and enrolling students is greater than ever before (Salomonson, 2005). One of the "core concepts" of Strategic Enrollment Management (SEM) is that "Everything is assessed and measured so decisions are data driven" (Machado-Taylor, Peterson, Taylor, \& Wilkinson, 2007, p. 9).

When asked about what is essential for enrollment management, one of the founders of enrollment management, John Maguire, responded, “...data-driven decision making and fact-based management, linking people and resources to get it done in the 
area of higher education marketing" (Riley, 2007, p. 8). College and university administrators are cautioned to not continue as they have been - making decisions without first analyzing and interpreting the available data to help inform those decisions (Black, 1999).

Thus, the EM leader should not only possess a proclivity toward research but also be able to ask the right questions for data collection. While s/he may not necessarily be required to possess the competency to conduct the research, the EM leader must be oriented toward research-driven decisions (Hossler, 1986). Too few educational leaders devote the necessary time to using data in their decision-making and instead act upon personal experiences, intuition, and anecdotal observations and information. Carpenter and Flowers (2009) suggest that some of these leaders view data analysis with disdain, or at least as an undesirable activity, while others merely view it as too time consuming.

What data should be collected and analyzed? Dennis (1998) suggests a detailed list of reports that should be generated for EMs to review, analyze, and use in strategic decision-making. Those include: Marketing and Enrollment (20 reports); Financial Aid Analysis (10 reports); Retention (15 reports); Surveys and Publications (6 publications); and a Miscellaneous category ( 9 reports) (p. 28-30). The complete list can be found in Appendix B. Although the suggestion was made by Dennis to include a "researcher" as part of the enrollment management team, there was also a recognition that these are only part of the data elements and reports needed for review and that "there will be more requests than there is time in the day to accomplish them" (Dennis, 1998, p. 28). Regardless, the data and information needed for analysis, interpretation and ultimately, 
strategic action taken is as diverse as the potential sources from which they originated (Clagett, 1991).

The responsibilities and expectations listed in the previous section require the EM to not only have access to data and the tools - or resources to gather it - but also to be able to utilize data in the decision-making process. However, many administrators and educators have not participated in any formalized training on using data and may feel overpowered by its complexity. Some EM leaders are faced with inadequate time to gather the data and analyze it (Carpenter \& Flowers, 2009). When asked what recruitment or retention strategies have been successful, "many enrollment managers have no evidence to justify their priorities other than their own intuition, vague anecdotes, general claims made in the literature, and what peers are doing" (Black, 2004, p. 11). Too few retention initiatives and plans are built on data, and many EM leaders have admitted their uncertainty as to which data they should be collecting let alone what decisions should be made as a result (Culver, 2012a).

Who is responsible for gathering or generating the data? Researchers employed in offices of Institutional Research (IR) are certainly one source. Some are expected to help educate the receivers of the information (deans, enrollment heads, senior administrators) concerning the components of the reports received. This requires IR staff to work closely with others across campus to limit confusion as well as prevent data from becoming stagnant and thus ineffective as a tool for decision-making (Johnston \& Kristovich, 2000). Enrollment managers may also solicit help from vendors who specialize in consulting with institutions to maximize their enrollment. 
As one enrollment manager bemoaned, "We don't have anybody on staff that has the time or the expertise to crunch the numbers like they do... They can just do things that we don't have the technical capability to do" (Lucido \& Schulz, 2011, p. 11). The literature seems to reflect a disconnect between the expectation for EMs to make datadriven decisions and the realities of their apparent limitations for accessing, analyzing, and utilizing the data.

Providing accurate, routine, and reliable data to key decision makers is only part of what is needed; whomever within enrollment management is making decisions based on the information provided should also possess an understanding of the contextual properties from where the data was developed. In other words, the data has to be relevant and purposeful but "translated" well by the receiver of the information (Johnston \& Kristovich, 2000, p. 65). However, ambiguity is still a reality as the line between making decisions based on data or anecdotal observations and beliefs is blurred at best (Johnston \& Kristovich, 2000).

Attempting a balance between too little or too much data may hinder the decision-making process as the complexity of doing so can be overwhelming. Even when trying to limit the amount of data for the sake of simplicity, more data may be required to make the best decision. Some issues, such as determining the success rates of scholarship awarding based on class size, ethnicity, retention, academic aptitude of student, and the overall budget may require greater amounts of detail and separation of results to better assess each goal individually. Depending on the recipient of the data, the sheer volume and intricacy of the data may cause analysis paralysis (Anderson, Foley, \& Milner, 2008). 
Utilizing technology can greatly assist in collecting and even interpreting available data, but even the most impressive electronic data analysis tool (which can be extremely costly) is insufficient for all needs. Mining the data requires a rigorous commitment to identify hidden relationships and patterns for the eventual purpose of sharing information and uncovering previously undiscovered knowledge. Unfortunately, most organizations (including the private sector) have data warehouses that contain vast amounts of data but remain "knowledge poor" (Schumann \& Streifer, 2005).

An ancillary incentive for making data a primary source for decision-making is maintaining the perception that senior administrators reach conclusions and/or choose specific strategic paths based on evidentiary justification. Faculty and staff are more likely to respond positively toward action if the existing culture has a demonstrated history of evidence-driven management (Breen, Jenkins, \& Lindsay, 2002).

If data is truly to be considered “...the lifeblood to successful student recruitment and retention," (Culver, 2012b), then the literature has reflected an ambiguous reality for enrollment managers to embrace. The complexity surrounding data collection, data analysis, and the decision-making that should occur as result of the data yields an uncertain direction for leaders to follow.

\section{Professional Associations and Consulting Firms}

Considering that no single enrollment management model exists that perfectly aligns with every institution's mission, organizational structure, or enrollment goals, campus leaders must be able to apply the key concepts and principles in a format appropriate to their institution (Penn, 1999). A variety of professional organizations comprised of non-profit associations to commercial businesses have offered services to 
assist enrollment managers in planning and strategic efforts. Most of these private vendors offer consulting for recruitment publications, marketing strategies, computer information systems, and operational or managerial recommendations (Huddleston, Jr., 2000). Due to the complexity of the enrollment management environment, an increasing amount of institutions are choosing to solicit consultants and organizations to address these needs (Lucido \& Schulz, 2011). For a review of these associations and vendors indicating the type of services and potential consulting available for enrollment managers, refer to Appendix C.

These organizations (AACRAO, ACT, College Board, Hobsons, Noel-Levitz and Stamats) merely represent a sample of the potential associations, agencies, and companies offering assistance for institutional leaders desiring improved enrollment management practices to help direct their decision-making. In addition to the six mentioned above, some companies offer numerous technological systems (e.g. CRM, Customer Relationship Management systems) for a purchase price in the hundreds of thousands of dollars to say nothing of yearly maintenance costs. Some example products include document capture and imaging, automated workflow of documents and processes, and intricately detailed personalization of communication.

These systems bring the promise of streamlined operations, improved efficiency, and higher student satisfaction. Similar to the assumption that no perfect enrollment model exists for all institutions, there is no guaranteed technological system that applies to all institutions. The onus still falls upon the enrollment managers who will need to devote the necessary time, financial resources, and commitment to determine if they have 
a need that may be addressed by a technological solution in their decision-making practices.

\section{Future of Enrollment Management}

Whether or not a campus possesses a formal enrollment management structure, the advent of this philosophical approach has made institutional leaders habitually more assiduous in their intentionality toward shaping their student body (Dixon, 1997a).

Planning and preparing for enrollments at an institution has been compared to predicting the future. While forecasting enrollment growth or decline may be part of the enrollment management equation through an understanding of how state or national policies as well as how demographic, economic, and technological trends impact enrollment, decisions may not be possible without research. One of the fundamental truths surrounding research is the understanding that the higher education environment is changing (Layzell, 1997). Enrollment managers are compelled to monitor and adhere to the dynamic nature of higher education by developing and expanding their programming as well as meet the technological expectations of current and future students.

Dennis accurately predicted in 1998 that institutions would see their enrollment management programs develop and alter from the more traditional undergraduate and graduate programs to include "corporate outreach programs, distance learning programs, combined degree programs, and collaborations with local colleges and universities, and international contracts and collaborations" (p. 19). Aside from these forecasts, Dennis again promised that no matter what plans may be in place at the time, enrollment managers should always expect and prepare for change. 
Technology impacts many facets of higher education, and the areas and functions of enrollment management face both challenges and opportunities due to technology's influence. Today's students demand real-time and even any-time services, and they expect customized responses. Enrollment managers are confronted with the fact that there will be fewer face-to-face interactions between students and the staff. Depending on the institutional mission and values, EMs will have to react accordingly to these demands and expectations keeping in mind aspirations for quality student engagement and interaction. Failure to respond with appropriate strategies could have significant consequences for the institution in terms of low enrollment, retention, or graduation numbers (Black, 1999).

As has been demonstrated earlier in this chapter, enrollment management professionals have not been pursuing strategic enrollment initiatives alone. Reliance on the services and consultation of commercial vendors has aided in the process. In fact, enrollment management itself has become a blossoming business due to the importance of enrollment equating to tuition revenue (Hossler, 2004). However, as many institutions rely on the next cycle of enrollment to determine if they are able to "make or break it" financially, the question has to be asked whether this dependence on third-parties for decision-making or data analysis is sound educational practice (Lucido \& Schulz, 2011). The complexity of the ever-changing environment may be altering the traditional engagement of EMs in planning and execution efforts. Perhaps it is not enough to simply be enrollment managers; the future may demand these managers to become transformational leaders at their institution (Black, 1999).

\section{Organizational Theory in an Enrollment Management Context}


Although presenting the historical background and developing elements of enrollment management help demonstrate a surface-level perspective, understanding the environment of enrollment management from an organizational theory context will reveal deeper realities experienced by the EM. In this section, specific components of organizational theory that influence this environment will be listed, including culture, governance and leadership, planning and change, open systems, and power and politics.

\section{Culture}

Tierney (2008) utilizes a metaphor of how Sherlock Holmes solves and investigates mysteries to describe the complicated and complex environment of an institution of higher learning. Holmes seeks to analyze ostensibly unrelated events and individuals hoping to discover links and associations to provide clarity to a situation. What Tierney suggests through the Holmes metaphor is that an institutional culture consists of much more the facts and figures of a bottom line. Tierney (2008) claims,

"An organization's culture is reflected in what is done, how it is done, and who is involved in doing it. It concerns decisions, actions, and communication on both an instrumental and a symbolic level” (p. 24).

The enrollment management environment possesses its own culture with all of the attributes inherent of that culture including adherence to Tierney's statement that "Culture is in constant flux and reinterpretation" (p. 2). These influences may further complicate the EM's ability to maximize his or her decision-making opportunities.

Strategic enrollment management practices and philosophies follow and embrace aspects of culture as it applies to organizational theory. An organization's culture can often encompass shared values, concepts, beliefs, traditions, rituals, and other facets of 
the particular institution that apply meaning to individuals within the organization itself. Culture has tremendous influence over the actions and decisions made by individuals within an organization as its values and meaning may permeate throughout (Morgan, 2006). The culture of an institution, especially its espoused vision and values, can tremendously impact the way decisions are made as well as the decisions themselves.

Despite the direction and boundaries culture may impose upon an organization, it does evolve. Its values, beliefs, and even traditions will alter over time, especially as approaches toward external influences may expand (Kuh, Schuh, \& Whitt, 1991). Adding to the complexity of institutional culture is the presence of multiple subcultures which may offer competing values and assumptions, and there is no question subcultures within enrollment management will also affect decision-making as they may conflict or compete with one another, and, at times act in disharmony (Kuh, Schuh, \& Whitt, 1991; Morgan, 2006).

Likewise, individuals within these subcultures may not understand the perspectives of the others which can create confusion to say nothing of a value system divergent of the proclaimed institutional values. This demonstrates yet another layer of organizational intricacy and complexity as EMs attempt to navigate within their own cultural environment while balancing the values, assumptions, rituals, etc. of others. This reality, coupled with the already fluid, dynamic, and aggressively changing surroundings within enrollment management indicates a strong possibility of uncertainty toward decision-making as each EM attempts to incorporate and/or balance these potential influencers.

To fully embrace the impact of culture on organizations, traditional views of 
management must be suspended. More traditional and conventional models of institutional management feature the more common "management by objectives, goalbased planning, organizational charts, communication channels, and hierarchical structures..." However, this type of organizational rationality is unrealistic primarily due to the real-life uncertainty and ambiguity found in organizations. Kuh and Whitt (1988) felt this duality between traditional structures and ambiguous realities were "inherent in organizational life and that differences between what "should be" and what is are not evidence of problems that must be fixed" (p. 4). These authors recommend institutional leaders discontinue their devotion toward organizational rationality and embrace the notion of unpredictability.

Although it may be difficult to determine or describe a common cultural model for enrollment management as well as the subsequent types of decisions made by respective EMs, Kuh, Schuh, and Whitt (1991) suggest that members of an organization hold the sentiment of value as being the most important component in the decisionmaking process. Additional influential factors include the organization's history, sometimes portrayed as its "story." A member's connection and commitment to the organization's story can direct action as well as non-action due to their beliefs and devotion to the organization's ideals (Clark, 1972, p. 178).

\section{Governance and Leadership}

Although clear lines of subordination are documented at each institution of higher learning, the varying concept of governance permeates through multiple hierarchies. All institutions receiving federal funds are required to follow federal laws and legislation, and Oklahoma public institutions receiving state appropriations are required, by the 
Oklahoma Constitution, to follow Oklahoma State Regents for Higher Education

(OSRHE) guidelines and policies (Oklahoma State Regents for Higher Education, 2007).

AACRAO provides guidance and oversight in multiple areas within enrollment management, and college and university registrars rely heavily on this organization to provide best practices and standards especially in the areas of transcription of the academic record (Hossler, 1984). The role of the registrar has endured transitions over the years as it was once a position primarily devoted to the care and stewardship of student records and academic policies. Now, it functions as a key contributor in strategic planning and decision-making practices of enrollment management (Presswood, 2011).

As mentioned in previous sections, oversight of enrollment management functions, and therefore their governing structures, vary by institution. While some colleges and universities employ a vice president or dean with the responsibility of managing an entire division, others have cross-campus committees expected to develop collaborative enrollment management functions and responsibilities. One of the main purposes of sharing responsibilities for oversight of enrollment management functions is to create a sense of ownership while developing the members' level of commitment (Machado-Taylor, Peterson, Taylor, \& Wilkinson, 2007). Tierney (2008) suggests institutions should embrace the dynamic and fluid nature that a responsive and innovative governing structure brings; however, the effort for governing entities to function in full accord remains a daunting task as "thousands of hours of consultation and conversation" need to take place prior to a final and satisfactory decision (p. 120).

March (1991) suggests that most people conceptually view organizations as hierarchies where the higher positions supervise the lower ones, and policy and practice 
control implementation. He disagrees with this perception, stating, "Such portrayals seem, however, to underestimate the confusion and complexity surrounding actual decision making" (p. 107).

Regardless of the format or title of the enrollment management primary leader, the notion of shared governance is still proclaimed to be the preferred method of management, especially as it applies to decision-making in higher education. Those expected to engage in the process include - faculty, administrators, and various staff. Given the divergent responsibilities these areas represent, the potential for disharmonious decision-making is high, adding to the complexity of the EM's environment (Keller, 2001; Simplicio, 2006). Keller (2001) suggests leaders should consider making symbolic decisions, rather than well-researched ones, if the demands of the position become too complex. Simplicio (2006) relates the lack of consistency of a dominant governance strategy, "As a result, due to the complexities and intricacies of these many factors, no definitive prototype can be offered as a model for campuses to follow" (p. 764).

Just as cultural influences can impact decision-making, regulations, guidelines, and laws communicated through or by governing organizations, to say nothing of the internal institutional structure, will also encircle the EM in a cloud of complicated parameters that may affect his or her judgment.

\section{Planning and Change}

A tremendous expectation is placed on enrollment managers who may also be labeled as enrollment planners due to their involvement with strategic planning. Campuses simply do not stand still, even if institutional leaders want them to maintain status quo. Change does not come easily, however, and the planning involved in 
incorporating a new vision or strategy may frustrate leaders as implementation often takes considerable time, especially on a college campus (Tierney, 2008). Enrollment management strategic planning models exist, but similar to other facets of enrollment management, "The planning model and process has to be tailored to the institution in order to be successful” (Machado-Taylor, Peterson, Taylor, \& Wilkinson, 2007, p. 12).

Whether it is described as strategic planning, reengineering, or redesigning, those who initiate it must be motivated and willing to contribute. Strategic enrollment management (SEM) planners should be leaders who are "guided by solid research and organizational needs" (Dolence, Lujan, and Rowley, 1997, p. 219). Unfortunately, few campuses conduct research on expectations of incoming students, which severely hinders their ability to potentially react and address current and future needs. Likewise, many institutions neglect to evaluate the results of any change and implementation. Without data, these leaders are unable to accurately determine whether the change was worth the investment (Black, 2004). The literature seems to indicate that enrollment management professionals may be either unable or unwilling to incorporate or utilize data effectively in their decision-making processes, especially as it applies to informing strategic planning and change efforts.

\section{As an Open System}

Institutions of higher learning survive through reliance and dependence on the external environment, and the enrollment management atmosphere embodies this notion. Burke (2007) states, “An organization is open because of its dependence on and continual interaction with the environment in which it resides" (p. 49). In an almost symbiotic relationship, EMs must strategize with faculty, staff, and administrators from units across 
campus, but they must also include external members of the surrounding community that may be located in targeted areas for recruitment (Morgan, 2006). The external communities represent yet another element that must be considered during the EM's efforts to make decisions.

SEM planning requires cross-collaborative efforts, so the format mirrors this concept and clearly portrays the complex environment in which leaders must maneuver. Prior to calling it an open system, early literature concerning enrollment management documents the need for EMs to constantly be analyzing demographics of the institution's marketing base as well as a call for leaders to monitor policy trends both at the federal and state levels (Baldridge, Green, \& Kemerer, 1982; Dennis, 1998; Gumport, 2001; Hossler, 1984; Hossler, 1986).

\section{Power and Politics}

A considerable amount of life within an organization is spent by members negotiating their diverse interests within political contexts while also engaging in power dynamics to assert themselves in the overall structure. Political interplay ensues as a result of individuals attempting to establish their authority or control over each other, parts of the organization, or the organization itself. Regardless, organizational politics will arise whether people are intentional about it or not (Morgan, 2006). As enrollment leaders attempt to create or implement an SEM plan, for example, they will have to balance it against dynamic and open-ended platforms that may be built upon fear of change and the defense of respective interests, goals, or traditions. In short, EMs should be prepared for each decision to be challenged (Dolence, Lujan, and Rowley, 1997). 
In any organization, leaders will inevitably enter into a political arena occupied with individuals determined to advance their agendas. Enrollment managers will not only have to be purposefully collaborative in their own areas, across campus, and with external constituencies, but they will also find their resolve tested as entrenched individuals may challenge decisions in order to protect their own interests. How will they respond to such challenges?

\section{Decision-Making}

\section{Organizations and Decision-Making}

The functions of an organization rely on the ability to process information. Regardless of organizational design, the decisions that are made as a result of the information being gathered, deciphered, inspected, or analyzed help shape and guide daily practice as well as provide direction. Morgan states, "Organizations are information systems. They are communication systems. And they are decision-making systems" (Morgan, 2006, p. 76).

One of the main expectations for organizational leaders is to make effective decisions. Administrators within institutions of higher education would be wise to recognize the influence of culture upon those decisions, especially because of increasingly obscure contextual environments due to decreasing funds and resources. Through both formal and informal conversations, ranging from impromptu hallway rendezvous to structured unit meetings, decisions will be made (Tierney, 2008). How decisions are made within the organization will vary by the individual, team, or potential hierarchy of the institution, and members must learn the "rules" of the organization (Carley, 1996, p. 235). 
De Feis and Rahman (2009) suggest that despite the abundant varieties and options associated with decision-making models, practitioners remain stagnant in their ability to make strategic decisions. The authors felt this incapacitation was due to the environment's complexity, referring to it as the "number and interdependencies of different components that exists in any decision-making process..." (p. 45), as well as time limitations associated with the decision.

More often than not, decision-making in organizations appears to consist of routines and rule-following. People generally follow the directions they are given, adhering to any prescribed policies, procedures, and regulations associated with their job. Any decision made is most likely derived by some standard, cultural norm, or institutional mandate. However, the individuals making these decisions will also evaluate alternatives and make choices based on the aforementioned expectations. These decisions will vary depending on the individual's identity as well as the situation (March, 1991). Whenever decision-makers face "nonroutine matters" for which there is no prescribed method to address new issues, managers will tend to make decisions based on perceived expectation. In other words, they will make choices or take action for what they think "they are supposed to" in order to meet their boss' expectations (Jackall, 2010, p. 81). These arguments flow directly into the larger discussion presented concerning the complexity surrounding enrollment managers' ability to make effective decisions.

In the following section, the strategies and approaches toward decision-making will be examined more closely. These strategies include rationality, Herbert Simon and bounded rationality, satisficing, and the garbage can model. Both satisficing and the 
garbage can model will be discussed specifically because of their applicability in enrollment management and overall higher education contexts.

\section{Rationality}

The concept of rationality suggests that action occurs after consideration of all possible choices; the resulting choice is made with the expectation of optimal results. Simply speaking, decision-makers employing the rational model know their objectives, ascertain and evaluate the alternatives to achieve the objectives, and select the best one (De Feis \& Rahman, 2009; Heath \& March, 1994; March, 1997). However, March (1991) reveals limitations of the concept of rationality as an appropriate model for decision-making in organizations. Decisions are bounded by individual limitations and organizational constraints that prevent an optimal resolution.

Although the rational model as well as variations and expansions of its application in organizational decision making have been recognized for the better part of sixty years, March (1997) and Choo (2006) recognize its inadequacies in terms of adhering to the tenets of rationality - the requirement of identifying all potential alternatives, recognizing each one's consequences - and a known desired outcome is too demanding to realistically expect. As rational choice has been further studied over the years, a stronger recognition of its limits and inconsistencies has yielded awareness of a bounded form of rationality. Zey (1992) edited and published a collection of models and concepts that expressly critique rational models highlighting the subjectivity that individuals place on the value of selecting an optimal choice.

\section{Herbert Simon and Bounded Rationality}


A 1978 Nobel Memorial Prize winner in Economic Sciences, Herbert Simon viewed economics as an area worthy of study and research especially from an openended empirical framework. He felt that most of the economic practitioners who dominated research in this area had predispositions toward true rationality (Anderson, 2001). Bendor (2003) offered words from Simon to explain his views on how humans are limited in their ability to maximize options:

"...the capacity of the human mind for formulating and solving complex problems is very small compared with the size of the problems whose solution is required for objectively rational behavior in the real world - or even for a reasonable approximation to such objective rationality" (p. 435).

Economic theorists and psychologists who study behavior have advocated the existence and strength of rationality for quite some time. However, that does not mean its features are above criticism. Simon became a staunch critic of the pure rational choice models as he felt humans do not always operate from this logical and linear approach toward making decisions but instead utilize a bounded rationality method. He felt that realistically, humans do not possess full access to the complexity of the environmental factors nor an effective cognitive system in order to maximize every real-life decision (Campitelli \& Gobet, 2010). The theory of bounded rationality therefore suggests that individuals can and will make decisions based on practical and available calculated factors, and this information will be incomplete in scope. Nevertheless, despite not having a fully stocked cache of data or information available at the time a decision is desired or required, reasonably solid decisions can be made. 
Simon suggests that bounded rationality exists as a limitation of the decision maker's capacity to understand, comprehend, and choose to the sheer density and depth of the potential choice needed to be made (Bendor, 2003). Although this may be interpreted as a judgment on the fallacies of human intellectual prowess, the author contends it is merely Simon's perception of the role that environment and context plays upon the decision to be made. This description may very well apply directly to enrollment management environments as well as the ability of its leaders to process all of the data intended to drive their decisions.

A list of attributes may be gleaned from Simon that illuminate potential qualities impacting critical decision-making. The first is the ability to receive discriminatinginformation. Each individual possesses filters that help to process and digest information. Additionally, a higher-level ability to frame that information is needed. Being conscious of the influence of many internal and external factors upon a decision within an organization is essential to making rational choices. A third attribute is a fairly slow and limited ability to process information due to physiological limits. In short, it takes time to weigh all known or recognized angles related to the person's propensity to make decisions. The fourth is a recognition that humans are slow to make calculations. Understanding the benefits and consequences of decisions is also a time consumer. Fifth, overall human memory fades fairly quickly and must therefore reconstruct images and information while lacking real accuracy. The sixth and final attribute is the limitation of short-term memory. Taken together, all of these factors greatly impact the individual's ability to make decisions (Bendor, 2003). These attributes appear to match up exceedingly well with the limitations found in an enrollment management environment. 


\section{Satisficing}

The notion that human beings satisfice their decisions is derived from the assertion that psychological assumptions associated with rational choice theories are unrealistic. Humans regularly defy the tenets and principles of rational choice. In more contemporary behavioral economic research, rational choice has been viewed as more and more implausible (Lehman, Lyubomirsky, Monterosso, Schwartz, White, \& Ward, 2002).

Simon first suggested the concept of satisficing nearly a half-century ago and introduced it as a means of more accurately and realistically explaining how humans make decisions. He claimed that the goal of maximizing choices to find an exacting and optimal solution was overwhelmingly unrealistic. Additionally, he asserted that humans will look for a solution that is "good enough," and there is a great deal of variance in the levels of how that choice meets the expectations. However, the point is that it is meteven though it is often not the absolute best choice. Satisficers are not driven by the goal to discover the maximized and optimal solution but merely an adequate one (Lehman, Lyubomirsky, Monterosso, Schwartz, White, \& Ward, 2002, p. 1178).

Additional research indicates that people may actually prefer to have fewer options to choose from when making a decision. A possible reason may be that individuals do not want the regret of not choosing an option that may in later reflection seem more desirable. Another reason is that individuals do not have the capacity to properly store and examine an exhaustive list of options. For example, selecting a choice out of five or six options is manageable, but having to choose from a selection of 40 options can be truly intimidating. Therefore, people may be even less focused on a 
desirable choice and instead opt for a less than august alternative rather than face a possible arduous choosing process (Lehman, Lyubomirsky, Monterosso, Schwartz, White, \& Ward, 2002).

Considering the recommendations and/or expectations for EMs to utilize a multitude of sources to help them make decisions, as well as the personnel influencers potentially integrating their own assessments and desires, the concept of satisficing appears to be a legitimate perspective for enrollment management decision-making.

Simon also used the notion of satisficing and applied it within an organizational context. He suggested there are six kinds of rationality that can be found within the organization: “1) Objectively rational; 2) Subjectively rational; 3) Consciously rational; 4) deliberately rational; 5) Organizationally rational; and 6) Personally rational." The "satisficing man" within the organization is the individual who operates within a rationally-minded (perhaps not in action, however) organization but who functions from a practical perspective (Brown, 2004, p. 1242).

Simon breaks down a comparison between what he calls the "economic man" and the "administrative man." It is the economic man who enters into a decision-making process with the intention of maximizing — selecting the optimal choice from all possible angles while viewing the complexity of the situation. The administrative man, on the other hand, satisfices his choices by selecting a "good enough" option while viewing a much more simplified version of the situation. Simon suggests it is this quality of the administrative man which helps him make a choice instead of possibly being paralyzed from evaluating a nearly endless branch of options like the economic man (Brown, 2004). 
Henden's (2007) article concerns the possible motivation of satisficing. Some have claimed that these individuals have a propensity to be moderate or reserved in their approach toward problem solving. However, the author also suggests that there may not be a reason why these satisficers settle on their choice but that they may have a warped or simply enervated ability to recognize excellent choices from mediocre ones. Therefore, the individual may satisfice as a result of being a moderate or feeble interpreter of a scale of worthwhile choices. How would the enrollment manager react in similar situations? Would s/he settle on a choice based on a purposeful approach toward solving problems or potentially due to flawed evaluation of options?

Critics may label those who satisfice as not striving to meet high standards, are too content with the status quo, or may even obstruct quality research. However, advocates of satisficing believe that approaching a problematic situation with a satisficing notion may actually help shape educational reforms from a realistic and achievable dimension. Kerdeman (2009) asks: How can an organizational entity, such as those within the various models of enrollment management, claim to adhere to certain standards and levels of expectation if its members choose good-enough options instead of seeking and possibly acquiring the ideal option?

Previous studies have indicated that satisficers may have a quicker response time of taking action upon a given situation. In some heuristic environments, that may be a sought after trait. At the same time, however, satisficers also have the potential to be susceptible to harsh criticisms of cognitive limitations (Ahluwalia, Keil, Tiwana, \& Wang, 2007).

\section{Garbage Can Model}


The garbage can model was introduced as a model to help elaborate upon organizational decision making, specifically those structured as organized anarchies. Institutions identified as organized anarchies are labeled as such due to "severe ambiguity" of their goals or objectives, means to accomplish them, and who should be included or drive the decision-making process. This theory focuses "less on the details of individual decision making and more on the aggregate flows of people, problems, and solutions through organizational networks" (Padgett, 1980, p. 583).

Contrary to a linear approach of decision-making, the garbage can model of decision-making implies that an organization's choice opportunity is a complex mixture of problems and solutions created by the organization's members that are representatively dumped into a garbage can. Multiple cans may exist, and the amount and mixture of the type of garbage varies on what type of refuse is being produced at that particular time. A decision is reached only through a complex interplay of four factors: problems, solutions, participants, and choice opportunities (Cohen, March, \& Olsen, 1972; Fioretti \& Lomi, 2010; Lane, 2001).

A given problem and subsequent solution are not exclusively linked in this model but are respectively independent of one another. Not only can solutions address multiple problems, but some recent researchers of this model suggest that postponing a decision or even delegating or deferring decisions can be of benefit to the organization (Fioretti \& Lomi, 2010). Due to the fluid nature of this model, timing and flow of information may raise issues with decision-makers. This model "stresses the importance of limiting data to that which is useful and relevant to a decision, and only providing it to those actively involved in the decision-making process" (Lovata, 1987, p. 148). The complexity and 
blend of problems and solutions may fit into the organizational construct of enrollment management as EMs may be faced with decision-making scenarios that complement garbage can methods.

\section{Pairing Satisficing and the Garbage Can Model to Enrollment Management}

The preference for enrollment management leaders to proclaim the importance of utilizing data in their decision-making, as well as the limitations associated with its actual use, matches up well with the notions of satisficing. Despite the demand for enrollment managers to possess the necessary foundational knowledge base to not only be able to filter through massive amounts of data but also access and generate the data to make the best decision available, challenges including being overwhelmed by data, technology constraints, and time and resource limitations remain (Cooley \& Shen, 2008; Kenny, 2009).

Considering how enrollment management is expected to change and/or adapt to new strategies, its culture will undoubtedly be affected. Simon (1991) presented his thoughts on the role of bounded rationality as organizations consider change. He felt that humans already have limits in terms of the ability to adapt to complex organizations and environments, so as the organization assumes new members, it is important to understand how the limits of human rationality impact an organization's desires for change. As an organization's members, or its representative body, take on new members, a fundamental change in the representation has already taken place.

In terms of decision-making desired by the organization, Simon (1991) suggests that if organizational leaders are steadfast in their desires to enact change, it would be quicker and more cost effective to import individuals who may have like-minded beliefs 
or approaches toward the desired change. Rather than trying to instill existing members who may potentially serve as a contrarian, the rationality behind decisions may be less bounded if new members are brought in to embrace the new organizational goals. Considering how each educational institution functions as an organization comprised of a multitude of sub-organizations and cultures, understanding how rationality and bounded rationality may or may not be visible as a decision-making model will help enrollment managers as well as educational practitioners expand their understanding of how institutional decisions are made.

March and Simon (1958) suggest that organizations should recognize the cognitive limits of its members' individual "wants, motives, and drives," and their limitations in "knowledge and in their capacities to learn and to solve problems" (p. 136). The evolving and changing landscape of enrollment management coupled with the increasing expectations and demand for enrollment targeting indicate the theory of satisficing may very well be prevalent for enrollment managers.

Organizationally, higher education has been viewed to function as a loosely coupled collective. Although this type of model may garner positive or negative opinions based on subjective scrutiny, this type of flexibility within the organization allows it to better adjust and adapt. Loosely coupled models such as the garbage can model fit quite naturally with educational systems, especially universities (Lutz, 1982).

Riley (2007) advocates Cohen, March, and Olson's (1972) suggestion that the garbage can model matches up well within higher education organizational settings, especially enrollment management, due to the varying and loosely aligned units of the 
university. This imprecision allows for a permeable and anarchic flow of decisionmaking processes. Riley further explains:

"In a garbage can model process, there are exogenous, time-dependent arrivals of decision opportunities, problems, solutions, and decision makers. The logic of ordering is temporal rather than hierarchal or consequential. Problems and solutions are attached to choices, in large part because of simultaneity" (p. 25).

\section{Conclusion}

The growing field of enrollment management remains a fluid and dynamic structure, and its leadership structures vary from committee chairs to divisional vicepresidents. Tremendous demands have been placed on these leaders as enrollment goals are expected to be reached through campus-wide collaboration efforts while utilizing extensive amounts of data to drive the decision-making process. While internal staff members or departments may aid in data collection, many institutions solicit the assistance of outside vendors to identify and gather information to guide enrollment management leaders. A complex environment consisting of politically-driven administrators, faculty, and staff may complicate coordination and strategizing efforts. The literature indicates that EMs, faced with multiple challenges to their decision-making process may make decisions embedded in bounded rationality (based on Herbert Simon's theory of satisficing), or choose a "good enough" solution rather than an optimal one. This study further considers the available literature on bounded rationality, with specific focus upon enrollment management leaders in their complex environment. 


\section{CHAPTER III}

\section{METHODOLOGY}

In this chapter, the methodology associated with the purpose of this study is presented in a design proper for this type of investigative inquiry. Crotty (2003) describes this process as developing a "plan of action" (p. 7) where the guiding epistemology informs the theoretical perspective and then flows into developing the methodology and methods. Additionally, the problem statement, purpose of the study, and research questions will be revisited. A description of the procedures and methods is presented including details associated with the study sites, participants, as well as the data to be collected and analyzed.

\section{Statement of the Problem}

As a growing entity within higher education organizational structures, enrollment managers (EMs) are primarily tasked with projecting, recruiting, and retaining the student population of their campuses. These enrollment managers are expected by institutional presidents as well as through industry standards to make data-driven planning decisions to reach their goals (Baldridge, Green, \& Kemerer, 1982).

However, despite the availability of data, some enrollment managers revealed a different reality from traditional, rational decision-making models. Their experiences reflected incomplete, fragmented, and ambiguous solutions that impacted the overall 
quality and efficiency of decision-making within the enrollment management areas (Riley, 2007).

Considering that EMs are expected to incorporate a campus-wide and collaborative effort in their plans, as well as the vast amounts of data in the decisionmaking process, this incongruence may exist due to complex factors within the enrollment management environment that may inhibit rational decision-making.

\section{Purpose of the Study}

The purpose of this study was to explore the factors that may contribute to or influence the decision-making process of enrollment managers working in two and fouryear public colleges and universities located in the Midwestern United States; the study also looked specifically at how EMs use available data. Considering not every higher education institution contains bona fide enrollment management units, divisions, or teams, only those institutions who utilize those formal functions were explored in this inquiry.

\section{Research Questions}

1. How are strategic enrollment management decisions made on a campus that utilizes an enrollment management system?

2. As enrollment management professionals make decisions, what do they consider?

3. How do expectations relate to decision-making?

\section{Overview of the Study Design}

When researchers embark upon qualitative inquiries, they seek subjective and emerging responses to respective topics. Contrary to the concise and generalizable data that quantitative studies present for analysis, qualitative studies allow the researcher to 
present findings that tend to be more expansive, in depth, and detailed in terms of unfolding the data to the reader. Particularly prudent to realities contained within higher education contexts, qualitative studies can help practitioners "make sense" of their world through exploratory findings (Major \& Savin-Baden, 2010). Due to the efforts to illuminate additional understanding about the environment of leaders who operate in an enrollment management context, this study employed the basic research strategy as described by Patton (2002).

\section{Guiding Epistemology for This Study}

Creswell uses the term "worldview" (p. 6) to articulate how one generally views the world in a research-based context. Crotty (2003) utilizes the term "epistemology" and explains it as simply demonstrating how one looks and understands the surrounding world, or, "how we know what we know" (p. 8).

The guiding epistemology for this particular study is constructionism (sometimes also referred to as social constructionism). Contrary to an objectivist viewpoint, which asserts that meaning exists without engagement with any external source, those who possess a constructionist epistemology posit that knowledge and meaning is constructed by the interaction and engagement of humans upon objects in the world. Crotty (2003) astutely defines it as:

“...the view that all knowledge, and therefore all meaningful reality as such, is contingent upon human practices, being constructed in and out of interaction between human beings and their world, and developed and transmitted within an essentially social context" (p. 42) 
Reality, meaning, and knowledge are therefore constructed by the individual and applied to an object or situation in one's life (Patton, 2002).

Crotty (2003) expands upon the constructionist view through his description of social constructionism, or more specifically, the impact of one's culture upon making meaning of experiences. Culture is seen as "the source rather than the result of human thought” (p. 53). Humans apply meaning through a social context, and all experiences are deemed as meaningful realities in social constructionism. Behavior and evaluation of experiences can be traced back to cultural influences, and these influences provide the lens for assessing, valuing, and applying meaning to each context. Under this premise, I investigated the individual experiences of enrollment managers in an attempt to capture each one's perspective on his or her reality.

In its truest form, constructionism recognizes that there is no single reality. Multiple realities not only exist, but can also contribute to a tremendous amount of diverse interpretations and understandings (Crotty, 2003). Considering the varied realities experienced by assorted enrollment managers at their respective institutions, this epistemology reflects the range of interpretations of their decision-making realities. The constructionist approach recognizes and values these unique perspectives as well as how the making of meaning applies to each circumstance or condition. The individual connects and interacts with the "object," (p. 48) and it is the interpretation of applied meaning which follows the connection.

This research study was one of exploration, discovery, and immersion into the experiences of those working in areas within enrollment management. The type of method best suited for this form of research was a qualitative inquiry. As it was my 
intention to be able to share the experiences and perceptions of the study's participants, a qualitative inquiry allowed for thick, rich details which helps reveal their realities (Patton, 2002).

\section{Influences on the Study and Theoretical Framework}

Although my own experiences concerning decision-making in an enrollment management context greatly affected the overall construction of this inquiry, this study was also influenced by perspectives and methods found in Riley's (2007) dissertation of a similar topic. While his quantitative study measured the decision-making effectiveness of a particular institution's enrollment managers, this qualitative study sought to reveal the decision-making experiences of enrollment managers of multiple institutions and contexts.

This study also utilized the theoretical framework used in Riley’s (2007) study, Herbert Simon's model of bounded rationality and satisficing as the lens for considering data from this study. While Simon's bounded rationality theory helped explain how decisions are made by the individual, March, Cohen, and Olsen's garbage can model also served as an additional perspective for the types of complex and inter-related decisions made by enrollment management entities (Cohen, March, \& Olsen, 1972; Lane, 1980). The applicability and usefulness of each theory was determined and discussed a priori after data had been collected and the analysis completed.

\section{Procedures and Methods}

Information obtained for this study was gathered from individual interviews, documents and artifacts, and observations. Enrollment management leaders from a total of six institutions of higher learning, located within the Midwest region of the United 
States of, were targeted as sources for data gathering. Exploration, immersion, and evaluation of all sources of data were conducted using a variety of qualitative methods as described in a later section with the aforementioned theoretical perspectives as lenses. The purpose for the varied methods of collecting, observing, presenting, and analyzing data is called developing triangulation, which Patton (2002) suggests helps strengthen a study through the diversification and integration of data sources, theories, and methods.

\section{Study Sites}

A total of six higher education institutions located in the Midwest Region of the United States were chosen as study sites. Each of these sites was expected to produce thorough and distinctive perspectives through their participants' responses to the research questions. The respective institutions were selected based on key, selective characteristics. First, each one is currently an active member of the Southern Association of Collegiate Registrars and Admissions Officers (SACRAO) which is an affiliate of AACRAO. SACRAO institutions are committed to cooperation among member institutions especially in the areas of admissions, registration, and records - areas or departments that traditionally fall under enrollment management purviews. Secondly, these colleges and universities were chosen for their diverse missions and desired student populations. Finally, these schools were preferred due to their varied enrollment management-based organizational structures and geographical proximity.

Southern Regional College (pseudonym), or SRC, was selected as a potential site location due to its multi-campus presence which presented an intriguing environment from an enrollment management decision-making standpoint. The two-year, public institution typically attracts and serves students who originate from rural areas, but 
college administrators and enrollment management planners must be cognizant of increasing associate degree attainment while guiding their students toward transferring to potential four-year institutions residing in various locations in the state. Representatives from their primary campus consistently maintain an active role in the field of enrollment management, including executive positions, in SACRAO. Given their history of cooperation and commitment toward advancing opportunities for educating students, SRC enrollment management leaders were expected to provide rich data to be analyzed.

Based upon their location and student population, Sloan University (pseudonym), or SU, also presented a distinctive setting from other institutions in their state. Located near a military base in an expanding but initially rural city, SU's enrollment management units appeared to focus primarily on attracting and admitting students. Considering their apparent separation from other units typically found within enrollment management entities such as the registrar and financial aid offices, how their EMs make decisions as well as their sources utilized to help inform the decisions enhanced the quality of data gathered.

The largest institution of the selected study sites, The University of the Prairie (pseudonym), or UP, is an institution that in recent years has undergone dramatic changes in their enrollment management areas both in personnel and function. Their cultural and organizational changes yielded insightful findings within the enrollment management context. Possessing greater resources, as well as perhaps more intricate enrollment goals given their state and regional recruitment efforts, UP was expected to provide a comprehensive depiction of enrollment management decision-making processes. 
Garden State College (pseudonym), or GSC, represents the last two-year institution on the study site list. Positioned in a metropolitan area, GSC serves a diverse student body population as well as maintaining strong connections with a nearby military base. From an enrollment management perspective, the college appears to possess fewer units than some of the literature suggests should be included in enrollment management. Therefore, their location, coupled with the expectations for students and community leaders, and their smaller organizational structure, offered compelling decision-making perspectives.

As a public, four-year university, Tribal State University (pseudonym) links much of its educational history back to connections with a specific Native American tribe before officially becoming a formal Normal School in the early $20^{\text {th }}$ century. Its evolution from a small teacher's college into the fourth-largest institution in their state along with its recent growth and establishment of satellite campuses suggest an intriguing enrollment management environment - particularly as they weigh their recruitment goals against tribal and community connections. As a unit within their division of academic affairs, their enrollment management leaders faced imposing issues in their decisionmaking process.

The final study site location, Mountain View University (pseudonym), or MVU, also maintains connections to Native American populations. In addition to many of the standard units contained in enrollment management areas, they also oversee an office devoted to academic preparation for Native American students. Considering how the campus appears to embrace their location and connection to the community, their 
enrollment management leaders revealed an established strategy toward their decisionmaking practices that uniquely match their institutional mission.

\section{Study Participants}

Each of the six host site institutions had been determined to meet some variation of the four previously mentioned models of enrollment management: 1) committee; 2) coordinator; 3) enrollment management matrix; or 4) division of enrollment management. Through information obtained through the SACRAO and related websites, as well as each institution's website, enrollment management leaders were contacted via email to request their participation in this study. Upon clearance from the institution's institutional review board (IRB), invited participants were given consent forms explaining the study while acknowledging their rights to withdraw from the study at any time. An example of the consent form (and related IRB documents) appears in Appendix D.

The study sites, as well as participants, were purposefully chosen. Patton (2002) suggested the use of purposeful sampling in qualitative inquiries when the prospective participants are believed to yield rich amounts of revealing information concerning the subject of interest. Desired participants were the primary enrollment manager or leader (and, therefore, key decision-maker for enrollment management goals) for their institution, overseeing multiple offices/units. Each participant worked a minimum of five years in their current role or at a similar enrollment management-related position at their current or previous institution. The exact title of each manager's position was not pertinent, but the participants' titles all contained either "enrollment" or "enrollment management." 


\section{Data Collection}

As is appropriate and important for a qualitative inquiry, this study used multiple methods of data collection that included one-on-one interviews, documents and artifacts, and observations made during site visitations. How each was applied in this study will be explained contextually below.

\section{Interviews}

An interview guide (Patton, 2002) featuring open-ended questions was developed for use during the one-on-one interviews with the enrollment management participants. As the intent was to explore their realities and reveal the world through their eyes, the guide served to help construct the conversation rather than to limit it to a rigid set list of questions. The guide allowed and encouraged probing as part of a subject-driven dialogue between the participant and me.

An additional technique used to garner responses from participants was the use of a word association exercise. Carl Jung, the Swiss psychologist, suggested the possibility of connections between words and complex perspectives associated with the mental image and unconscious psyche drawn from word exchanges (Bachino, 2009). A series of enrollment management, higher education, and/or decision-making related contextual words was read to the participants in an attempt to ascertain potential patterns or themes among the respondents. A list of the words and responses is presented as Table 3 in Chapter five.

\section{Documents and Artifacts}

In addition to the interviews, information was acquired through documents and artifacts gathered at or about each study site. Examples include institutional reports, 
strategic plans, organizational charts, websites, recruitment plans, enrollment and retention reports, mission and vision statements, annual reports, recruitment brochures (viewbooks), among others.

\section{Observations}

With permission of the participant and any additional administrator deemed necessary, I visited the offices of the EM as well as meeting spaces, subordinate offices, buildings, and other activities or encounters as opportunities made available. Fieldnotes were kept about all observations made at the study sites.

\section{Participant Protection}

The individual EMs were given pseudonyms in the presentation of the data to protect their identity. Additionally, after transcription of the interviews and during the coding phase, each participant was assigned a unique number to further guard against identification. Any physical notes, documents, artifacts and the like were kept separately from electronic documentation. Electronic information was recorded on a personal laptop that remained password-protected.

\section{Data Analysis}

When a researcher embarks upon the task of collecting and analyzing data through a qualitative perspective, s/he serves as the instrument. Patton (2002) acknowledges there is no absolute or "recipe" to transform the data into conclusive findings. Converting documents, artifacts, raw notes, interview transcriptions and the like coalesce into meaningful, emerging results that richly describe the experiences of the participants requiring skill and diligence. This synthesis between informational data and results may nevertheless greatly aid practitioners of higher education as leaders attempt to 
make sense and meaning out of the experiences of their world (Major \& Savin-Baden, 2010).

Upon completion of data gathering, an analysis of the interviews, field observations, examination of documents and artifacts commenced (Wolcott, 2009). To assist with the management of interview transcript and coding, MAXQDA was used as an organizational tool. Themes emerging from the various data sources were identified through what Emerson, Fretz, and Shaw described as pursuing members' meanings when coding the data (1995). These experiences of the participants were portrayed and described through my own interpretations using analytic techniques such as members' terms and descriptions, explanations and theories, and storytelling. I used these techniques when presenting the data as a means of providing a voice and view of his or her world. Assisting with the coding analysis and emersion into themes was the use of analytic statements which helped connect thematic developments with initial insights and fieldnotes to help answer, "What's going on with the data?" (p. 146). A narrative portrait was written to help encapsulate the experiences and perspectives of the enrollment manager as discovered through the analysis and triangulation of all data sources.

When listening and transcribing the interviews, I utilized Labov's Functional Model as referenced by Poindexter (2002). According to Poindexter, this model suggests presenting the data taken from interview responses as "distinct parts with unique functions" (p. 65). This technique encourages the act of shortening the sentence structure into pieces based on an assortment of evaluative labels. In other words, I broke down a potential complex series of sentences into more individualized units of data that had 
unique meanings. Rather than simply examine the responses, "it is the narrator's interpretation that makes the story salient" (p. 65).

Agosto (2002) developed and applied a framework for Herbert Simon's bounded rationality and satisficing concepts in her study of young people's decision-making abilities when searching the web. While her contextual applications do not apply to this study, her synthesis of the theory provided a useful lens when evaluating the data. Through Agosto's study as a guide, the decision-making data from this study was viewed and assessed through the potential constraints of time and cognitive ability, as suggested in bounded rationality theory. Time constraints were broken down into two subcategories of being "imposed" and "self-generated." Cognitive stresses upon decision-making ability were also discovered in her study in the form of "information overload" (p.22). Data collected through the various methods was analyzed to determine if evidence of time-constrained decisions were made as well as how information and data overload may have been experienced by these enrollment managers.

The application of satisficing in Agosto's (2002) study was reported through two behaviors, "reduction" and "termination" of options (p. 23). Individuals satisfice through reduction of the overwhelming list of choices for consideration. In Simon's satisficing theory, he proposes that individuals have a "stop rule" when an acceptable choice is finally recognized and accepted. Agosto offered her label and identification of "termination" to mean when a search for a satisfactory choice was reached. Agosto's format was also applied in this study as the collected data was reviewed for indications of decision-making search options being terminated by enrollment managers. 
Rowles' (2003) dissertation eloquently summarizes and applies the garbage can model into a higher education context, primarily through the three separate streams of problems, solutions, and participants and how they intermix with one another (as if in a garbage can) based on their "temporal proximity" (p. 19). Data from this study was analyzed through Rowles' depiction of the model to ascertain if enrollment management decisions reflected interplay of time and place-bound problems, solutions, and participants.

\section{Trustworthiness of the Study}

Guba and Lincoln (1985) suggest that qualitative research employ a series of techniques to ensure credibility, transferability, dependability, and confirmability. The authors suggest including a thorough list which would make the research support the aforementioned characteristics. A trustworthiness table is listed below to recognize the methods utilized to achieve appropriate evaluative credentials for this study.

Table 1. Trustworthiness Table

\begin{tabular}{|l|l|l|}
\hline \multicolumn{1}{|c|}{ Technique } & \multicolumn{1}{|c|}{ Results } & \multicolumn{1}{c|}{ Examples } \\
\hline $\begin{array}{l}\text { Prolonged engagement } \\
\text { (credibility) }\end{array}$ & $\begin{array}{l}\text { Develop current and new } \\
\text { relationships } \\
\text { Establish trust with participants } \\
\text { Gather diverse range of data } \\
\text { Ensure accuracy of recorded data }\end{array}$ & $\begin{array}{l}\text { Devote time/effort to establish } \\
\text { trust in relationship building } \\
\text { Gather wide scope of data at site } \\
\text { and from participants }\end{array}$ \\
\hline $\begin{array}{l}\text { Persistent observation } \\
\text { (credibility) }\end{array}$ & $\begin{array}{l}\text { Gather in-depth and accurate } \\
\text { data } \\
\text { Discriminate data between } \\
\text { relevant/nonrelevant } \\
\text { Identify inaccuracies in data }\end{array}$ & $\begin{array}{l}\text { Conduct intentional investigation } \\
\text { Vary observations and setting }\end{array}$ \\
\hline $\begin{array}{l}\text { Triangulation } \\
\text { (credibility) }\end{array}$ & $\begin{array}{l}\text { Verify and compare data sources } \\
\text { aollect data from multiple } \\
\text { sources (interviews, } \\
\text { observations, artifacts, } \\
\text { documents, websites, jottings) } \\
\text { Recognize what data is missing }\end{array}$ \\
\hline
\end{tabular}




\begin{tabular}{|l|l|l|}
$\begin{array}{l}\text { Referential adequacy } \\
\text { (credibility) }\end{array}$ & Provide a sample "slice of life" & $\begin{array}{l}\text { Examine institutional websites } \\
\text { Review mission statements, } \\
\text { strategic plans, procedural } \\
\text { manuals, recruitment brochures } \\
\text { (viewbooks) }\end{array}$ \\
\hline $\begin{array}{l}\text { Member checking } \\
\text { (creditbility) }\end{array}$ & $\begin{array}{l}\text { Evaluate accuracy of data with } \\
\text { participant(s) } \\
\text { Verify interpretations or } \\
\text { conclusions }\end{array}$ & $\begin{array}{l}\text { Informal checking of data } \\
\text { gathered/recorded after } \\
\text { conclusion of interview and of } \\
\text { site visit }\end{array}$ \\
\hline $\begin{array}{l}\text { Thick description } \\
\text { (transferability) }\end{array}$ & $\begin{array}{l}\text { Include data base for transfer } \\
\text { ability judgment } \\
\text { Provide an empathetic, vicarious } \\
\text { experience for reader }\end{array}$ & $\begin{array}{l}\text { Document detailed and thorough } \\
\text { descriptions relevant experiences } \\
\text { and meanings of the } \\
\text { members/participants }\end{array}$ \\
\hline $\begin{array}{l}\text { Purposive sampling } \\
\text { (dependability) }\end{array}$ & $\begin{array}{l}\text { Gather data to allow for } \\
\text { emergent design and results }\end{array}$ & $\begin{array}{l}\text { Target varied potential } \\
\text { participants to allow for broad } \\
\text { range of relevant information }\end{array}$ \\
\hline
\end{tabular}

\section{Implications for Research, Theory, and Practice}

\section{Research}

This qualitative study investigating the decision-making practice of enrollment managers should assist in expanding upon the apparent scarcity of research available on the topic. Although many sources offer descriptions and strategies associated with enrollment management as well as decision-making approaches, few studies bring them both together, especially in a qualitative design. This study may bring clarity to the decision-making realities experienced by EMs while trying to satisfy institutional enrollment expectations.

Theory

The research gathered in this study will also help inform organizational decisionmaking theories such as Simon's bounded rationality theory as well as March and Cohen's garbage can model and their applicability in enrollment management settings. Should organizations attempt to craft or mold their structure to adhere toward a specific 
decision-making method or model, the evidence put forth in this study may help enrollment management practitioners plan and adapt accordingly.

\section{Practice}

Despite the numerous tools available to assist enrollment planners in their recruitment and retention of students as well as the increased attention given to the practice of enrollment management by professional organizations and assorted affiliations, managing an institution's enrollment remains a complicated task. If it weren't, computer models would simply project target markets necessary for maximization for enrollment. The complexity of this environment necessitates direct human involvement to discriminate the key factors from nebulous ones, and this subjectivity and individuality must be intertwined with enrollment management decisionmaking.

As state institutions continue to receive decreasing funding levels by state legislatures and governing boards while private institutions also face economic challenges due to a weak economy, the decisions enrollment management leaders may ultimately and significantly impact their institution's goals. Gaining a greater understanding on how the decision-making progresses, and especially how well expectations match reality, will help improve EMs' ability to plan, improve, or modify existing decision-making practices.

\section{Summary}

Crotty (2003) describes the process for a researcher developing a study as the "plan of action" (p. 7). This chapter attempts to clarify the procedural methods I undertook while conducting this qualitative study through the epistemological view of 
social constructionism. A total of six institutions of higher learning were desired as host sites and yielded a broad range of experiences and perspectives from their enrollment managers in terms of their decision-making processes.

Seeking emerging themes emanating from various data elements including interviews, observations, document and artifact analysis, and other sources, I gathered varied data suitable for triangulation. Understanding that a thick, rich description and language adds to a more in-depth and accurate portrayal of the data, a narrative portrait was also created to help elucidate and convey participants' stories. The purpose of these approaches was to exhibit through immense detail rather than simply tell a story (Patton, 2002).

As this chapter offered the procedures and methodology associated for this study, Chapter Four will present observations made during site visits and participant demographics. The fifth chapter will reveal associated themes that were uncovered. After the presentation of data, Chapter Six will conclude the study by discussing the findings thorough theoretical analysis, present limitations, explore implications upon research, theory, and practice, and make final recommendations and conclusions. 


\section{CHAPTER IV}

\section{DESCRIPTION OF SITES AND PARTICIPANTS}

In this chapter, descriptions and observations of each participant and site location are provided as a mental scaffold for the reader before presenting findings and outcomes of data analysis through thematic elements as well as a narrative portrait in chapter five. Additionally, a listing of participant demographics is offered to further aid in the reader's mental framework. This data was collected through interviews and interactions with the enrollment management leaders of six institutions referenced in chapter three, observations of their work environment, and an analysis of documents, artifacts, and photographs.

In order to maintain the anonymity of the participants as well as the institutions of which they are employed, each site and participant was given a pseudonym. Observations were either documented on-site on a laptop computer or recorded orally into a digital recorder for later transcription.

\section{Southern Regional College}

The building in which the meeting took place was a brick-walled single story building with clean, brick-tiled waxed floors to complement the walls of the building. Visited during mid-morning, there were no students visible in the halls, and despite the lights being on in the various offices and if not for what appeared to be minimal staff 
stationed in the offices, visitors might have assumed the campus was closed or at least operated with minimal staff.

There did not appear to be a consistent format or theme for wall decorations as old oil paintings of scenic landscapes hung down on some walls while others contained plaques of scholarship recipients and other honorees. Bulletin boards hung from other walls dangling flyers suggesting students sign up for scholarships, enroll online, pay for tuition with an automatic payment plan, and posters sharing information about alcohol abuse. A single wall-hanging held the campus newspaper, and several copies remained in its hold.

The administrative offices included financial aid, enrollment management \& registration, testing, library services, and recruitment. Several campus administrators were housed there including the President and VPs for Academic Affairs, Enrollment Management/Registrar, Financial Affairs, Community Relations, Student Affairs, and the Dean of Students. The receptionist, who wore a sweatshirt adorned with the institutional logo, greeted me by name and directed me to the Registration Office where the participant would be located.

The registration area was a medium-sized open office area containing a long single reception desk manned by two staff members. Another single workstation desk area remained vacant, and a circular metallic table with four matching chairs also sat unused and appeared to be part of the reception area for students. Sitting on top of the table was a six-tier tray containing various forms and informational cards. Assorted chairs outlined the walls with end tables containing additional forms and informational materials related to the student's admission and enrollment needs. Only the hum of the 
air unit along with faint murmurs from side hallway offices could be heard. I asked for the participant and was pointed toward his office.

Upon walking into his office, I was greeted by the participant, "David," who was sitting at his desk and guiding the computer mouse while shifting his eyes between the computer screen and me. He sat at a single rectangular shaped wooden desk with various paperwork on the sides and a desktop computer to his right. The space immediately in front of him was clear as if he pushed the papers to the sides of his desk.

Although not in total disarray, the shelving areas contained a mixture of what appeared to be books, reports, binders, informational materials and personal items. A grouping of his diplomas neatly hung on the wall to his right, and large tower of CDs sat against the wall and shelving area behind him. Instrumental music played in the background. David offered me a chair and seemed fairly aloof. I asked to close the door for confidentiality purposes, and he allowed it with a somewhat carefree response that suggested he would not be saying anything private or personal. He reduced the volume of the music and casually reviewed the consent form before his appearance and demeanor suggested that he was ready to begin the meeting.

The manner in which some of the questions were asked and answered seemed fairly formal early in the process with particular concern for professionalism but gave way for a more casual discussion as the conversation took shape. However, I never quite felt the responses were completely revealing until the word association exchange. The word association exercise required an initial response - either a word or brief words based on his interpretation of the word offered to him, and David responded accordingly. However, toward the end of the exercise, he began laughing and said it just occurred to 
him that he was being overly negative in his answers. This seemed to indicate he was surprised at himself for the direction this exercise took him compared to his other responses.

After the exercise was completed, I asked a few remaining questions. Although he seemed to respond more quickly than prior to the word association, he still reverted to a more professional, polished, and perhaps graceful manner. At the conclusion of the interview, he walked me to the lobby area and showed me where students enroll (it was the long, single desk just outside of his office) as well as the recruitment office located down the hall. While offering only brief information about each, he admitted that due to the low amount of staff around that there was not much else to see.

\section{Sloan University}

I was instructed by "Debbie" that our meeting would take place on the second floor of her office building, but it appeared to be a traditional dormitory. Walking into the facility, a bulletin board containing description of offices rested on the wall. Arriving on the second floor, a sign hung above the desk read "Admissions." A staff member instructed me to proceed down the hall. At the end of the hall, I walked into the office that said "Office of Enrollment Management" and checked in with the administrative assistant. Debbie smiled as she walked me back to her office and immediately called attention to the chaos of her desk, stating this was unfortunately what it normally looked like. Her U-shaped desk arrangement consisted of a computer and a credenza toward the back. The desk and credenza contained stacks of papers, books, binders, and stacks of miscellaneous documents. 
Wearing a colored shirt which seemed representative of the school's colors, she seemed fairly casual in her demeanor but acted very cooperative throughout the conversation. She had prepared for my arrival by producing the requested documentation and artifacts consisting of enrollment reports, projections, recruitment information, university profiles, among others. Upon completion of the interview, she offered me a tour highlighting the areas she supervised. She complained about their congested hallway space as we were occasionally interrupted by staff members who needed to get into their offices. Walking further down the hall, she pointed out the Admissions Processing and Records area and some confined storage rooms. At the end of that particular hallway was the international admissions office, and Debbie informed me they only had one individual responsible for all international admissions related tasks.

We went to the fourth floor, and she claimed this area was the nicer office space which reflected only moderately upgraded contemporary designs. It contained their testing center and consisted of only two staff people - a secretary as well as a coordinator. There were various testing offices with banks of what appeared to be outdated computers, and storage rooms lined in the hallways.

Upon the end of the tour, we walked back to her office and talked further about our respective perceptions and interactions with students as well as the complications associated with student information systems. Throughout the encounter, Debbie seemed to be very willing to answer questions and offer as much time as needed. At the same time, however, she emanated a somewhat fatigued quality which may be attributable to the time of year (it was toward the end of the semester) or due to other unknown or 
mentioned factors that had impacted her. Nevertheless, she did not appear to be reticent in her opinions.

\section{University of the Prairie}

Upon walking into the student union which contained "Shawn's" office, I found school colors, expensive wall decorations showcasing smiling students, and other furnishings reflected a strong commitment to appearance. A welcoming entrance complete with a building map suggested this institution was consistent in their marketing efforts.

To the right was the Admissions/Recruitment offices, but Shawn's office was located on the left. I opened the door to his office area, and there was a flat-screen TV on the wall (which was painted in the school's colors) with a news station on the channel. There were very large framed photos of the campus that matched the theme from the hallway decorations and reiterated the collegiate feeling.

Shawn was running a little late, but when he arrived, he eagerly shook my hand. Shawn did not quite manage a full smile but definitely projected an air of confidence as he directed me into his office. A significant amount of personal items, which also represented the institution, were also presented on the walls and desk. His desk credenza contained a small flat-screen TV on his desk projecting the local news. The lighting was very soft. Wide windows were on the far wall, and that coupled with the lighting projected a relaxed and comfortable setting that seemed more business-like in its décor as opposed to a traditional higher education administrator's office. Shawn's desk wasn’t particularly large, but it looked fairly expensive and fairly new. He invited me to sit at a somewhat oval conference table surrounded by armed, contemporary chairs. 
He was welcoming and friendly; however, I gained a sense that his time was going to be considered valuable. In fact, he reminded me that he had another appointment just under an hour from when we were supposed to meet. His responses appeared truthful, and he hardly hesitated on his replies to questions. A couple of times he did say, "To be honest with you," or "To be open about this." I reassured him about his anonymity, but he seemed somewhat guarded in his revealing of too many recruiting "secrets."

Confident but not cocky, Shawn's attitude and demeanor seemed to reflect his perception of the type of EM to lead this institution. We finished in just under an hour, and he prepared beforehand the paperwork I asked him to complete, but he did not offer any additional documents to take with me. In fact, he showed me some things but said, "I'm not going to give these to you." There was no time for me to take a guided tour, so I walked around to familiarize myself with the surroundings. His administrative assistant came in 5 minutes before we ended to inform him his next appointment was already there.

\section{Garden State College}

The building which contained "Mark's" office contained many enrollment management-related offices in its two floors. First floor areas included advisement, enrollment management, veterans affairs, special services, a student success center, a student welcome center while second floor offices included financial aid, student support services, student life, student affairs, and the testing center.

Mark's office was located in the advisement area. The waiting area is quiet and visibly devoid of students. Beyond the seating area was a reception station staffed by two 
individuals who greeted me with a smile. As I explained my appointment with Mark, he stepped about halfway out of his office and waved me on back to his office - the final office at the end of the center.

After shaking hands, I was immediately taken by the lack of clutter on his desk as well as the overall cleanliness of his office area. The office contained a rectangular desk with a desktop computer sitting directly in front of the chair while virtually no papers appeared on the top of his desk workspace. He invited me to sit on the couch toward the far end of his office (but also facing his desk). The couch was flanked on one end by a more formal sitting chair upon which he took a seat.

Mark was extremely quiet in his speaking tone and moved at a slow but deliberate pace when walking as well as speaking. Even hand gestures did not seem to be wasted. Although he was reassured before and during the interview that his anonymity would be protected, he openly admitted withholding names and viewpoints concerning some of his co-workers and/or supervisors. He even informed me with a reassuring, "I trust you" when asked about recording the conversation, but he refused to offer true opinions, feelings, or beliefs on certain subjects.

Mark and I have worked together in prior professional situations, and despite some of his self-censorship, he seemed to truly wish to participate in the interview and be a part of the study, perhaps out of consideration for me given our current professional relationship.

Mark had strong views of what motivated and propelled him while working in enrollment management. While perhaps not entirely satisfied with his station as an enrollment manager at his institution, his commitment toward assisting students and 
working with faculty seemed to propel his passion for enrollment management and impacts the types of decisions he makes.

At the conclusion of the interview, I asked to take pictures of his office and area. While agreeing, he also offered a comment suggesting he is "OCD" (obsessive/compulsive disorder) when it comes to how he has office organized. The lack of clutter and overall organization of the office appeared to back up those claims, whether in jest or not. He did not, however, have the participant basic demographic form completed prior to arrival because he was "too busy" to complete it.

\section{Tributary State University}

The participant, "Doug," informed me of his office location in a building which housed primarily enrollment management functions. Upon walking into the building at the designated time, Doug was just inside the door ready to greet me. The second floor housed the recruitment, admissions, veterans affairs, records areas along with the leadership of those areas. The office environment contained a mixture of open topped (offices with walls that did not quite reach the ceiling) single-occupant offices, a small waiting and sitting area, and counters for the admissions and records areas.

If visitors were unaccustomed to this type of educational environment, it would be reasonable to assume s/he might be confused about where to go. This was confirmed by Doug who voiced concerns over various entry points for visitors as well as a lack of a greeter - someone to walk or initially direct the student on where to go.

When we arrived at his office, he offered me coffee before sitting back in his recliner behind his desk. Two comfortable arm-rest chairs faced his desk. His desk was 
neatly organized despite the four piles of neatly stacked papers. The desk (as well as the credenza located near the office door) seemed clear of any dust or dirt.

Although not friends outside of work, from time to time Doug and I rely on one another for professional advice, commentary, or thoughts. However, his responses seemed to be presented in a professional and possibly practiced style; he has always presented himself in such a manner.

The interview lasted nearly thirty minutes longer than expected. At the conclusion of the interview, he walked me around the office space. He mentioned repeated concerns over students having to visit multiple office spaces and/or counters before finally getting the answer or help they needed.

The décor seemed representative of the colors of the institution, and pennants, pictures, photographs, jerseys, etc., adorned walls and office space. Few students appeared to be walking within the building, but there was ample activity by various staff. One of the staff Doug introduced me to commented how easily conversations can be heard given that some of the office walls do not go all the way to the ceiling. I could openly hear discussions being taken place among the records area-mainly coworkers talking to coworkers.

Doug devoted ample time for the interview as well as the general tour of the office space and surroundings. Additional staff offered friendly and welcoming greetings suggesting the environment may be one of a collegial and shared nature. Considering the openness of their office environment, it may have been difficult to have otherwise. Even those who have doors on their offices did not keep them closed.

Mountain View University 
The 3-story building which housed "Rachel's" office represented a more traditional collegiate design. Upon entering the building, it seemed as if time had stood still in terms of decorations and design of this building. It appeared "cold" as the walls were white or a faded gray and made for a dreary first impression. I made my way up the stairs, and each floor seemed to have the same design and layout as the next.

I arrived at the third floor and found Rachel's office which had her name and title on the glass door. When I looked into it, I could see a vacant reception area with a desk that contained no nameplate but various stacks of paperwork. Post-it notes and promotional materials were on the sides of the desk. I opened the door to the office and heard a voice say "Hello?" Rachel came around the corner and smiled graciously as she walked me back to her office just beyond the reception area. This entire office area consisted only of two offices - the vacant office (which I was told later by Rachel was manned only by students) and her office itself.

Her office held some personal items but appeared to lack the branding of the overall institution. Stacks of paper surrounded her computer on her desk with binders and folders surrounding it on the floor. She advised me to sit at her circular to conduct the interview. This institution's semester had not started yet, and even though there was a large window on one wall in her office, I did not see a lot of activity going on outside on the campus grounds below.

Rachel's office seemed peaceful at the time although the paperwork and binders located around her desk suggested she remained busy. She was very communicative and engaging in terms of looking me in the eye during our discussion. I felt she was very open and honest, so it made the interview flow more like a conversation. Rachel gave me 
a detailed tour after we concluded the interview beginning with hardhats for us both, indicating we were going to eventually walk into an area of construction. She mentioned how most of her offices that she supervised were located in that particular building and that some of the signage was incorrect. Some offices were vacated yet still had previous signage on them.

We went to the learning resource center which was very cramped, and their spatial limitations were quite evident. She made a comment that one of their testing areas was the breezeway to their building which had no privacy whatsoever. This area would soon be moving to a renovated area which would also house the success center including academic advisors.

She showed me the new office suite (which contained meeting and testing rooms, computer labs, and individual offices for advisors) with great pride and relief to move away from the current cramped areas. After that, she walked me back to her building, which was curious considering she didn't take me to those offices first. It was only upon our return to the administration building where she showed me the admissions, registrar, veterans, financial aid offices. I sensed that she was appreciative for the opportunity to tell her story and showcase the new directions her campus would take for those enrollment management areas.

\section{Participant Demographics}

Analogous to the enrollment management-related titles they held, each participant possessed both similarities and differences. A list of demographical information is presented below in Table 2 to help the reader gain a contextual perspective on these leaders. 
Table 2. Enrollment Manager Characteristics

EMs in this study have attained various degree levels

One achieved a doctorate, another is in progress

All have a master's degree

Two achieved their associate's degree before obtaining their bachelor's

EMs in this study have varied positions before working as an enrollment management leader

One worked entirely as a faculty member

One worked entirely in student affairs

Most worked as director of a related enrollment management unit

All were currently employed by their current institution before promoted to current role

EMs in this study have "enrollment management" in their title, but at various

organizational levels

Two are Vice Presidents

One is listed as Executive Director

One is listed as a Dean

Two are listed as Associate Vice Presidents

EMs in this study have different reporting structures

Three report directly to their institutional president

One reports currently to a vice president in one division before switching to another in upcoming months

Two report directly to the Vice President for Student Affairs 
Understanding some of the basic organizational and educational background of the participants should provide a fundamental perspective before reviewing the findings that will be presented in chapter 5 . 


\section{CHAPTER V}

\section{FINDINGS}

Against the backdrop of the site and participant descriptions presented in chapter four, this chapter presents themes discovered through careful exploration into the data to reveal the participants' voices and realities. All data gathered (as detailed in chapter three) was openly coded (Wolcott, 2009) with assistance from the MAXQDA tool. As this study is grounded in social constructionism, the coding effort was conducted to illustrate and share the participants' realities and experiences. Revealing the varied realities of each enrollment manager as it related to their decision-making was essential to provide the reader their unique experiences as well as insight into how they applied meaning to their perspectives (Emerson, Fretz, \& Shaw, 1995).

The participants' experiences will be portrayed and described through my personal lens using analytic techniques such as members' terms and descriptions, explanations and theories, and storytelling. I used these techniques when presenting the data as a means of providing a voice and view the participants' world. Assisting with the coding analysis and immersion into themes will be the use of analytic statements which help connect thematic developments with initial insights and fieldnotes.

The results of a word association exercise is provided toward the end of the chapter before a concluding narrative portrait representing the lived experiences of 
enrollment managers who participated in this study is offered. The intent of the portrait is to allow the reader the opportunity to gain perspective and meaning for the findings presented in this chapter against the backdrop of the context of the enrollment managers in this study as described in chapter 4. An extended discussion of findings will occur in chapter five, which will incorporate themes with the research questions and theoretical perspective.

\section{Thematic Findings}

A number of themes arose from the data that help to illuminate the lived experiences of enrollment managers as they navigate within their decision-making environments. These themes were identified through a holistic review of all data sources and subsequent qualitative analysis as described in chapter three.

Major themes were identified within the interview data and are presented by sub header in the sections that follow. Theme labels are derived either from collective experiences or a direct quote from a data source that captured the meaning of these perspectives, events, or beliefs.

\section{Every day varies}

Some EMs had a difficult time describing a typical day. Most expressed their days were full of various meetings, and one claimed his day was "just a series of meetings." One described a considerable portion of his day was "putting out fires." As one participant said,

But it, there's no real day-to-day. I have an ongoing list of things that I need to get done, and I usually try to schedule, I'm going to work on this and I'm going to 
work on this, and these are the priorities. And sometimes I get to that, and sometimes I don't.

\section{There's not enough time}

A common theme among all of the participants in this study was a feeling of not having enough time to achieve their goals. One voiced concern how her enrollment management area keeps adding programs and projects while "never take anything away." This participant elaborated, "People in general have an increasing desire for immediacy." Some participants expressed their preference for taking time to make decisions and responding or acting with more confidence knowing it was the "right" decision. One participant stated that she has grown used to "working with disruption" and claimed she often has to stop the planned activities or projects for the day and react, calling this issue "stopping and reacting." Another stated,

I think we're real good about solving a problem. We're not real good from a cooperative standpoint of 30 days later stepping back and saying, ok, let's look at these situations and say, what got us there, how do we fix what got us here, and continue to fix what we see in front of us.

Some EMs labeled interruptions as dealing with the "minutia of little things," as if it were a hindrance to what they considered their priority (and preference) of planning and dissecting problems. As one participant said,

And then, I get very little think time, is what I'm going to say. I spend a lot of time reacting to requests from administrators, faculty, whomever about how we can help them, what we can do, which leaves not as much time for actual planning as I would like. 


\section{Enrollment management is a competitive environment}

Competitiveness was a theme found through the data and expressed by many EMs in this study. While some mentioned this competitiveness originated from the need to recruit students, others reflected with surprise at how EMs appears to be less helpful to one another compared to other areas on campuses. One participant shared her story, I found it very different than when I worked in student affairs. In student affairs, if somebody [from another institution] called and said 'what are you doing on this?' You would copy everything that you have, send 14 digital files and say 'take whatever you need that makes your world better.' If you ask, 'how is that you all are thinking about your financial aid leveraging around [state funding source],' you're going to hear cricket, cricket, cricket, because that would be considered proprietary...

One EM was so protective of his enrollment data that he refused to offer any samples of his reports outside of his office during the interview. This same EM confessed to being a competitive person by nature and how being tasked to recruit new students suited him, “...but you're always on stage. That's byproduct of the job.”

\section{Data is critical when making decisions}

In nearly every meeting with an enrollment manager, large binders, stacks of reports, surveys, inventories, budgets, graphs, and charts resided on credenzas, shelves, or piled on top of desks. Each participant referred to these institutional, regional, or national sources as containing valuable data including enrollment-related statistics or projections. Some spoke very passionately about the particular kinds of data desired for decisionmaking and the need for increasing enrollment or, as one participant stated, the "focus on 
the numbers," may be the motivation for this value placed on collecting and reviewing data.

The apparent attachment and reliance toward getting and using data by enrollment managers was prominent among the participants. Despite this dependence, one EM did caution, "Data is only as good as how you plan to use it."

\section{Good, usable data can be difficult to get}

While all participants stated they use data to make decisions, few of them stated they personally generate the data needed. Most relied on either an employee within their operational unit or their Office of Institutional Research to produce desired reports. Some EMs seemed to be frustrated with their student information system in terms of being too complicated for their already understaffed employees to generate reports.

Gaining access to information may not be the problem for some, but getting usable, correct, and timely information was. One participant stated, “. . a all of us I guess have a tremendous amount of data if we can just (laughs) get it out in the form that we need it!" Another participant lamented over the lack of resources to analyze the data and stated,

So, in my opinion, we don't have the resources to work through the existing data we have to collect, to compile, to synthesize, to aggregate into usable numbers to help us make decisions. So, data is too little, too late in my opinion.

Apparent frustration over trusting the information produced from these reports was expressed by many EMs. One stated, "Since I have come into this position, the one thing that causes, well, eats up a lot of my time and causes me great heartburn is quality data." Participants who were responsible for branch campuses seemed to echo this 
frustration as multiple campuses meant additional opportunities for error or miscalculation, and one stated, "My fear is sometimes we send stuff out that's $90 \%$ accurate."

\section{Sometimes, you make decisions on instinct and experience}

Although all participants preferred data for making decisions, several EMs

admitted they also made decisions by trusting their own intuition or previous experiences. On occasion, data and experience may collide as one EM stated, "I won't say that I go against or would go against data, but there are certain things that I feel strongly about because I know they worked."

One EM's response offered a possible contradictory perspective by stating, I am absolutely convinced that one should not make decisions based on whim or anecdote. I like to see that there is some validity to the thought process, and it's not just emotion or whim. I also make decisions within the knowledge of the culture and personalities and what's acceptable to the institution.

Some EMs offered an almost apologetic admittance when they stated they use instinct to make decisions while others seemed to accept it as a reality. One EM said, I guess I rely heavily on my gut. (laughs) I know that's probably not the most formal way to make decisions but it has steered me correctly for many years, so I listen to it a lot.

\section{Reactive vs. proactive decision-making}

Another common theme was the tendency to make reactive decisions as opposed to deliberate and intentional decision-making practices. Rather than pensively crafting a decision based on confident expectations of results, some EMs tended to react by 
attempting multiple solutions in the hopes one may address the issue. One EM likened decisions to a shotgun, "You just hope that you hit something ... we just throw out a whole bunch of things sometimes..."

Whether having to respond to each day's trivial matters or be forced to respond to issues, projects, or difficulties imposed upon them by a supervisor, EMs were forced out of necessity to forego planning and preparations in their decision-making. One of the participants elaborated,

We need to be proactive versus reactive. We're not seeing the fly balls popped up in the air. We've got to be able to catch those versus just assume that everything is running fine.

\section{We must have strategic planning}

Many of the participants suggested the importance of having strategic plans in place to guide decisions. One EM's statement captured this theme well:

The institution should have a plan, and you should be making decisions that are aligned, I've seen from time to time, not necessarily here, but I had seen institutions where you have a very disjointed planning process. Everyone's doing their own thing, and there's no holistic direction from the institution. So, there should be an enrollment manager is implementing his or her plan, there should be a bigger plan in play with that linked to it.

\section{"Everything comes back to the numbers"}

Another prevalent theme was the focus on recruiting new students to ensure enrollment increases. One participant was especially emphatic with his assertive proclamation of, “...you can’t ever lose focus of the numbers.” This same participant 
shared the feeling of pressure that comes with the expectation to meet enrollment goals each year by stating,

Enrollment management is twenty-four/seven, three-sixty-five pressure cooker. Because it's a lot like football coaching. You're only as good as last season, and there's always the need for more wins.

Most enrollment managers in this study expressed a concern over meeting enrollment projections, and promotional materials, pamphlets, booklets, and even office décor reflected this theme of efforts to attract students to their campuses. One EM offered this statement about the recruiting students,

It's just fascinating to me that year after year it's well, you need to be doing this, well now you need to be doing this, and at the same time nothing's coming off the plate. I mean, we're still sending letters, right? That was the way it used to be done, was we sent letters. Well, we're still sending letters, now we're sending emails and we're sending texts and we have social media sites, and we have our internal portals, and all of these things we're doing, and nothing ever seems to go away...

\section{"Who's screaming the loudest"}

Enrollment managers listed a variety of influencers that impact or direct their decision-making processes. Some listed co-workers or their staff; others listed staff all the way through their president up to their governing board. One participant articulated, "Well, if the president wants it, that's first (laughs). Um, if my vice president wants it, that moves up on the priority list." Another EM was more direct in his response, "You 
know, I have two jobs. First is to take care of the students, and the second is to make our president and anybody I report to look good. And it's hard to do both sometimes..."

When asked about the various influencers to help direct prioritization of projects, one participant replied, "Who's screaming the loudest." This phrase was a common sentiment among participants who iterated the notion that some decisions that get made come from their superiors as a "have to" decision.

\section{Consultants aren't the answer}

Many EMs in this study relayed their experiences and/or perceptions concerning the use of consultants related to enrollment management. Some had experience using the same prominently known firm and offered negative comments related to the outcomes.

One participant had this to say about his experience,

I always feel like they're not really listening. I think that they do some great work. Honestly I think that they're able to put a lot of information in and spit out what should come out of it. I don't think that they're realistic in thinking about our clientele . . . from a statewide perspective they don't get . . f first-generation college students, I don't think they get adult students . . . they're very myopic in [thinking] if that student is going to come to you, you can figure out exactly how much money to give 'em, and that's going to make their decision.

Another offered a similar opinion of the return on investment, People like [name of firm], and others, that's their business. They, if there's not an ... emergency on your campus, they need to create one. Otherwise, why do you need them? They're very, very expensive. To me, you're being much better off bringing in someone you can trust. Someone that doesn't have to create an 
emergency that's going to give you an objective opinion and there aren't many of those people.

\section{Faculty need to be our partners}

Several participants spoke of their desire to include faculty in enrollment management strategic decision-making but voiced few successful examples. One stated, "If you are really looking at how you can grow enrollment, you're going to have to get into the academic business." Some seemed to voice concern regarding the lack of authority or control to make faculty recognize the importance of aligning enrollment goals as one participant expressed, “They just don't like being told what to do.” Another EM said, "I get a little frustrated with scheduling because we have developed a culture [of] scheduling . . . for the needs of the faculty not for the needs of the student."

One participant voiced his concerns over the faculty's lack of knowledge of enrollment management fundamentals as well as overall communication between both areas,

...but when I talk to the faculty, they still have no idea of what we do. And, but I've talked to a lot of our people about the faculty, and they have no idea what [the faculty] do. And so, we're trying to help the student but we all don't get the big picture.

\section{Enrollment expectations aren't always realistic}

The theme of enrollment expectations flowed both into recruitment of new as well as returning students. While some participants expressed feelings that their campus leaders' incoming enrollment expectations were somewhat realistic, the opposite 
appeared true for retention of current students, calling it a "complex" issue. Some also expressed the desire for immediate results, and one EM commented,

I mean it takes time for that, to see results. I mean, when a student starts . . they don't go into your success bucket until four years later ... or six years later, and then there's the whole philosophical question: Is it really realistic to expect a student these days to start at one institution and stay there and finish in four years? Probably not.

One EM discussed the expectation of predicting enrollment and related his experience, “...the easiest prediction to give is 'break even,' because what you want to do is under promise and over deliver. Your own worst enemy is huge success." Another participant voiced her thoughts on pressure from the entire campus when she stated, I think it's just hard. I think this is hard work. It is not all recruitment and fun, you know, it is making big decisions about the university with lots of people counting on you to make it work and not always having realistic expectations about what that could mean.

\section{We have good employees, but there are not enough of them}

A reoccurring theme among participants was a strong reliance and supportive view of their respective staffs, not just in their individual work but also in overall planning and incorporation into EM decision-making. One EM voiced this view of her trust and faith of her staff,

I think my people are fantastic. I think they do great work, and I think they work very well together. You know, I couldn't be any happier with my direct reports. 
[I] would fight to the death to keep them together even if I wasn't their leader - I think they are that strong a working unit.

Part of EM's vocal backing of their staff members related to their perceptions of the amount of overload certain staff members shoulder. One EM shared, “...it's been about six key people working every weekend, eight to ten hours on the weekend, extra hours every day to keep up with the Records job and trying to do this." Later, this same EM stated, "I'd say we have enough money, not people. Don't have enough people, don't have enough time."

EMs in this study seemed to commiserate with the challenges and demands of their staff as well as being powerless to make decisions to alter undesirable situations. One participant stated the following concerning his financial aid office,

They're good people. There's a lot of tenure, there's a lot of skills. It's just a set of rules that are totally beyond my ability to affect. And, I feel badly for an office that I believe is overworked, that a lot of anger is vented at them from internal and external constituencies and have little ability to change it.

\section{Stable leadership is essential}

Nearly all EMs in this study related situations and perspectives on how executivelevel leadership has either positively or negatively impacted their enrollment management efforts. One participant shared his perspective regarding his institution's president who was nearing retirement, and the next level leadership were all simply "waiting for new blood and energy" before attempting new strategic directions. One EM felt the instability of having nearly a revolving door for both the resident and the provost during a seven-year span that stalled "all the momentum as a unit." 
For those who felt their institution had stable leadership, EMs felt their institution was moving into a positive direction. One participant described the collaborative nature of his president as opposed to a previous one, "I like the collaborative style. And she's been here two years, and I would say there's been a lot of energy and a lot of very positive steps."

\section{Unexpected Themes}

While preparing for this study, there were numerous themes I anticipated due to either my own experience or through reviewing the literature. In the following section, I will highlight some of the more prominent themes that took me by surprise in terms of their blatant appearance through data analysis or their potential importance toward revealing the lived experiences of enrollment managers in this study.

\section{Reluctance to Share Information}

While not a dominant theme, some enrollment managers relayed their disappointment with their willingness of fellow enrollment managers to assist colleagues with best practice initiatives in their state. One EM in particular cited her background in student affairs and how the leaders in that field were extremely willing to share philosophies, strategies, and even detailed plans if it meant helping out a colleague. In her experience with enrollment management, she was highly disheartened to see a more guarded stance toward protecting plans and effective strategies when the sharing of that information might assist other institutions in increasing retention and graduation rates.

This reflects the competitive environment of enrolling students (which was an anticipated theme), but in my experience in enrollment management-related associations, I perceived an open and collegial approach toward solving problems. However, the bulk 
of my experience involves a specific non-recruitment based unit within enrollment management rather than the field from a more holistic approach, and that could be the participants' point of view of which she was referring. Regardless, her perception gained weight in this study as another EM in this study refused to offer any reports or artifacts to me for possible fear that his "recruiting secrets" may be revealed.

\section{Potential for Isolation}

During the interview process with the EMs, I was surprised by their candid expression concerning their opinionated views and feelings associated with aspects of serving as an enrollment manager. All participants offered factual responses, especially to early questioning, but as the interview turned more conversational, answers seemed more subjective and personal. Although not universally applied among all participants, most EMs seemed to demonstrate relief as they shared their frustrations with me.

As stated previously, I had existing professional relationships with nearly half of the participants, and their responses were expected to possibly be more subjective and open. However, even with individuals who I had never personally met prior to the interview encounter but knew I possessed a background in enrollment management, they appeared to exhibit a sense of liberation to have a colleague hear their irritations, frustrations, and passion. This may be encapsulated through the comments of one participant who told me after the interview concluded that it was "nice visiting with someone who gets it."

\section{Value on Good Employees}

Although surrounding an EM with quality, hard-working staff was discussed as a repeated theme among participants, the sheer value placed upon hiring the "right" staff 
member versus the "wrong" individual was revealed during a particular line of questioning during the interviews. Each participant was asked to offer examples of what they would consider "good" and "poor" decisions. In nearly every interview, enrollment managers relayed their hiring experiences, citing specific examples in at least one or both categories. Although I expected enrollment managers in this study to place a certain amount of worth concerning their staff, I was surprised by how much value, respect, and significance they placed on their individuals. Perhaps it was reflective of the limited resources available to hire additional staff or just an overwhelming appreciation for their abilities, but there appeared to be no question good employees meant a great deal to EMs. Finding the "right" kind of staff member did not just signify an ability to do one's job effectively, some EMs also signified that to mean the "right" staff member's personality to be able to mesh well with co-workers and additional colleagues. While some EMs listed "personnel issues" as one of the potential interruptions into his or her daily schedule, perhaps finding the "right" staff member meant s/he would face fewer disruptions in the workforce as much as it meant job performance. Both personality type and ability seemed to have relevance in an EM's perception of a quality staff, and that would ultimately impact their ability to make decisions.

\section{Our Students, Our Campus}

Although it is expected that enrollment management leaders know their campuses from an in-depth perspective, I did not expect such a pronounced and protective claim over the EM's type of student and campus as a whole. This theme seemed to be revealed mostly during the dialogue concerning the use of consultants. I was surprised at how many enrollment managers disregarded most of the recommendations supplied by 
consultants who were supposed to present individualized proposals catered to the needs of the student as well as the goals of the institution. However, these suggestions were not personalized enough for most EMs in this study.

Perhaps the sheer overwhelming cost of these services left the enrollment manager wanting more out of the time and financial commitment already given, but their declination of those suggestions may be more reflective of the connection an EM has with his or her college or university. I was able to review one institutional proposal (submitted by one of the participants) from one of the leading consultants in the industry, and there appeared to be a mixture of customized recommendations along with solicitations to use more of the organization's services (including planned site visits). This may suggest the EM's commitment to making an institutional focus in their decision-making efforts rather than commit to what a consultant suggests are best practices.

\section{The Effect of Multiple Campuses}

At least half of the EMs in this study referred to satellite campuses which complicated an already complex enrollment management environment. Although I was aware of some satellite or multiple campuses that certain EMs may either oversee or have some involvement, I was surprised to learn of how the additional enrollment managers who cited responsibilities with satellite or branch campuses. Some of these branch campuses appeared on the enrollment-related reports they viewed routinely, and some EMs mentioned how they needed to travel to some of these campuses from time to time. Considering the already stated interruptions to an enrollment manager's expected schedule, having to not only travel to other campuses for meetings and interactions but 
also monitor their respective application, admission, and enrollment trends added a layer of intricacy to an EM's list of responsibilities that I had not considered.

Surprisingly, those EMs did not cite their multiple-campus responsibilities as entirely separate from their main campus ones which may suggest they view it as merely one of the tasks of which they are accountable. Nevertheless, they still mentioned frustrations with communication and synchronization with those campuses as they would attempt to align strategic decision-making efforts. In retrospect, inquiring specifically about the potential for an EM to be involved with decision-making strategies at multiple campuses, rather than how it emanated naturally from interviews, could have aided further in this study's efforts to reveal an enrollment manager's environment.

\section{Absent Themes}

Just as themes appeared from the data that I did not anticipate, there were themes I expected to find but either did not or found in smaller amounts or in a differently meaningful context. The following is a listing of those themes I expected to discover through interviews and overall data analysis.

\section{New Students Remain the Priority}

Although retention and graduation of students was discussed during the interview dialogues, I anticipated a greater emphasis on behalf of the enrollment manager in terms of openly stating they were a priority. Even when stated they were a priority, the discussion and responses from the participants seemed to suggest the drive to get new students enrolled continued to outweigh the importance of retaining and graduating the students they recruit. In recent years, there has been a growing national focus toward keeping the students an institution recruits as well as a greater emphasis on graduating 
them, and the enrollment management literature suggests a strategic focus upon all three main categories.

EMs shared stories and perceptions of always being asked about "the numbers" with the main tool of measurement serving as the final enrollment tally for the semester. This evaluation of new student enrollment implied a de facto measurement of their overall efforts as an enrollment manager. There was no sharing of stories involving nightmares about their supervisor asking about retention or graduation numbers, thus further illustrating the EM's focus toward new student recruitment. I entered into the study expecting almost an equal concern for retention initiatives as well as an urge to graduate students in fewer semesters. Although they may very well serve as a priority for EMs, they did not appear to be on the same level as the new student focus.

\section{Not Buying What Consultants Are Selling}

As mentioned in previous sections, EMs in this study did not seem to uphold a tremendous amount of value on enrollment management consultants. Some EMs shared their possible annoyance with the manner in which consultants were brought on campus. One EM shared his displeasure with not being involved with the process (due to him being new to his position), and he ultimately felt their assessment was incomplete or at minimum, "too aspirational and not objective-driven" for him to embrace.

I anticipated a more open-armed response from EMs to consultants they may have used due my own experience with hearing others in my own work environment voice desires for their involvement to help with initiatives. As some of my colleagues have inquired with these organizations as to which campuses have utilized their services, some of the larger companies boast hundreds of institutions from all over the country with 
varying student demographics. This instilled upon me a perception and assumption that they most likely will have a favorable reputation. Considering the lack of financial resources that most EMs in this study mentioned as well as how costly enrollment management consulting services are, perhaps the EM's responses were of a statement of dissatisfaction with their deliverables. One EM felt these companies were very "myopic" in their view of what strategies would be effective for their respective campus rather than truly understand their campus' needs as well as the type of student who enrolls there.

\section{Little Talk on Diversity}

Much like the national trends concerning the focus on improving retention and graduation numbers, I also anticipated responses from EMs to reflect a concerted effort to address diversity issues as it relates to enrollment management. The rising population of certain multi-cultural ethnicities, such as Hispanic students, has been a popular topic in national publications, so I assumed I would obtain information on how EMs may strategize in their decision-making efforts to address these demographic shifts. Some of the campuses I visited contain high populations of Native-American students, and other than making casual references to them listed as a separate category on sample enrollment reports (which does reflect an institutional emphasis toward this population), there was little sharing of enrollment management plans for them.

As part of the demographic form, each EM was asked what subscription services used to gain insight or stay current on higher education or enrollment managementrelated topics, and only one participant listed a publication rooted in diversity issues. This surprised me as I felt at least some enrollment mangers, especially those with student bodies with established histories of enrolling students of multicultural 
backgrounds. In fairness, I did not ask an intentional diversity-related question hoping it may naturally evolve from the interview, and other than perhaps a cursory reference to a specific multicultural population as a recruitment target, there was little discussion concerning these students.

\section{Word Association Exercise Observations}

As discussed in chapter three, each enrollment manager was asked to participate in a word association exercise during the interview process. The words chosen were rooted in enrollment management, higher education, and/or decision-making and were used as an elicitor to assist the participant's own self-awareness and thereby generate richer responses. The process began with a word or short phrase to which the respondent echoed back the first thought (also a word or short phrase) that entered his or her mind. In Table 3 below, the ten elicitors along with each of the six participant's responses are listed.

Table 3. Word Association Exercise and Responses

\begin{tabular}{|l|l|l|l|l|}
\hline $\begin{array}{l}\text { Enrollment } \\
\text { Management }\end{array}$ & Decision-Making & $\begin{array}{l}\text { Campus-Wide } \\
\text { Teams }\end{array}$ & Recruitment & Retention \\
\hline Daunting & Churning stomach & A nice thought & Demanding & Very frustrating \\
\hline Numbers. Increase & Strategic & Challenging. Slow & $\begin{array}{l}\text { Critical } \\
\text { Collaborative }\end{array}$ & $\begin{array}{l}\text { Very important } \\
\text { Somewhat overlooked }\end{array}$ \\
\hline Misunderstood & Data & Fractured & Brand & Unsolvable \\
\hline Challenging & Could be better & Silos & Good & Poor \\
\hline Challenging & Thoughtful & Hit and miss & More difficult & Hard \\
\hline Enrollment. Broad & Contemplation & Complex & Competitive & Huge. Complex \\
\hline
\end{tabular}

\begin{tabular}{|l|l|l|l|l|}
\hline Graduation & Data & Resources & Time & $\begin{array}{l}\text { Enrollment } \\
\text { Expectations }\end{array}$ \\
\hline Joyful & $\begin{array}{l}\text { Critically } \\
\text { important }\end{array}$ & Short supply & $\begin{array}{l}\text { Only 24 hours in } \\
\text { a day }\end{array}$ & Instantaneous \\
\hline Critical & $\begin{array}{l}\text { Absolutely } \\
\text { imperative. Tool } \\
\text { for decisions }\end{array}$ & Adequate & $\begin{array}{l}\text { Challenging. I } \\
\text { go 100 mph all } \\
\text { the time. }\end{array}$ & Unreasonable \\
\hline
\end{tabular}




\begin{tabular}{|l|l|l|l|l|}
$\begin{array}{l}\text { (Participant wanted his } \\
\text { "smirk" as his } \\
\text { response) }\end{array}$ & Necessary & Limited & Little & Uncommunicated \\
\hline Rates are good & Under lock \& key & $\begin{array}{l}\text { Exceptional } \\
\text { financially. HR } \\
\text { not bad }\end{array}$ & Never enough & $\begin{array}{l}\text { Achievable with } \\
\text { strategic changes }\end{array}$ \\
\hline Out of our control & Infuriating & Limited. Scarce & Not enough & Outrageous \\
\hline Complex & Overwhelming & Good & Not enough & $\begin{array}{l}\text { Challenging. Always } \\
\text { be up 1 }\end{array}$ \\
\hline
\end{tabular}

Certain observations about the behaviors of participants during the exercise provided additional meaning and context for their responses. Applying this exercise toward the front of the interview appeared to prompt the participant into a more authentic and faster descent into their perceptions, beliefs, and experiences on the subject matter. Some participants followed directions exactly while others seemed to feel the need to justify every response with a detailed explanation as if to assure the credibility of their answers. When advised that the exercise was about to begin, most participants broke eye contact with me and locked eyes on particular focal point; some repositioned themselves in their chair perhaps as a means of preparing themselves for the unknown.

The word exercise appeared to work well in terms of generating a summative interpretation of the word or phrase offered to the participants. As shown in Table 3, most of the participants' responses could be categorized as negative. One participant was so surprised by the self-labeled "negative" responses he gave that he paused to call attention to this observation, “...I'm listening to myself as you're asking as you're giving me these phrases, and I'm feeling like I've got negativity..."

In one instance, another participant chose not to offer a response and merely smirked as if he did not wish to reveal his true thoughts. When I probed further, that participant asked to be recorded as "smirk." One participant used the word "complex" as 
her response for three out of the ten questions. Others used word "challenging" in multiple categories. These responses may suggest EMs view their environment as complicated and perplexing as the limits of their answers obligated them to encapsulate their perceptions into a shortened observation or definition of the elicitor. When taken collectively, responses such as "very frustrating," "demanding," "churning stomach," “fractured," "poor," "unreasonable," "daunting," "overwhelming," among others imply a pessimistic perception of those enrollment management elicitor words and phrases.

Although particular enrollment goals differ by institution, expectations placed upon enrollment managers to reach these objectives existed for every EM in this study. However, the perception for enrollment managers to reach their respective enrollment goals seemed to contrast between what they discussed during the interview compared to their immediate responses during the word association exercise. Most EMs stated their immediate supervisor offered reasonable expectations as to what could realistically be achieved during the next enrollment cycle. When attempting to forecast enrollment for an upcoming term, EMs would often share conservative numbers. One enrollment manager stated he would routinely "under promise and over deliver," and several EMs would predict they would come close to "breaking even" when compared to previous semesters.

However, during the exercise when all EM participants in this study were asked for a simple response to "enrollment expectations," nearly all of their replies were stated negatively. Answers such as “outrageous," “unreasonable," “challenging," "instantaneous," among others were given, suggesting that perhaps the expectations from 
the campus at large to sustain beating enrollment goals was not as reasonable as those shared by their direct supervisor.

The utilization of the word exercise seemed to provide additional context to the interview responses by participants. Not only did some of the participants vocalize their acknowledgement of how their responses could be construed, especially from a negative outlook, but the short answer responses also served as a barometer of sorts to compare more detailed response with a simpler, concise enveloping perception of the enrollment management-based words given as elicitors. The longer answers to interview questions combined with these shortened replies from the exercise aided in the effort to give voice and meaning to the participants' realities.

\section{Narrative Portrait}

In the following section, a narrative portrait will be presented to illustrate a potential segment of lived experiences representative of enrollment managers in this study. A narrative portrait helps illustrate some of the prominent themes observed and recognized in the lives and events experienced by the participants. These themes are brought together cohesively to help tell an overarching story and to drop the reader into a sample "slice" of the collective experiences of enrollment managers in this study (Emerson, Fretz, \& Shaw, 1995, p. 170). Although the name and the situations described below are fictional (a combination and representation of varying participant reports), the perspectives are derived directly from interaction with participants and the themes that emerged during data collection and coding.

\section{Taylor's story}


It was shortly after eight when Taylor opened his office door. He hurriedly hung up his messenger bag behind his door so he could wake up his computer from its digital nocturnal slumber. He paused briefly as he embraced the notion of "waking up" his computer with expectations that it will immediately be ready to assist him with what he needs. That's how he felt sometimes - as if others expected to flip a switch on his neck which would enable him to do exactly what others wanted. It's understandable, he recognized, as it was early August on a Monday, and the start of the fall semester was just weeks away. He knew his president would be anxious to know how enrollment was faring compared to the previous year.

This was a Monday morning ritual that Taylor knew all too well. A dark-haired man in his late forties, he wondered about his own potential enrollment. He debated pursuing a doctorate this past year, but the institution where he currently worked did not offer doctoral programs. Already feeling as if he had no time to keep up with the demands of his job as Dean of Enrollment Management, he questioned if could devote the necessary time and energy to achieve a terminal degree, especially at another institution. After working over ten years in the field of higher education, he was convinced this was his calling. Nevertheless, he could not think too long on it - his ambivalence would have to wait as the cabinet meeting would start in less than an hour, and he had to check to see if the enrollment reports have been generated by his registrar yet.

He opened the email program on his computer to see that 22 new emails had entered his inbox since he checked it last night from his home computer. Some were from students who had questions concerning their admission status, financial aid, or 
academic transcript. Others consisted of meeting invitations or reminders for later in the week. Those would have to wait, however, as Taylor was searching for an email from his registrar. He located and opened the email, then printed off the attached files. He needed this information to compare to last year's enrollment numbers.

The president, a leader of the institution for less than a full year, would surely be anxious to know how his incoming freshman and transfer numbers compared to past classes. Taylor had aspirations of advancing more programming efforts toward improving retention and graduation initiatives, but he was also very aware of the president's concern over public perception related to new student numbers. "This may be for the best, anyhow," Taylor thought to himself. Prior to the hiring of the new president, he had experienced numerous challenges and resistance from faculty who he felt did not either grasp or wish to be included in enrollment management efforts other than an occasional appearance during some of the larger high school campus tour programs. While he had good relationships with the deans and some faculty here and there, there seemed to be a disconnect partnering with faculty overall.

Getting faculty to show up at some recruitment event was one thing, but ensuring they are all attentive enough in their classes to take roll and notify the advisors if certain students haven't attended in a while, to say nothing of taking a more active, engaging role in connecting with their students which can improve retention was a different matter entirely. Taylor senses there still may be a feeling from some faculty (and perhaps a dean or two) that it's the student's sole responsibility to make sure they're successful and that faculty should not have such an active role in the student's persistence. Now may not be 
the best time to push that agenda, and honestly, he was just as anxious and stressed to know the enrollment figures as the president.

Reaching behind his desk to obtain a binder with various printouts and spreadsheets related to the previous fall term, he felt his pulse accelerate. Their campus switched to an entirely new student information system a couple of years ago, and there were still questions about how accurate the reports were in terms of generating definitive numbers for specific categories. His staff, already limited in number and resources, had worked late into the evenings and on weekends trying to convert data from the old system into the new one so that accurate reports could be generated.

Taylor previously informed the president of these challenges, but the reality that he may be sharing and providing figures with data that is less than $100 \%$ accurate turns his stomach. He briefly recalled a dream several weeks ago where he was being chased down a crowded street by a figure that resembled the president. In his hands was some sort of blunt instrument, perhaps a club. Although Taylor couldn't see with great detail as he was being chased, he felt that the president was gaining ground on him, all the time while yelling, "How many?!?" Taylor thought very little of the dream at the time, but it was mornings like this that made him realize how the concern over the numbers had crept into his subconscious.

He pushed aside a couple of stacks of paper made up of various "current" projects and tasks he's had to delay in favor of more pressing ones to create room for his comparison analysis. Before he could get too far into his task, his administrative assistant knocked on his door and informed him an upset student had arrived and demanded to speak to the VP concerning his financial aid. Despite the enrollment comparisons needed 
for the upcoming meeting, Taylor knew he had to meet with the student. On one hand, that's just the kind of person Taylor is - he genuinely wants to assist students, and that's why he came back to higher education after a brief stint working in sales. On the other hand, Taylor also knew full well that if this disgruntled student did not get a chance to meet with him right now, he would probably go straight to the president's office as this seems to be a pattern with many of today's students.

After the student left, not necessarily happy but certainly less angry, Taylor returned to the comparisons. As he made notations in the margins near the first-time freshman categories, he remembered a phone conversation he had with a sales representative from a well-known admissions and marketing firm. The rep was anxious to set up a webinar to explain how utilizing their products and services could increase the admission yield while expanding opportunities for new target markets for freshman. Although he left the door open for future opportunities, Taylor was cautious in his accepting of those claims. He knew Darren, a good friend and colleague working in a similar role at another institution, had an unpleasant experience with the consultant he chose. Darren felt they were only interested in selling their products and rehashed some of the strategies and recommendations used for many other clients, which did not translate well to "their" (Darren's) kind of student.

With the comparisons nearing completion, Taylor wondered how effective the summer overnight recruitment programs were in generating more applicants. Switching to an overnight event brought in larger amounts of prospects, but he wasn't certain whether or not those figures would turn out to be serious applicants, let alone enrollees. He pulled out a scratch piece of paper and wrote down, "Evaluate/measure summer 
programs" on it as a reminder to research this idea. While he did not possess the technical knowledge to create and develop a report that would identify the key variables to truly measure that program's success, he knew his registrar and support staff did. $\mathrm{He}$ also realized that this request would merely be added to the previous ones he had already given them.

Although he did not yet have data about the summer programs, he decided he would offer that for consideration to the president and other cabinet members. Whether up or down, there was always the question of "why." It takes considerable time to truly measure the impact of a program, let alone several enrollment management programs and initiatives, but he felt he had to give them, well, "something" as a reason. His gut told him those programs seemed to be effective based on the post-event surveys conducted. He couldn't always say, "We're working on evaluating our programming" each week.

Only five minutes remained before he needed to leave to head over to the administration building to attend the president's cabinet meeting and present his enrollment figures. "Increases in freshman applicants and enrollees, although somewhat minute, should make them happy," he thought. So far, Taylor had to admit to himself that the president hadn't been too critical of enrollment projections, but they were on the positive side of late. Still, he knew from dealing with the past president that there always seemed to be the expectation to be "up." For now, at least, he took solace that numbers were on the positive side.

Before heading out of his office, he took a few seconds to scan his electronic calendar to see what was to be done after his cabinet meeting. A lunch meeting with the housing director, three separate appointments with students, and an hour blocked out on 
his calendar to "Prepare for recruitment materials planning meeting" are what remain for this particular day. As he grabbed his cell phone, the enrollment comparison reports, his electronic tablet, and a notebook before heading out the door, he wondered what the odds were of keeping that schedule exactly as it was laid out - so organized on his computer calendar. Taylor chuckled to himself and thought, “I don't even want to think about giving a projection for that."

\section{Summary}

Chapter five presented the findings from data gathered as detailed in chapter three and against the backdrop of the enrollment environment described in chapter four. Upon the conclusion of interviews, document and artifact gathering, and site observations, all data sources were analyzed to begin formulating themes that emerged and coalesced. To assist in the coding process, the MAXQDA tool was utilized to help organize the data into chunks which aided me in arranging data points into specific thematic sections. Overall findings in the data were presented through themes. The administration of the word association exercise was presented along with specific reactions demonstrated by the participants. Finally, a narrative portrait integrating the combined experiences and perceptions was presented to assist the reader's understanding of enrollment managers by allowing them to view their situations through the lens of EMs.

Chapter six, the final chapter, revisits the research questions, and findings will be evaluated and presented through an analysis comprised through the literature and theoretical perspective. Limitations associated with this particular study as well as recommendations for further study will be presented. Contributions to research, theory, and practice will be discussed before a final collective summary and conclusion. 


\section{CHAPTER VI}

\section{DISCUSSION}

Chapter five contained the findings identified for this study and revealed the lived experiences of six enrollment managers through common themes demonstrating the participants' views, beliefs, and understandings related to their enrollment management environment. This chapter will present responses to the research questions and discuss the overall findings and themes through the lens and perspective of theory. Following will be limitations of the study, and significance of the study as it applies to research, theory, and practice. The chapter will conclude with recommendations for further research and a final summation of the entire study.

To help orient the reader and ensure a connection between the results and the initial inquiry associated with this study, the research questions are listed below.

\section{Research Questions}

This study endeavored to explore the factors that may contribute to or influence the decision-making process of enrollment managers working in two and four-year public colleges and universities located in the Midwestern United States. The study also inquired as to the efforts EMs use available data in their decision-making. These three research questions guided the investigative process:

1. How are strategic enrollment management decisions made on a campus that 
utilizes an enrollment management system?

2. As enrollment management professionals make decisions, what do they consider?

3. How do expectations relate to decision-making?

\section{Summary of Participant Descriptions and Findings}

EMs in this study possessed an assorted range of degree attainment and reached the director-level of a higher educational unit prior to leading their respective enrollment management areas. Reporting structure and titles varied among participants as some EMs reported directly to their president while others reported to various vice presidents.

Participants reported a high pressure environment, and whether their focus was on recruiting new students or retaining and graduating current ones, EMs seemed to agree that the enrollment expectations placed upon them were not always realistic. Reportedly repeating through the mind of an enrollment manager is the mantra, "It's all about the numbers."

Lack of sufficient or even adequate time was a very common theme shared by participants as EMs seemed to be impacted by "daily disruptions" which affected their daily routines and schedules. Enrollment managers in this study felt this dynamic and the unpredictable environment channeled them into making more reactive versus proactive decision-making. Most EMs greatly appreciated and trusted their staff members while sympathizing with how much they were "overworked."

When it came to decision-making, EMs were unanimous in their predilection toward accurate, quality data. Considering their inclination to primarily be focused on numbers, EMs placed a substantial amount of value upon data in their decision-making processes. However, they expressed frustration when data may be either untrustworthy 
or difficult to obtain. They seemed to somewhat reluctantly acknowledge the realities of having to use "instinct" or "gut feelings" to make decisions in the absence of data.

Perceptions toward utilizing professional consultants in the decision-making process varied somewhat, but most felt those organizations did not cater enough to “their" students. Regardless of quality data or consultant-based recommendations, EMs demonstrated a tendency to make decisions on "who's screaming the loudest." These "have to" types of demands included others besides their superiors-including fellow staff, faculty, and of course, students-but EMs relayed these interactions as factors that would disrupt or interfere with their current prioritization.

EMs also expressed a willingness for faculty to be involved in their strategic processes, but it appeared to be a challenge for most and there were few examples given of successful collaboration. An essential element to effective enrollment management decision-making, as expressed by EMs in this study, was stable and effective leadership. Coupled with the need for strategic planning that should govern the overall direction of the campus and from which all subsequent decisions should flow, enrollment managers relayed several examples of how both effective and poor leadership efforts had either propelled or stalled their momentum.

\section{Discussion of Findings}

In the following section, responses to the research questions guiding this study will be given citing examples from data analysis.

\section{Research Question 1}

How are strategic enrollment management decisions made on a campus that utilizes an enrollment management system? 
Enrollment managers in this study universally proclaimed data to be the primary desired factor when making their strategic decisions. Data took many forms, but the most cited were routinely generated application, admission, and enrollment reports, state and national trend analyses, credit hour production comparisons, academic proficiency of prospective and current students, retention and graduation rates, economic forecasts, student satisfaction surveys, policies and procedures, and publications associated with higher education and/or enrollment management.

A single "enrollment report" may be broken down into multiple sub-categories such as first-time freshman, first-time transfer, first-generation, total headcount, headcount by classification (i.e. freshman, sophomore), as well as if the institution possesses multiple campuses as well as special populations. The office space of most EMs reflected their dependence on data as numerous binders, reports, and additional information adorned their workspace. Possessing and gathering the data itself is only part of the responsibility for EMs as they are expected to take action and make decisions based on the data gathered.

Some EMs discussed the apparent dichotomy associated with needing timely, precise, and quality data compared to the realities of sometimes having incomplete, inaccurate, or even the inability to generate data due to resource constraints. Nevertheless, EMs in this study seemed to project a dependence on data to legitimize, validate, and/or guide their decisions. They also voiced additional limitations with data as one acknowledged that just possessing the data does not mean it "tells you what to do with it." However, even when they felt comfortable with the direction data insinuates, they were frustrated with the potential limitations (mentioning financial resources, 
staffing, legislative) to adequately address the problem. Another admitted that, although an enrollment manager may acquire all the data imaginable or desired, it was meaningless unless action was taken as a result. This ambivalence toward data reflected almost a "love/hate" relationship as some admitted their worries over quality data gave them heartburn or kept them up at night worrying about the accuracy of the next day's report. A review of some of the enrollment reports submitted to me corroborated these inconsistencies.

Aside from data serving as the most "ideal" for decision-making, EMs relied on what they described as their "intuition, "instinct," or "gut" to make decisions but also qualified it to mean these impulses were routed in their experience in higher education, enrollment management specifically, or private sector background. EMs had varied perceptions as to the effectiveness of making decisions this way as well as acknowledged their feelings of sometimes having no choice other than to make subjective decisions due to the lack of data informing them of the best solution.

However, some EMs openly admitted they may choose their own direction if they felt strongly about a given initiative despite what data (such as national or local trends) suggests. While the explanation for allowing intuition to dictate decisions over evidential data may be rooted in each EM's personal work experience, a greater justification could be the overall understanding the enrollment manager possesses of his or her campus. Many times throughout the interview (and validated through document and artifact analysis), EMs demonstrated an ownership of their institutional strategy based on "our" students or citing plans that work at "this campus." 
Those enrollment managers who claimed to have unstable leadership governing the campus (such as a soon-to-be retiring president, a newly hired one, or vast changes occurring at the provost and/or VP level) appeared to be more hesitant in their decisionmaking compared to those with solidified and established leaders. Those EMs experiencing an impending presidential transition felt as if most of the campus was in a type of leadership stasis and were reluctant to make any substantive directional decisions until the "new blood" had arrived. Stable leadership was not only an apparent prerequisite for taking action but also leaders who demonstrate the desire for collaboration and aligned efforts. This represents one of the proclaimed guiding points for campuses embodying an enrollment management philosophy, and it appeared the EMs in this study embraced this as a personal premise of which to make strategic decisions.

\section{Research Question 2}

As enrollment management professionals make decisions, what do they consider? Enrollment managers demonstrated a centered approach toward decision-making in terms of keeping the interests of their students and overall institutional culture at the forefront of their strategic decisions. One EM in particular stated that as a general rule, she first (rhetorically) asked what a potential impact a decision would have on her students and then asked the same for her staff. These questions seemed to permeate through most of the EMs in this study, and it also appeared to reflect a method of protection for each enrollment manager. It seemed as if they were conscious not only of decisions solving an impending problem or addressing a certain goal but also the 
resonating details that would inevitably impact the key focal point for them as enrollment managers: their students and staff.

This was another filter EMs utilized when dealing with professional enrollment management consultants who showcased either their previous successes at other institutions or presented national or regional trends to justify their recommendations. The EMs would process that information but ultimately ask the question, "How does this fit with our students/our campus?" This appeared to demonstrate a level of institutional commitment on behalf of enrollment managers who valued personal and truly individualized recommendations over an over-arching recruitment plan (for example) that may work for most institutions.

As protective as EMs were of their direct staffs and students, they also seemed to be very conscious of how decisions would impact their direct supervisor. At least two enrollment managers in this study openly stated how one of their primary jobs is to make the president "look good" while most at least acknowledged that if their direct supervisor wanted an issue to be addressed, the EM would move that expectation to the top of their prioritization list. One enrollment manager confessed the challenge between making decisions that he felt were assisting students compared to enhancing the president's image in that sometimes those were conflicting endeavors. Whether driven by a respect for authority, a direct charge laid upon them by their direct supervisor, or perhaps out of fear of repercussion, EMs seemed to be directly motivated in their decision-making by those in high executive positions.

Another prominent theme informing the factors involved with how EMs make decisions is the concern over enrollment numbers. While some were more vocal in this 
proclamation, all enrollment managers in this study agreed they focus a great deal of attention to the flow of enrollment numbers in their campus. Although retaining students was mentioned as a priority, the primary concern still seemed to be the push for acquiring new, incoming students. This conviction toward achieving enrollment increases places tremendous pressure upon EMs to break enrollment records and gain public relation recognition by boosting enrollment growth, especially when compared to peers or other institutions in the state. The pressure for enrolling new students could be considered a close companion to the notion of making decisions based on supervisor approval as EMs felt high enrollment numbers equated to making their supervisor "look good" while also fulfilling their expectations.

\section{Research Question 3}

How do expectations relate to decision-making?

Expectations placed on EMs in this study varied by source. Certainly, their supervisors articulated expectations, but they also felt a duty to respond to their direct reports in addition to any student and/or parental inquiry that may arise throughout any given day. Many EMs discussed the disparities of their day-to-day activities as these impromptu interruptions would disrupt their schedule. EMs felt obligated to stop their current project and address the issues bestowed upon them. The more immediate and apparent needs of supervisors, staff, students, and parents, the more the enrollment manager felt s/he must keep "putting things off” until sufficient time may be available. EMs seemed to be accustomed to these interruptions and varying intrusions upon their preferred timetable as they acknowledged this evident reality of serving as an enrollment 
manager, but they also seemed to quietly begrudge the interruptions as a constant barrier to achieving their more highly desired goals.

Regardless of pressure felt by their presidents, vice presidents, other highly placed administrators, or self-imposed stress, as well as the complexities associated with recruitment and retention initiatives and programs, EMs shared their realization that all of their efforts point toward a single measure-Is enrollment higher than last year — and that expectation for increased enrollment seems to be a momentous and meaningful facilitator for decision-making.

This next section of the chapter will consider the findings through theory as a lens for deepening understanding. As noted in previous chapters, theoretical perspectives suggested in Riley's (2007) dissertation helped inform me in selection of these lenses.

\section{Themes through Theory}

\section{Bounded Rationality}

Herbert Simon's theory of bounded rationality decision-making through satisficing conceptually reflects the notion that maximizing all available options for a decision is not realistic, and humans will therefore select a "good enough" decision to meet expectations (Lehman, Lyubomirsky, Monterosso, Schwartz, White, \& Ward, 2002, p. 1178). Agosto (2002) developed, applied, and operationalized a framework for this concept in her study of young people's decision-making abilities when searching the web. She identified examples of bounded rationality through time and cognitive constraints while recognizing elements of satisficing by the reduction of choices as well as a termination or "stop rule" of selecting the first acceptable option (p. 23). Themes that arose during data analysis that appear to match well with Agosto’s (2002) 
classification and application of Simon's theory, suggesting enrollment managers may utilize bounded rationality when making decisions.

\section{Time Constraints}

One of the most prominent and wide-spread themes among the participants was the feeling of not having enough time to accomplish their tasks. Whether it was adequate time to sufficiently think through a potential new recruitment initiative or effectively evaluate and measure operational processes, enrollment managers felt they were racing against time. Responses to the word association exercise yielded a consensus from the respondents that reflected this apparent battle against time, as if EMs were waging a battle against an enemy that was always gaining ground. Enrollment managers also expressed concerns about time constraints with their staff, who they felt were also overworked and "overused" for their particular expertise in a specific duty (such as data extraction and/or report generation).

Some enrollment managers extrapolated feelings of time limitations as being associated with their views of students' need for immediacy. One EM in particular shared her difficulty of trying to respond to student requests who expect a hastened response while trying also to provide accurate answers, claiming that quick and yet highly accurate answers were not always compatible. Another EM shared a situation where he had to "keep putting off" a project pertaining to out-of-state students, one that he deemed a fairly high priority, simply because there was no time to devote toward it. As that particular project was one that was mostly intrinsically motivated as opposed to an external instigator (such as his president or faculty who wanted their inquiries, requests, etc. handled first), it was relegated to a lower priority. These examples appear 
to demonstrate clear illustrations of Simon's bounded rationality approach in decisionmaking.

\section{Cognitive Constraints}

Another aspect of bounded rationality in Agosto's (2002) study was cognitive constraints of decision-making, also described as individuals who are feeling overloaded with possible options. While not as pronounced in the emergent themes, some examples were given by participants that may qualify under this characteristic. Some enrollment managers related their stories of how recruitment programs seem to keep being added without ever taking current endeavors away. Rather than taking time to assess and evaluate current recruitment events to determine their success in enticing new students to enroll, new ones seem to be added to their list of initiatives before EMs, who seem to have exhibited a desire for assessment and evaluation in overall enrollment management processes, have the opportunity to appraise existing plans.

Even day-to-day planning efforts became overwhelming for EMs to sift through in a sufficient, timely, and methodical manner as several of them shared in the theme of having to react versus actively plan. Some enrollment managers described having to "put out fires" as part of their daily routine while dealing with minor but equally draining minutia that comes from various sources. These may include walk-ins by staff or students, interruptions by dismayed parents wishing to argue an issue for their son or daughter, personnel disputes over seemingly trivial matters, and other unplanned disruptions that would force the EM into having to address whatever predicament may be presented them rather than carry out their pre-planned tasks.

Satisficing through Reduction of Choices 
The concept of satisficing is derived directly from bounded rationality as the individual settles on a less-than-optimal decision but one that could still meet basic expectations. Agosto (2002) relayed Simon's concept of satisficing through a "reduction" and eventual "termination" of options before accepting a possible solution (p. 23). Nearly all the EMs discussed the time limitations associated with decision-making, but some also voiced the desire to make the "right" decision. This implies that EMs recognized they may have reached a possible solution, but perhaps it was not the optimal one upon further reflection. Lack of time may have forced the reduction and led to the enrollment manager terminating the search for the ideal resolution in favor of an adequate one.

\section{Satisficing through Termination of Options}

Another enrollment manager relayed decisions she has made in the past where she "had" to make decisions based on a single fragment of information, whether it is based on an internal policy, perceived state or federal regulations, and the like just to achieve some type of resolution while knowing it was not thoroughly researched. She lamented the fact that some of these decisions led to negative impacts for other areas, and in retrospect, regretted she did not have the time to absorb the full gamut of potential outcomes. However, she also seemed to accept that these are the realities she faces in her role as enrollment manager of her institution.

In terms of the utilization of data and satisficing decision-making, enrollment managers conceded that the data elements used are imperfect, sometimes difficult to acquire, and simply not comprehensive enough to make exceptional decisions. These limitations have allowed the EM to be habitually content with the reality of using the 
incomplete data sets they have and caused them to replace gaps with experience-based intuition. As one EM stated, "Is it ideal? No way. Is it a reality? Yes." Examples such as these help illustrate the environment that surrounds enrollment managers as "complex' which seems to project conditions rife with opportunities for these individuals to satisfice as defined by Simon.

\section{Garbage Can Decision-Making}

Despite the possible insinuation that garbage can implies discarded ideas, this model actually reflects a mixture between problems, solutions, participants, and opportunities and has often been used to describe higher education organizational structures (Cohen, March, \& Olsen, 1972; Lane, 1980). The model suggests organizations function in a manner of organized anarchy which allows for a great deal of ambiguity when attempting to define organizational goals as well as who is going to accomplish them. Utilizing this theory has helped expound upon the complexities associated within a college or university as a large organization (Padgett, 1980).

Rowles' (2003) dissertation investigated the use of data in college and university decision-making practices and applied the garbage can theory in his analysis. Unlike satisficing, which assumes individuals are aware of an over-arching goal and choose an acceptable path of resolution, the garbage can theory suggests the environment itself is surrounded in goal ambiguity that leads individuals in the organization to rummage through the can for solutions to problems. Participants unload problems and solutions that have no linear, sequential flow into the symbolic garbage can. Instead, resolutions to problems may be resolved more through "temporal proximity" than a problem/solution 
relationship (p. 19). The following examples will be presented in a format to designate the non-linear flow and subsequent mixture of "problems," "solutions," and "people."

\section{Mixture of Problem, Sufficient Time, and Enrollment Managers}

EMs related a variety of situations where the decision-making process appeared to be fragmented and disjoined, much as the garbage can model suggests. One of the primary themes revealed through data analysis was how EMs felt they had no time to invest into a particular task, issue, problem, or idea. Instead, they could only afford to work on pieces of them at a time due to several interruptions (by staff, supervisor, students, or parents). Enrollment managers voiced frustration with the lack of ability to sequentially resolve an issue considering the fluidity of each day's schedule. While an EM may designate or block off time allocated in their day to develop strategies for an upcoming recruitment event, for example, his or her schedule would rarely allow this to occur chronologically. Some projects would have to be "shelved" or "put on a back burner" until a later time when an EM (or an employee designated by an EM) could devote effort toward addressing it adequately. By that time, the "problem" itself may have evolved or tangentially altered into a different direction requiring perhaps an adjusted solution.

Mixture of Enrollment Management Events, Data for Evaluation, and Enrollment Managers/Staff

A fairly substantial catalyst for an EM to feel empowered to make decisions is data, and several EMs bemoaned their struggles not only with acquiring data but also obtaining data they could "trust." Gathering and utilizing quality data not only enable EMs to measure the success of programs and initiatives they (or the institution) desired to 
continue, it also allowed them to show their supervisors endeavors that need to be ceased due to lack of productivity.

For example, one EM in particular shared her frustration over her feeling that their unit is always asked to take on new ideas or projects (mostly recruitment-related) while never taking away current programs. This stretches their already short-staffed workforce even further, and the EM voiced her regrets over not having the ability or the time to implement data measurement and evaluation for all programming that would showcase the productive as well as the inefficient ones. The "problem" of having desirable and effective recruitment programs yields "solutions" of adding additional quality events, programs, or projects. However, the "participant" (EM) does not have the ability to adequately evaluate the efficacy of each program and must pull out of the garbage can yet another overnight incoming freshman event targeting high academic standing students, for example, without truly knowing its success. Meanwhile, other events and programs still linger about in the can.

\section{Mixture of Multiple Problems, Reactive Solutions, and Problem-Solvers}

Similar to the theme associated with the lack of quality and timely data, enrollment managers often stated how they made decisions from a reactive versus proactive stance. Without knowing what projects net the most productive results, some EMs shared their perceptions of having to use a shotgun-like approach toward solving problems by throwing several possible solutions toward a problem to "see what sticks." This seems to line up well with the garbage can theory as multiple solutions are found in the can while the EM obtains several possible reactive solutions to potentially hurl them at a problem and hope one or more reach the target. Other EMs shared this line of 
thinking by echoing the lack of foresight when attempting to solve a problem. One enrollment manager in particular likened their enrollment management unit's ability to solve problems to running around trying to catch "fly balls" rather than being intentional with their efforts.

Although proclaiming decisions should be based upon data, many EMs commented how they relied on their experience or their "gut" to make decisions. While data provided direction and identified possible trends for success, some enrollment managers stated they would attempt to solve a problem by experience-driven past successes. Extrapolating further, another enrollment manager admitted to making decisions based on the culture of his institution as well as knowing what would be acceptable.

Additionally, when pressed for time or by conflicting sources of decision influencers (such as their president or faculty), enrollment managers seem to revert back to a guiding dictum of making decisions based "on the numbers." Realizing the importance of ensuring enrollment numbers and goals, EMs seemed to make decisions guided by the perception of what may make the greatest or most significant impact upon the number of enrolling students. These decision-making strategies appear to be yet another example of the garbage can model as the solutions may take many forms and are time-bound rather than routinely linear.

Mixture of Assigned Projects, Taking Immediate Action, and "Screamers"

On the subject of leadership and its impression upon decision-making in enrollment management, EMs shared numerous examples of how they directed their foci upon problems based on who is "screaming the loudest." Although an EM may be 
progressing through a specific problem with some diligence, the effort needed to desist upon the interjection by another influencer, such as the president, as the perception was that a president's needs are typically of higher priority. These perceived higher priority problems do not always emanate from the president, however, as several EMs voiced their appreciation and value for their staff's efforts to solve problems; therefore, they may cease their current project in favor of assisting their staff. Considering the varying roles of "participants" that may influence "problems" and "solutions," these prioritizations appear to be similar to a garbage can model.

Mixture of Consultant Recommendations, Selectively Choosing Solutions, and

\section{Enrollment Managers}

Relating to the notion of possible influencers upon decision-making, enrollment managers primarily voiced the lack of value associated with utilizing various enrollment management consulting services. Some EMs felt the extreme cost associated with these services did not provide enough return on investment, while others felt some recommendations may have merit. Most EMs who utilized consultants stated they might use particular strategies embedded within larger recommendations, as most felt those recommendation were not applicable to their specific institution. In a way, it seemed EMs used consultant-based solutions as if it were a buffet of choices rather than a strategic plan, thus further illustrating the mixture of problem and solution in the "can."

The garbage can model focused "less on the details of individual decision making and more on the aggregate flows of people, problems, and solutions through organizational networks" (Padgett, 1980, p. 583). Considering this model is utilized by organizations possessing "severe ambiguity" in their goals or objectives, their means to 
accomplish them, and who should be included or drive the decision-making process, it appears the enrollment management environment may possess enough complexity to fit this description.

\section{Conclusions}

When viewed in totality, the findings in this study point to specific conclusions concerning enrollment managers' decision-making practices. Enrollment managers work in a stressful, highly competitive and complex environment where expectations to meet enrollment goals govern their motives. Whether imposed upon them by their president, vice president, or a self-imposed mandate, the desire to attract and enroll new students remained a primary objective that rendered all other priorities to a secondary status.

With multiple interruptions into an EM's daily schedule, the ability for the enrollment manager to maintain consistent efforts on desired projects and plans was severely hindered. Enrollment managers in this study shared through multiple examples that they did not feel they had enough time to accomplish their tasks as thoroughly as they preferred and often either put projects on "back burners" or addressed the problem without full consideration of possible options. Describing daily interruptions as "minutia," enrollment managers acknowledged their time was stretched thin but also felt a duty to the students, parents, and staff who may be the source of the disruptions.

The manner in which EMs seem to make decisions appears to closely match the theory of bounded rationality proposed by Dr. Herbert Simon who suggested humans do not possess full access to the complexity of the environmental factors nor have a fully effective cognitive system that can maximize every real-life decision (Campitelli \& Gobet, 2010). The enrollment management environment itself exhibited characteristics 
suitable to allow EMs to make decisions in the context closely associated to the garbage can model. This was largely due to the ambiguity of goal setting and the mixture of participants, problems, and solutions in a figurative "can" of which temporal proximity appeared to be more of a factor in terms of what received attention first.

Although entrenched in their stance of valuing data in their decision-making, enrollment managers also used their own experience-driven intuition to help guide their decisions. Even when they were able to obtain the desired data, not all of them fully trusted it due to either an understanding of the technological system used to generate the data or because they were unable to acquire sufficient amounts and sources of data. Their ambivalent views toward data match the limitations enrollment managers have in terms of feeling confident and certain about their decisions as part of a larger strategic direction. When stable and effective leadership is absent, and EMs possess fragmented data sources, they are left to fill the gap with their instinctual awareness of enrollment management practice. In these cases, they seem fairly confident in their reliance on personal experience. Nevertheless, this reality forced enrollment managers to take action reactively instead of proactively. This ultimately left the EM feeling behind and anxious, almost as if s/he were in a constant chase where the prey remained just out of reach.

The concept and practice of enrollment management remains fluid and diverse in its application, and the evidence gathered in this study, particularly by the comments made by the EM participants, seem to suggest a preference toward this notion. As demonstrated by the EMs' comments concerning their apparent irritations toward consultants who claim individual application but seem to recommend tactics attempted and employed at many other institutions, a one-size-fits-all approach toward enrollment 
management decision-making is neither realistic nor preferred. The individuality of each institution, especially concerning its enrollment goals and objectives, appeared to be of high value by EMs in this study.

The competitive environment within enrollment management may also be predisposed toward having its leaders exhibit feelings of isolation. Considering many of the participants appeared to use the interview process itself as a means to express personal opinions, frustrations and passions - as if our interaction served as an outlet for them to articulate their perceptions-EMs may be internalizing stress associated with enrollment expectations and feel somewhat removed or in solitude compared to other administrative leaders across campus.

\section{Limitations}

Consistent with any study employing qualitative research methods, this study possessed certain limitations that should be revealed. Six specific enrollment managers and their institutions were selected due to their unique institutional culture as well as proximity in the Midwest region of the United States and cannot be assumed to accurately represent a nationwide population.

For the purposes of this study, only the enrollment management leader of the institution was selected for interviewing, which may have omitted other key voices. The documents and artifacts gathered were supplied either directly through the enrollment manager or their office, and the time and location(s) allowed for observations were dictated by each respective participant. Obtaining additional documentation as well as conducting observations at a specific time and/or location might have yielded additional perspectives. The total time spent on each campus varied, as did the opportunity to 
observe the interaction each EM had with his or her staff. The specific time of the year the visitations occurred also possibly limited or impacted the findings as the campuses were on a between-semester break, and the presence of students varied.

\section{Implications for Research, Theory, and Practice}

\section{Research}

This qualitative study investigated the decision-making practice of enrollment managers and may assist in expanding upon additional avenues concerning these subjects. In addition to the findings and recommendations found in Riley's (2007) and Rowles' (2003) dissertations concerning the examples of individuals in higher education institutions making less-than-ideal decisions, this study adds to the exploration of overall decision-making strategies by enrollment leaders.

Although many research-based sources offer descriptions and strategies associated with enrollment management, as well as decision-making approaches, few studies bring them both together, especially in a qualitative design. This study brings clarity to the decision-making realities experienced by EMs while trying to satisfy institutional enrollment expectations.

Using both of these models a priori proved worthwhile but in different contexts. Evidence found for bounded rationality and satisficing decision-making could be applied to the individual EM while the garbage can model reflected the organizational decisionmaking environment at large, thus providing a more encapsulating perspective.

\section{Theory}

The findings revealed in this study helped inform the decision-making environment of enrollment managers through the theoretical contexts of Simon's 
bounded rationality theory, as well as March, Cohen, and Olsen's garbage can model. The results seem to indicate that EM practitioners lacked the time and resources to evaluate all possible options (as well as their consequences) when attempting to resolve problems. This evidence seemed to reflect and build upon Simon's theories of bounded rationality and satisficing while adding further applicability to this primarily economicbased decision-making theory.

Heath and March (1994) discussed how the success and failure of a satisficing decision-making opportunity depended on the "richness of the search environment" (p.34), meaning those settings that have netted more successful decisions are more inclined to continue making decisions with positive results. Assessment of decisions made by EMs was not part of the scope of this study (only the recognition of satisficing decision-making), so utilization of bounded rationality theory may prove ineffective in this context without a method of evaluation.

Although the garbage can theory seemed to be a usable lens in terms of identification of this type of organizational anarchy applied in the context of the colleges and universities in this study, there may be limitations in its ability to invoke desired change. Choo (2006) asserts that this model, despite potential negative imagery based on its name, is not dysfunctional due to its ability to allow for decision-making to take place in organizations with ambiguously defined goals and decision-makers with limited time and resources. However, administrative leaders at campuses who recognize a garbage can model of decision-making at their campus and wish to change toward a model that may better carry out its strategic mission with more intentionality, this model seems to be lacking in terms of recommendations for change. 
The realities explained and revealed in this study also illuminate the theoretical perspective of constructionism as the data revealed the multiple realities experienced by enrollment managers. Considering the associated differences of each EM at their respective campuses (as well as how they seemed to prefer this individuality), this study brought to light how institutional culture influenced their perceptions, beliefs, and experiences.

\section{Practice}

The fluidity and varying practices of enrollment management suggest an unsteady environment that expects much out of the EMs asked to lead these units. Despite the numerous tools available to assist enrollment managers in their recruitment and retention of students, as well as the increased attention given to the practice of enrollment management by professional organizations and assorted affiliations, managing an institution's enrollment remains a complicated task. Examining the subjectivity and individuality of each EM to maximize enrollment opportunities while making decisions in a potentially complex environment was one of the main reasons for conducting this study. Enrollment managers who become familiar with the results of this study may be awakened to the realities impacting their ability to make quality, data-driven decisions as well as investigate opportunities to adjust the number of influencers and/or distractions on their ability to choose a more optimal solution to problems. Some specific recommendations for enrollment managers are listed below:

- Encourage the development of a realistic strategic enrollment plan - The few EMs in this study who felt as if their institution possessed stable leadership 
who developed an overarching strategic plan aligned with their strategic enrollment plan voiced more confidence in their ability to make decisions.

- Recognize data limitations - EMs in this study indicated a love/hate relationship with data for informing their decision-making. Whether the limits existed from lack of staff to create and gather the data or doubting the actual outputs, EMs should communicate these perceived inadequacies to their supervisors.

- Choose the data that matters - Contrary to the "if it moves, track it" mantra by national enrollment management practitioners, EMs may feel gain a sense of focus by prioritizing the data they believe to be the most vital to their decision-making. Agreeing upon the data sets or types will also prevent them from spending their already limited time measuring items that may not directly apply to their primary goals.

- Be comfortable with using experience to make decisions - EMs are expected to make data-driven decisions, but several enrollment managers in this study admitted to relying just as much on experience and intuition. Some proudly admitted their faith in this method while others volunteered it almost sheepishly. Considering the limitations surrounding timely, accurate, and quality data as reflected in this study, EMs should confidently utilize their instinct and self-awareness to bridge gaps in data.

- Structure the enrollment management unit to meet demands - This requires considerable financial and human resource support from the EM's supervisor, but the difficulty for an enrollment manager to accomplish even daily tasks 
was increased by being forced to respond to issues, interruptions, or other illtimed delays. As one EM stated, there can be very little "think" time as an enrollment manager when not sufficiently staffed or financially supported.

- Continue to advocate for your students and staff - EMs in this study projected a strong sense of pride in terms of protecting their institution's students as well as their own staff. Despite what recommendations may be offered by consultants that match apparent national best practices, EMs should champion the decisions and plans that best serve the profiles of their students while also providing supportive and stable environments for their staff who can often be overworked and under-appreciated.

The impact of the EMs' realities in this study to keep pursuing new students rather than switching to primarily retention-based initiatives may result in lower graduation rates as institutional resources continue to be devoted more toward attracting students rather than keeping and graduating the ones already enrolled. Although the EMs in this study acknowledged the importance of improving retention and graduation rates, it seemed as though they felt these concerns were secondary compared to the need for new student enrollment. This practice may have a long-lasting negative effect and resource drain as enrollment management units continue to focus upon new students rather than truly shaping and managing the institution's enrollment through a balance of recruiting, retaining, and graduating strategies.

Institutional presidents, executive boards, and other governing entities wishing to employ enrollment management strategies or alter existing ones may benefit from understanding and acknowledging the complexities associated with enrollment 
management units. They could plan, improve, or modify existing organizational formats to allocate more time and resources to empower the EM to make quality strategic decisions to reach enrollment goals for the institution.

\section{Recommendations for Future Research}

Enlarging not only the number of participants but also the geographical scope of the target institutions could greatly enhance and enrich our understanding of the experiences of enrollment managers. Interacting and meeting other key personnel involved with enrollment management decision-making, such as the president, respective vice-presidents, as well as staff or faculty who assist the EM could unearth additional data-rich analysis, while also providing additional voices with insight to the enrollment management environment.

Employing a decision-making instrument such as the 25 question General Decision-Making Survey established by Bruce and Scott (1995) could have helped in understanding each participant's general approach toward making decisions. Once known, tailored interview questions and overall observational strategies could be developed prior to an interview; this might shed additional perspective and applied meaning on each enrollment manager's environment.

Researchers wishing to pursue studies tangential to this one could do so in many paths. Some of these include a comparison of decision-making at public versus private institutions, or two-year colleges compared to four-year universities, or other comparison variations. Considering the varying organizational design of enrollment management units in this study, additional research could investigate the impact that organizational format may have upon decision-making practices. How would individual personality 
influence or impact the ability to make decisions? Are there personalities that may be perceived to more efficiently manage these areas than others? How can an enrollment manager's self-efficacy influence decision-making? What role does motivation play in enrollment management decision-making?

The experiences of enrollment managers in this study reflected a competitive environment with high expectations that impacted their decisions. It is unknown how much impact the pressure and realities documented by EMs may be specific to this particular environment or if these experiences may be reflected by most senior-level administrators in higher education. Individuals working in higher-level executive positions at colleges and universities may reveal similar perspectives to EMs in this study, but their perceptions, while outside the scope of this study, may be worthy of additional study.

Other facets of potential research may include technological influences as well as human or financial resources to obtain the type of quality data desired by EMs. Exploring the EM's insight as to the effectiveness of their decisions compared to their supervisor's or other staff's perspective might be investigated. The effect of a supportive faculty upon decision-making, the impact this competitive environment has upon the enrollment management leader, and the factors contributing to the perceptions of an effective campus-wide strategic plan or strategic enrollment plan are additional opportunities for examination. Researchers could also apply these inquiries toward other units within institutions of higher education other than enrollment management.

Researchers may wish to explore in greater detail specific decisions made by enrollment managers to potentially dissect their options and how they ultimately reached 
the solution. Rather than merely determining if a satisficing situation exists, researchers could investigate the process EMs undertook to recognize the opportunity to make a decision, process available options for benefits and consequences, and post-evaluation of the decision to determine if it were in fact an optimal or "good enough" choice as satisficing suggests (Bendor, 2003).

The garbage can model has been frequently applied to higher education settings, and the results from this study seem to suggest the existence of a garbage can model environment in the enrollment management context due to the complexity of participants, problems, and solutions. Further exploration into whether this model can be perceived as a positive or negative decision-making environment may be researched not only for the enrollment management unit itself but also its potential impact upon the institution at large.

Institutional presidents or the managerial committees or boards who oversee enrollment management units may shape their expectations for decision-making by their enrollment managers after further understanding of how these theories may exist (or be applied). It may need to be determined if the enrollment management environment itself is the component that tends to function in a garbage can model or if it is more a result of the functionality of the entire campus.

Additional implications for organizational theory such as the relationship between an enrollment management organizational culture and decision-making opportunities could be another potential avenue for further study. Viewing the results of this study through a social constructionism theoretical perspective may help explain how each participant discussed their decisions and the weight given to the impact upon "their" 
campus and what's best for "their students." These responses seemed to reflect how the EMs made meaning of their contextual decision-making efforts through their organizational culture.

\section{Final Summary and Reflection}

\section{Summary}

Enrollment managers are expected to make data-driven decisions in their efforts to recruit, retain, and graduate their students. The purpose of this study was to investigate the decision-making practices of enrollment managers with special attention devoted to their use of data within their processes. This concept was introduced in chapter 1 along with the statement of the problem, the purpose of the study, research questions guiding the study, a listing of definitions and terms used throughout the study, an introduction to discussing the theoretical framework, researcher statement to reflect interest and motivation for the study, an introduction to data collection and procedures, the significance of this particular study, and the potential impact upon research, theory, and practice.

To provide the reader with a foundational background in the related literature, chapter 2 presented sections on three main categories: an enrollment management overview, enrollment management in an organizational context, and decision-making strategies. Enrollment management sub-sections included the origins and growth of enrollment management, models of enrollment management units, expectations and characteristics found in EM leaders, the role of data for EMs, professional associations as well as consulting firms, and the future possibilities with enrollment management. Organizational theory components included examinations of culture, 
governance/leadership, planning and change, as an open system, and the infusion between power and politics. The chapter concluded with decision-making sub-sections of rationality, bounded rationality, and satisficing before leading to the garbage can model and bounded rationality viewed collectively.

Details concerning the study's design and methodology were presented in chapter

3. The guiding epistemology for this study was constructionism (sometimes described as social constructionism) due to the recognition of no true single reality but focused on multiple realities experienced and viewed by the individual who constructs his or her own meaning. The theoretical perspective utilized for this study was greatly influenced by Riley's (2007) study who also used Herbert Simon's theory of bounded rationality and satisficing as well as Cohen, March, and Olsen's (1972) garbage can model as a lens for viewing decision-making practices. Procedures and methods for collecting and analyzing the data were also outlined for this qualitative study before listing a trustworthiness table to confirm credibility, transferability, dependability, and confirmability of the study.

Chapter 4 presented detailed descriptions of site observations conducted at each participant's institution to help the reader provide a contextual foundation for presentation of the findings. A listing of participant demographics was also provided to offer additional background while revealing similarities and differences of EMs in this study.

The findings of the study were presented in chapter 5 as well as details related to collection procedures and coding methods to ensure the participants' voices were accurately represented. Themes that emerged during data review were unveiled, and a summation of the responses from the word association exercise was presented as well as 
its impact upon evaluation of the data. A listing and description of unexpected and absent themes was presented before concluding the chapter with a narrative portrait intended to capture the collective experiences and perceptions by the enrollment managers in this study.

Chapter six presented the discussion of findings after a brief summary followed by responses to the research questions. The discussion commenced through theory as the lens and their applicability to the findings. The study's conclusion was presented as well as associated limitations. Implications for research, theory, and practice were discussed before ending the chapter with recommendations for future research followed by a reflective summation.

\section{Personal Reflection}

Although I embarked on this study with the purpose and research questions primarily in mind, I experienced some degree of difficulty during the interview dialogues due to my desire to commiserate or relate my own experiences working as an administrator within an enrollment management unit. Several times during the interview I felt impulses to share in their responses as if to validate their experiences with my own. Admittedly, I did divert from the interview guide in the first couple of interviews by allowing some of the probing questions as well as my own interjections stray from the focus of the study. However, I do not believe this divergence detracted or weakened the richness of the responses - it may have in fact strengthened it by offering to the participants a shared experience of which they may have felt comforting.

The practice and philosophy of enrollment management has been and remains in flux. New or revised approaches toward recruiting, retaining, and graduating students 
seem to announced or presented through national associations, conferences, and consulting organizations at a hastening pace. Operational structure and format for enrollment management units vary and change seemingly as much as a new recruitment trend is proclaimed. Considering the debatable best practices that do not apply to every institution in a "one size fits all" approach as well as the fluctuating and potentially capricious institutional practice of enrollment management, how could one expect a decision-making effort by any means other than satisficing through a garbage can model?

It is my hope that enrollment management practitioners may in fact find some solace in their efforts to make diligently researched decisions (which often fall short of these expectations, as found in this study) by understanding there are additional managers who are faced with the same challenges to effective decision-making. I also realize enrollment managers are not the only ones to make satisficing decisions in complex environments, but institutional presidents, vice-presidents, and governing entities over enrollment planners should be mindful of these realities and limitations which may relieve some of the stress levels in this already high-pressure working environment with managers who let the worry and concern over meeting enrollment goals creep into their subconscious. 


\section{REFERENCES}

Agosto, D.E. (2002). Bounded rationality and satisficing in young people's web-based decision making. Journal of the American Society for Information Science and Technology, 53(1), 16-27.

Ahluwalia, P., Keil, M., Tiwana, A., \& Wang, J. (2007). The bounded rationality bias in managerial valuation of real options: Theory and evidence from it projects.

Decision Sciences, 38(1), 157-181.

American Association of Collegiate Registrars and Admissions Officers. (n.d.). About AACRAO. Retrieved June 25, 2012 from http://www.aacrao.org/AboutAACRAO.aspx

American College Testing. (n.d.). $28^{\text {th }}$ Annual act enrollment planners conference. Retrieved June 25, 2012 from http://www.act.org/epc/

Anderson, J. (2001). Obituary: Herbert A Simon (1916-2001). American Psychologist, 56(6-7), 516-518.

Anderson, D.K., Foley, C.J. \& Milner, B.J. (2008). From complex data to actionable information: Institutional research supporting enrollment management. New Directions for Institutional Research, 2008(137), 71-82.

Andreas, R.E., Krehbiel, L.E., Kuh, G.D., Lyons, J.W., MacKay, K.A., Schuh, J.H., . . Whitt, E.J. (1991). Involving colleges: Successful approaches to fostering student learning and development outside the classroom. San Francisco: Jossey-Bass.

Bachino, M. (2009). Two tongues for a dream: A hermeneutic study. (Doctoral dissertation, Duquesne University). Retrieved from 
http://search.proquest.com.argo.library.okstate.edu/docview/304882129/abstract/1 139DBDE0D7F4BD886/1? accountid=4117

Baldridge, J. V., Green, K.C., \& Kemerer, F. R. (1982). Strategies for effective enrollment management. Washington, D.C.: American Association of State Colleges and Universities.

Bendor, J. (2003). Herbert A. Simon: Political Scientist. Annual Review of Political Science, 6(1), p. 433-471.

Black, J. (1999). Navigating change in the new millennium: Enrollment leadership strategies. Washington, D.C.: American Association of Collegiate Registrars and Admissions Officers.

Black, J. (2004). Emerging Themes and Models. In Black (Ed.), Essentials of enrollment management: Cases in the field (pp. 1-14). Washington, D.C.: American Association of Collegiate Registrars and Admissions Officers.

Breen, R., Jenkins, A., \& Lindsay, R. (2002). Evidence-based management and action-ata-distance. Psychology Teaching Review, 10(1), 5-19.

Brown, R. (2004). Consideration of the origin of Herbert Simon's theory of "satisficing" (1933-1947). Management Decision, 42(10), 1240-1256.

Bruce, R.A., \& Scott, S.G. (1995). Decision-making style: The development and assessment of a new measure. Educational and Psychological Measurement, 55, 818-831.

Burke, W.W. (2007). Organization change: Theory and practice. Thousand Oaks, CA: Sage. 
Campitelli, G., \& Gobet, F. (2010). Herbert Simon's decision-making approach: Investigation of cognitive processes in experts. Review of General Psychology, 14(4), 354-364.

Carley, K. (1996). Organizational learning and personnel turnover. In M.D. Cohen \& L.S. Sproull, (Eds.), Organizational learning (pp.230-266). Thousand Oaks: Sage Publications.

Carpenter, M.H. \& Flowers, N. (2009). You don't have to be a statistician to use data: A process for data-based decision making in schools. Phi Delta Kappan, 91(2), 6467.

Choo, C.W. (2006). The knowing organization. New York: Oxford University Press.

Clagget, C.A. (1991). Institutional research: The key to successful enrollment management. Retrieved from http://www.eric.ed.gov/PDFS/ED332745.pdf

Clark, B.R. 1972). The organizational saga in higher education. Administrative Science Quarterly, 17(2), p. 178-184

Cohen, M. D., March, J. J., Olsen, J. P. (1972). A garbage can model of organizational choice. Administrative Science Quarterly, 17, 1-25.

College Board. (n.d.). Higher ed services. Retrieved from http://professionals.collegeboard.com/higher-ed

Cooley, V.E. \& Shen, J. (2008). Critical issues in using data for decision-making. International Journal of Leadership in Education, 11(3), p. 319-329.

Creswell, J. W. (2009). Research design. Los Angeles, CA: Sage.

Crotty, M. (2003). The foundations of social research. Thousand Oaks, Sage Publications. 
DeFeis, G.L. \& Rahman, N. (2009). Strategic decision-making: Models and methods in the face of complexity and time pressure. Journal of General Management, 35(2), p. 43-60.

Dolence, M. G., Lujan, H. D., \& Rowley, D. J (1997). Strategic change in colleges and universities: Planning to survive and prosper. San Francisco, CA: Jossey-Bass.

Dennis, M.J. (1998). A practical guide to enrollment and retention management in higher education. Westport, CN: Bergin \& Garvey.

Dixon, R.R. (1995). Enrollment Management in the Future. In Dixon (Ed.), Making Enrollment Management Work (pp. 89-98). San Francisco: Jossey-Bass.

Dixon, R.R. (1995). What is enrollment management? In Dixon (Ed.), Making Enrollment Management Work (pp. 5-10). San Francisco: Jossey-Bass.

Emerson, R. M., Fretz, R. I., \& Shaw, L. L. (1995). Writing ethnographic fieldnotes. Chicago, IL: University of Chicago Press.

Fioretti, G. \& Lomi, A. (2010). Passing the buck in the garbage can model of organizational choice. Computational \& Mathematical Organization Theory, 16(2), 113-143.

Guba, E. G., \& Lincoln, Y. S. (1985). Naturalistic Inquiry. Newbury Park, CA: Sage Publications

Gumport, P.J. (2001). Built to serve: The enduring legacy of public higher education. In P. Altbach, P. Gumport, \& B. Johnstone, (Eds.), In defense of the American university, (pp. 89-109), Baltimore: The John Hopkins University Press.

Heath, C. \& March, J.G. (1994). A primer on decision making: How decisions happen. New York: Maxwell Macmillan International 
Henden, E. (2007). Is genuine satisficing rational? Ethical Theory \& Moral Practice, 10(4), 339-352.

Hobsons. (n.d.). Higher education institutions. Retrieved from http://www.hobsons.com/education-professionals/higher-education/admissionsenrollment-professionals/

Hossler, D. (1984). Enrollment Management: An integrated approach. New York: College Entrance Examination Board.

Hossler, D. (1986). Creating effective enrollment management systems. New York: College Entrance Examination Board.

Hossler, D. (2004). How enrollment management has transformed - or ruined - higher education. Chronicle of Higher Education, 50(34), B3-B5.

Huddleston, Jr. T. (2000). Enrollment Management, In L. Johnsrud, \& V. Rosser, (Eds.), Understanding the work and career paths of mid-level administrators (pp.65-73). San Francisco: Jossey-Bass.

Jackall, R. (2010). Moral maxes: The world of corporate managers. Oxford: Oxford University Press.

Johnston, G.H. \& Kristovich, S.A.R. (2000). Community college alchemists: Turning data into information. New Directions For Community Colleges, (109), 63-73.

Keller, G. (2001). Governance the remarkable ambiguity. In P. Altbach, P. Gumport, \& B. Johnstone, (Eds.), In defense of the American university, (pp. 304-322), Baltimore: The John Hopkins University Press.

Kenny, J.D. (2009). Managing a modern university: Is it time for a rethink? Higher Education Research \& Development, 28(6), 629-642. 
Kerdeman, D. (2009). Why the best isn't so bad: Moderation and ideals in educational reform. Educational Theory, 59(5), 511-531.

Kuh, G.D., \& Whitt, E.J. (1988). The invisible tapestry: Culture in American colleges and universities. ASHE-ERIC Higher Education Reports, 17(1), 18-124.

Lane, J.E. (1980). Public administration and organizational development: Swedish higher education in the light of six theoretical models. European Journal of Education, 15(3), 299-311.

Layzell, D.T. (1997). The future is now: limitations of the crystal ball and other lessons learned. In Layzell (Ed.), Forecasting and Managing Enrollment and Revenue: An Overview of Current Trends, Issues, and Methods (pp 95-99). San Francisco: Jossey-Bass.

Lehman, D.R., Lyubomirsky, S., Monterosso, J., Schwartz, B., White, K., \& Ward, A. (2002). Maximizing versus satisficing: Happiness is a matter of choice. Journal of Personality \& Social Psychology, 83(5), 1178-1197.

Lovata, L. M. (1987). Behavioral theories relating to the design of information systems. MIS Quarterly, 11(2), 147-149.

Lucido, J.A. \& Schulz, A.A. (2011). Enrollment management, inc.: External influences on our practice. Retrieved from http://www.usc.edu/programs/cerpp/docs/EnrollmentManagementInc.ReportFIN AL_001.pdf

Lutz, F.W. (1982). Tightening up loose coupling in organizations of higher education. Administrative Science Quarterly, 27(4), 653-669. 
Machado-Taylor, M., Peterson, A., Taylor, J.S., \& Wilkinson, R.B. (2007). A practical guide to strategic enrollment management planning in higher education. Virginia Beach, VA: Educational Policy Institute. Retrieved from http://www.educationalpolicy.org/pdf/SEM\%20Guide.pdf

Major, C. \& Savin-Baden, M. (2010). Exploring the relevance of qualitative research synthesis to higher education research and practice. London Review of Education, $8(2), 127-140$.

March, J.G. (1991). How decisions happen in organizations. Human-Computer Interaction, 6, 95-117.

March, J.G. (1997). Understanding how decisions happen in organizations. In Shapira (Ed.), Organizational Decision Making (pp. 9-32). Cambridge, NY: Cambridge University Press.

March, J.G. and Simon, H.A. (1958). Organizations. New York: Wiley

Massa, R.J. (2004). Making Enrollment Management Work. In Black (Ed.), Essentials of enrollment management: Cases in the field (pp. 1-14). Washington, D.C.: American Association of Collegiate Registrars and Admissions Officers. Morgan, G. (2006). Images of organization. Newbury Park, CA: Sage.

Noel-Levitz. (n.d.). About noel-levitz. Retrieved from https://www.noellevitz.com/aboutnoel-levitz

Oklahoma State Regents for Higher Education, (2007). Policy and procedure manual. Retrieved from http://www.okhighered.org/state-system/policyprocedures/Chapter1-2007-Final.pdf 
Padgett, J.F. (1980). Managing garbage can hierarchies. Administrative Science Quarterly, 25(4), 583-604.

Patton, M. Q. (2002). Qualitative research and evaluation methods. Thousand Oaks, CA: Sage Publications.

Penn, G.L.D. (1999). Enrollment management for the $21^{\text {st }}$ century: Institutional goals, accountability, and fiscal responsibility. Washington, D.C.: George Washington University, Graduate School of Education and Human Development.

Poindexter, C.C. (2002). Meaning from methods: Re-presenting narratives of an hivaffected caregiver. Qualitative Social Work, 1(1), 59-76.

Presswood, K.R. (2011). Leadership attributes of enrollment managers in higher education institutions in the united states. (Doctoral dissertation, University of Florida). Retrieved from http://search.proquest.com.argo.library.okstate.edu/docview/883387063/fulltextP DF/13781CC6832555DF185/1 ?accountid $=4117$

Riley, T. (2007). Information, decision making and enrollment management in a public research university: A case study analysis using bounded rationality theory. (Doctoral dissertation, University of Pittsburgh). Retrieved from http://search.proquest.com.argo.library.okstate.edu/docview/304828040/fulltextP DF/13508C46AAA51498759/1? accountid=4117

Rowles, N.S. (2003). Halfway in the can: An examination of data use in college and university decision making. (Doctoral dissertation, University of Pennsylvania). Retrieved from 
http://search.proquest.com.argo.library.okstate.edu/docview/305306180/fulltextP DF/1392DEB07A11F118E08/1?accountid=4117

Salomonson, K. (2005). Assessment. In P. Bouman, \& C. Westman, (Eds.), Aacrao's basic guide to enrollment management (pp. 41-51). Washington, D.C.: American Association of Collegiate Registrars and Admission Officers.

Schumann, J.A. \& Streifer, P.A. (2005). Using data mining to identify actionable information: Breaking new ground in data-driven decision making. Journal of Education for Students Placed at Risk, 10(3), 281-293.

Simon, H. (1991). Bounded rationality and organizational learning. Organizational Science, 2(1), 125-134.

Simplicio, J.S.C. (2006). Shared governance: An analysis of power on the modern university campus from the perspective of an administrator. Education, 126(4), 763-768.

Stamats. (n.d.). About us. Retrieved from http://www.stamats.com/about/

T Culver. (2012, February 21). Re: Five data-informed steps to optimizing college student retention [Web log comment]. Retrieved from http://blog.noellevitz.com/2012/02/21/data-informed-steps-optimizing-collegestudent-retention/

T Culver. (2012, May 12). RE: Six strategies for collecting and using data in retention planning [Web log comment]. Retrieved from http://blog.noellevitz.com/2011/05/12/strategies-collecting-data-retentionplanning/ 
Tierney, W. G. (2008). The impact of culture on organizational decision-making: Theory and practice in higher education. Sterling, VA: Stylus Publishing.

Weaver, N.R. (2005). Presidential leadership: A phenomenological study of the leadership experience of oklahoma's regional university presidents. (Doctoral dissertation, University of Oklahoma). Retrieved from http://search.proquest.com.argo.library.okstate.edu/docview/305447165/previewP DF/13781C41CCC6D8A62B2/1 ?accountid=4117

Wolcott, H. F. (2009). Writing up qualitative research. Los Angeles, CA: Sage Publications.

Zey, M. (1992). Decision making: Alternatives to rational choice models. Newbury Park, CA: Sage Publications. 
APPENDIX A - Clagget's (1991, p. 2-3) Open-Ended Questions for EMs to Ask

- How widely known is the college? How do prospective students view the college? What other institutions are considered by prospective students?

- How can we increase the size of the applicant pool? How can we attract the students we would most like to enroll?

- How can we improve yield? How effective are our existing recruitment activities? What factors differentiate our college from its closest competitors and influence admitted students' final choices?

- What influence does financial aid have on student decisions to enroll and persist? What is the perceived campus culture or climate, and what influence does it have on retention and attrition?

- What proportion of a freshman class persists to graduation? Do any student subgroups exhibit significantly higher than average attrition? Why do some students persist while others do not?

- How successful are our alumni in their post-graduate endeavors? What proportion remain involved with the institution? What characteristics describe alumni donors? 
APPENDIX B - Suggested Research Reports (Dennis, 1998, p. 28-30)

Marketing and Enrollment

1. Demographic analysis of current and projected student markets

2. Geodemographic analysis of current and projected student markets

3. Enrollment forecasting

4. Monthly enrollment projection statistics for all student cohorts

5. New student enrollment projections

6. Comparison of projected and actual enrollments

7. Calculation of cost to recruit undergraduate and graduate students

8. Importance of factors in enrollment decision, including reputation, cost, class size, accessibility, faculty, and curriculum

9. Measurement of outreach activities for freshmen, transfer students, graduate students, adult learners, and corporate clients

10. Analysis of the effectiveness of direct mail program, college fairs, telecounseling, advertising, publications, and school visits

11. Enrollment Planning Service data analysis of the College Board

12. Admitted student questionnaires - undergraduate and graduate

13. Competitor analysis

14. Market share analysis

15. Comparative analysis of competitor institutions

16. Characteristics of students who enroll, including academic profile, income, gender, ethnicity, and geographic location

17. Characteristics of accepted students who did not enroll, including academic profile, income, gender, ethnicity, and geographic location

18. Orientation survey analysis

19. Survey by students of the admission, financial aid, registration, and bursar offices

20. Analysis of advertising, publications, and public relations campaigns

Financial Aid Analysis

1. Financial aid awards and enrollment yields

2. Admitted Student Questionnaire financial data analysis of the College Board including the percent of non-enrolling students not offered financial aid and the percent of non-enrolling students offered aid at competitor schools

3. Tuition pricing analysis

4. Income profile

5. Net tuition analysis

6. Tuition discounting and enrollment yields

7. Financial aid packaging analysis

8. Comparative tuition data

9. Part-time tuition pricing policies

10. Impact of tuition increases on enrollment and retention 


\section{Retention Reports}

1. Retention statistics from fall to spring semester

2. Retention statistics from spring to fall semester

3. Attrition and retention rates for freshmen, sophomores, juniors, seniors, minority students, adult students, part-time students, athletes, international students, and graduate students

4. Attrition vs. enrollment, fall to spring semesters and spring to fall semesters

5. Attrition rates by academic profile, income, gender, ethnicity, commuter or residential status, and year in school

6. Persistence rates by academic profile, income, gender, ethnicity, commuter or residential status, and year in school

7. Attrition rates by major and program

8. Persistence rates by major and program

9. Cooperative Institutional Research Program (CIRP) freshmen analysis

10. Attrition rates of adult learners

11. Persistence rates of adult learners

12. Withdrawal survey analysis

13. Leave of absence and probation analysis

14. Graduation statistics

15. High-risk student data analysis

Surveys and Publications

1. College Board Undergraduate and Graduate Surveys

2. Barron's Profiles

3. Peterson's Undergraduate and Graduate Surveys

4. Money magazine Survey

5. U.S. News \& World Report Survey

6. Orchard House Survey

Miscellaneous

1. Six-week survey of new freshmen and transfer students

2. Exit survey of graduating students

3. Economic impact of international students on the local economy

4. Alumni survey

5. Parent questionnaire

6. Publications focus-group analysis

7. Advertising focus-group analysis

8. Summer session course offering analysis

9. Salary statistics of graduates 
APPENDIX C - Professional Associations and Vendors

\section{Professional Associations}

After the Great Depression, the responsibilities of the admission officer began to blend with the responsibilities of the registrar by deemphasizing recruitment in favor of screening the desired applicants' academic credentials to determine whether the prospect matched up with the institution's student profile. The American Association of Collegiate Registrars and Admissions Officers (AACRAO) chose this blended approach (Hossler, 1984).

AACRAO's mission is “to serve and advance higher education by providing leadership in academic and enrollment services" (“About AACRAO,” n.d., para 2). Although this organization is classified as a nonprofit and voluntary association, institutions must pay annual membership fees to receive their benefits. AACRAO offers several conferences, webinars, and online courses for large fees including travel costs. Nearly 2,600 institutions from countries around the world currently claim membership to AACRAO.

Another not-for-profit organization, ACT, has mostly been known for their assessment test for students desiring entry into college. As the organization has grown, so has their offerings to institutions wishing to improve their enrollment efforts. Their annual Enrollment Planners Conference has been conducted for nearly 3 decades claiming their sessions present solutions to current issues including social media, technology, and the latest marketing and retention strategies. Conference goers will be extensively exposed to additional products and services during the conference, by many 
vendors in addition to ACT, so attendees will have further opportunities to spend valuable institutional dollars to address enrollment-based issues (" $27^{\text {th }}$ Annual," n.d.).

The College Board administers the nation's most widely used admission test, the SAT. Similar to ACT, the College Board has expanded their role into higher education by offering assistance with enrollment management. This organization offers a variety of tools and services intended to assist enrollment managers within the areas of recruitment and admissions, financial aid, advising and placement, among others. Similar to ACT, institutions may purchase lists of SAT test-takers for the purpose of targeted communication. This is one of the many products SAT offers colleges and universities (“Higher Ed Services," n.d.).

Hobsons proclaims itself to be a solutions-driven educational company and their products target the K-12 level as well as higher education. One of their premier products is their CRM (Client Relations Management system) that assures institutions who wish to maximize their recruitment efforts will see those expectations realized through this expensive tool. Their CRM solution requires a substantial financial commitment with yearly maintenance costs. Offering a variety of print and digital marketing solutions, Hobsons boasts effective data tracking methods for their communication efforts (“Hobsons," n.d.).

Noel-Levitz is another consulting firm specifically targeting higher education, and they claim their fundamental purpose is student success. Choosing to feature consulting services as their primary product, Noel Levitz expressly focuses on enrollment management related needs. Aside from assistance available in the areas of recruitment and financial aid, retention, enrollment and campus planning, marketing, web, and 
technologies, they offer services related to market research to help enrollment planners more effectively shape the student body. Hiring a Noel-Levitz consultant requires a strong financial commitment ("Noel-Levitz," n.d.).

Continuing with the focus of assisting higher education institutions with their marketing and recruitment plans, Stamats is a company that also offers a variety of staff to assist the enrollment planner, including researchers, strategists, consultants, writers, designers, and others. They offer similar services to the other consulting firms mentioned, but they claim to assist institutions to "break through the clutter" ("Stamats," n.d., para 3). 


\section{APPENDIX D1 - IRB Documents - Example Consent Form}

\section{Participant Consent Form}

Project Title: No Antidote for the Anecdote: The Decision-Making Strategies of Enrollment Management Professionals

Investigator: Adam Johnson, M.Ed., Doctoral Candidate, Oklahoma State University Kerri Kearney, MBA, Ed.D., Dissertation Advisor

Purpose: $\quad$ To reveal the environmental factors experienced by enrollment management professionals who are expected to make key, vital, datadriven decisions to meet their institution's enrollment goals. This is an empirical, qualitative research study that seeks the perspectives of enrollment managers, like you, who have lead your institution's enrollment management unit.

Procedures: Study participants will be asked to:

o complete an electronic demographic form

- complete the General Decision Making Style survey (25 questions) prior to site visit

○ participate in one $60+$ minute interview with the researcher

- participate in a word association exercise during the interview

$\circ$ participate with the researcher in a closing discussion at the end of the study

- allow researcher to observe the enrollment management facility to take notes

- allow researcher to sit in on a participant-led or participant-attended meeting of staff concerning enrollment management

Interactions and conversations with the researcher will be digitallyrecorded, and the researcher will keep hand-written notes as well.

Risks of Participation:

There are no known risks associated with this project which are greater than those ordinarily encountered in the traditional, workday, professional life.

Benefits: Despite the numerous tools available to assist enrollment planners in their recruitment and retention of students as well as the increased attention given to the practice of enrollment management by professional organizations and assorted affiliations, managing an institution's enrollment remains a complicated task. Gaining a greater understanding on how the decision-making progresses, and especially how well expectations match reality, will help improve the enrollment managers' ability to plan, improve, or modify existing decision-making practices. 
While no direct benefit exists for the interviewee compared to the expected results of this research, the interviewee will obtain satisfaction and knowledge of their contribution toward a greater understanding of the enrollment management decision-making environment.

Confidentiality:

The confidentiality of your participation in the study as well as all materials associated with your participation will be protected.

The records of this study will be kept private and participants will be identified by an assigned number only. Only the researcher will transcribe the recordings from the interviews. Any written results will discuss group findings and will not include information that will personally identify you. Research consent forms will be stored separately from raw data and in a file cabinet in the researcher's home. Raw data will be stored in the researcher's computer equipped with password-protection. Raw data may be kept up to 5 years. Only the researcher and individuals responsible for research oversight will have access to the records. It is possible that the consent process and data collection will be observed by university research oversight staff responsible for safeguarding the rights and wellbeing of people who participate in research.

Contacts: If you have any questions about this research, please contact Adam Johnson, University of Central Oklahoma, 100 N. University Drive, Edmond, OK 73034, 405-974-2385 or adjohnson@uco.edu, or Kerri Kearney, Oklahoma State University, kerr.kearney@okstate.edu.

If you have questions about your rights as a research volunteer, you may contact Dr. Shelia Kennison, IRB Chair, 219 Cordell North, Stillwater, OK 74078, 405-744-3377 or irb@okstate.edu.

Participant Rights:

Participation in this study is strictly voluntary and you may discontinue your related activities at any time without reprisal or penalty.

I have read and fully understand the consent form. I sign it freely and voluntarily. A copy of this form has been given to me.

$\overline{\text { Signature of Participant }} \quad \overline{\text { Printed Name }}$

I certify that I have personally explained this document before requesting that the participant sign it.

Signature of Researcher

Date 


\section{APPENDIX D2 - IRB Documents - Phone Solicitation}

Hello, this is Adam Johnson, and I am currently the Registrar at the University of Central Oklahoma as well as a doctoral candidate at Oklahoma State University. I am collecting data for research on the decision-making practices of enrollment management professionals. Do you have a few minutes to talk about it? (If yes, continue. If no, request follow-up time to talk).

I am targeting a total of six enrollment management leaders. I chose your institution due to its unique position and mission to serve students and am very interested in learning more about your role in the enrollment management area. Participation in this study would include a site visit by me.

Requested activities include a one-on-one interview, a tour of your enrollment management facilities, and observation, if possible, of a meeting that you may lead or in which you participate. The interview questions will consist of your experience with enrollment management and decision-making strategies and practices. The purpose of touring your enrollment management area is to observe and document the interaction between you and your staff as well as what information may be used for decision-making strategies. I may follow up with you over the course of my dissertation analysis (up to six months) for clarification. To protect your anonymity, all information will be kept strictly confidential and be kept in my possession.

If you are willing to participate, I will send you a consent form outlining further details about the study. Regardless of whether you participate, I greatly appreciate your time in considering this request.

Would you be interested in participating? (If yes, thank the participant and ask for site visit date. Remind them the consent form will be emailed within one day) 


\section{APPENDIX D3 - IRB Documents - Email Solicitation}

\section{Dear Colleague,}

My name is Adam Johnson, and I am currently the Associate Vice President/Registrar at the University of Central Oklahoma as well as a doctoral candidate at Oklahoma State University. I am collecting data for research on the decision-making practices of enrollment management professionals. I expect the results of this study will help contribute to our knowledge of enrollment managers' experiences in terms of attempting to make key, impactful, and data-driven decisions in an environment that may be dynamic and complex.

I am targeting a total of six enrollment management leaders. I chose your institution due to its unique position and mission to serve students and am very interested in learning more about your role in the enrollment management area. Participation in this study would include a site visit by me.

Requested activities are a one-on-one interview lasting approximately one hour, a tour of your enrollment management facilities, and observation, if possible, of a meeting that you may lead or in which you participate. The interview questions will consist of your experience with enrollment management and decision-making strategies and practices. The purpose of touring your enrollment management area is to observe and document the interaction between you and your staff as well as what information may be used for decision-making strategies. Outside from the on-site visit, I may follow up with you over the course of my dissertation analysis (up to six months) for clarification of data gathered as well as to answer any questions you may have. To protect your anonymity, all information will be kept strictly confidential and be kept in my possession.

If you are willing to participate, I will send you a consent form outlining further details about the study. Regardless of whether you participate, I greatly appreciate your time in considering this request.

You may reach me at adjohnson@uco.edu as well as by phone at (405) 974-2385. I look forward to hearing back from you soon.

- Adam Johnson 


\section{APPENDIX D4 - IRB Documents - Face-to-Face Solicitation}

\section{Dear Colleague,}

My name is Adam Johnson, and I am currently the Associate Vice President/Registrar at the University of Central Oklahoma as well as a doctoral candidate at Oklahoma State University. I am collecting data for research on the decision-making practices of enrollment management professionals. I expect the results of this study will help contribute to our knowledge of enrollment managers' experiences in terms of attempting to make key, impactful, and data-driven decisions in an environment that may be dynamic and complex.

I am targeting a total of six enrollment management leaders. I chose your institution due to its unique position and mission to serve students and am very interested in learning more about your role in the enrollment management area. Participation in this study would include a site visit by me.

Requested activities are a one-on-one interview lasting approximately one hour, a tour of your enrollment management facilities, and observation, if possible, of a meeting that you may lead or in which you participate. The interview questions will consist of your experience with enrollment management and decision-making strategies and practices. The purpose of touring your enrollment management area is to observe and document the interaction between you and your staff as well as what information may be used for decision-making strategies. Outside from the on-site visit, I may follow up with you over the course of my dissertation analysis (up to six months) for clarification of data gathered as well as to answer any questions you may have. To protect your anonymity, all information will be kept strictly confidential and be kept in my possession.

If you are willing to participate, I will send you a consent form outlining further details about the study. Regardless of whether you participate, I greatly appreciate your time in considering this request.

You may reach me at adjohnson@uco.edu as well as by phone at (405) 974-2385. I look forward to hearing back from you soon.

Sincerely,

Adam Johnson, Doctoral Candidate

Oklahoma State University 
APPENDIX D5 - IRB Documents - Participant Basic Demographic Form

1. Please circle appropriate description: male female

2. Degrees you hold and the years received:

3. Current job title:

4. Number of years at current position:

5. Title of your direct supervisor:

6. Other jobs held at current institution including years of service (e.g. Assistant Director, Financial Aid, 5 years):

7. Number of people who directly report to you and their titles (A departmental organizational chart may be provided in lieu of a response to this question):

8. Your professional memberships (e.g. OACRAO, SACRAO, AACRAO, OACADA, etc.):

9. Subscription services about enrollment management or higher education in general (e.g. Chronicle of Higher Ed; University Business; Magna's Recruitment $\&$ Retention newsletter, etc.) that you currently read:

10. List any technological tools you use to aid in enrollment management planning or decision-making (e.g. a CRM system)

11. Please describe anything else you personally use to guide your enrollment management decision-making: 


\section{APPENDIX D6 - IRB Documents - Approval Letter}

\section{Oklahoma State University Institutional Review Board}

Date:

Thursday, November 29, 2012

IRB Application No

Proposal Title:

ED12188

No Antidote for the Anecdote: The Decision-Making Strategies of Enrollment Management Professionals

Reviewed and

Exempt

Processed as:

Status Recommended by Reviewer(s): Approved Protocol Expires: 11/28/2013

Principal

Investigator(s):

Adam W. Johnson

4720 Deer Ridge Blvd

Kerri Shutz Kearney

Yukon, OK 73099

315 Willard

Stillwater, OK 74078

The IRB application referenced above has been approved. It is the judgment of the reviewers that the rights and welfare of individuals who may be asked to participate in this study will be respected, and that the research will be conducted in a manner consistent with the IRB requirements as outlined in section 45 CFR 46 .

Q The final versions of any printed recruitment, consent and assent documents bearing the IRB approval stamp are allached to this letter. These are the versions that must be used during the study.

As Principal investigator, it is your responsibility to do the following:

1. Conduct this study exactly as it has been approved. Any modifications to the research protocol must be submitted with the appropriate signatures for IRB approval. Protocol modificalions requiring approval may include changes to the litle. $\mathrm{PI}_{i}$ advisor, funding status or sponsor, subjecl population composition or size, recruitment, inclusionfexclusion criteria, research site, research procedures and composition or size, recruitment,

2. Submit a request for continuation if the sludy extends beyond the approval period of one calendar

year. This continuation must receive IRB review and approval before the research can continue.

3. Reporl any adverse events to the IRB Chair promptly. Adverse events are those which are

unanticipated and impact the subjects during the course of this research; and

4. Notify the IRB office in writing when your research project is complete.

Please note that approved protocols are subject to monitoring by the IRB and that the IRB office has the aulhority to inspect research recoros associated with this prolocol at any time. If you have questions about the aulhority to inspect research records associated with this prolocol al any time. If you have questions about the (phone: 405-744-5700. dawnett.watkins@okstate.edu).

i. Sincerely,

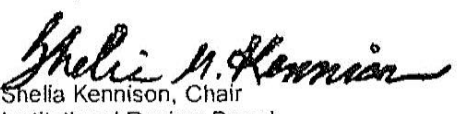

Institutional Review Board 


\section{APPENDIX E - Interview Guide Questions}

Tell me about yourself.

What is a typical day like in your position?

What is your understanding of the practice of enrollment management?

Please describe for me how enrollment management is conducted on your campus.

How do you feel about your enrollment management efforts at your campus?

What kinds of things do you do to become a better enrollment manager?

How do you make decisions?

What things should you consider when making decisions impacting enrollment management?

Do you consider those things when making decisions?

How do you prioritize things that may influence your decision?

Does anyone help influence your decision?

What data should you use in making decisions?

How do you use data in your decision-making?

Do you now, or have you ever consulted with an outside firm or service to assist your decision-making?

Please describe a situation when a decision you made was successful.

Why do you feel it was a successful decision?

Please describe an example of a poor decision you made.

Why do you feel it was a poor decision?

Overall, how successful or effective do you feel you are at making decisions?

If you could go back and give yourself advice when you first started in enrollment management, what would you say? 


\section{APPENDIX F - Word Association Exercise}

Participant:

1. Enrollment management

2. Decision-making

3. Campus-wide teams

4. Recruitment

5. Retention

6. Graduation

7. Data

8. Resources

9. Time

10. Enrollment expectations 
VITA

Adam W. Johnson

Candidate for the Degree of

Doctor of Education

Thesis: BALANCING DATA, TIME, AND EXPECTATIONS: THE COMPLEX

DECISION-MAKING ENVIRONMENT OF ENROLLMENT

MANAGEMENT

Major Field: Higher Education

Biographical:

Education:

Completed the requirements for the Doctor of Education in Higher Education at Oklahoma State University, Stillwater, Oklahoma in July, 2013.

Completed the requirements for the Master of Science in Education at The University of Central Oklahoma, Edmond, OK/United States of America in 2005.

Completed the requirements for the Bachelor of Arts in English at The University of Central Oklahoma, Edmond, OK/United States of America in 1995.

Experience:

Associate Vice-President/Registrar, The University of Central Oklahoma, 2010 - present

Associate Registrar, The University of Central Oklahoma, 2009 - 2010

Director, Prospective Student Services/Scholarships, The University of Central Oklahoma, 2005 - 2009

Professional Memberships: American Association of Collegiate Registrars and Admission Officers (AACRAO), Oklahoma Association of Collegiate Registrars and Admissions Officers (OACRAO), Oklahoma College Day/Night Coordination Committee (OCD/NCC) 